\title{
Über die allgemeinste Form der Gesetze der chemischen Kinetik homogener Systeme
}

von

\author{
Rud. Wegscheider.
}

Aus dem I. chemischen Laboratorium der k. k. Universität in Wien.

(Mit 1 Tafel.)

(Vorgelegt in der Sitzung am 15. Juni 1900.)

Die Aufstellung der Gleichungen für die Reactionsgeschwindigkeiten ist in der Regel eine sehr einfache Sache, wenn die stattfindenden Reactionen bekannt sind. Die rechnerischen Schwierigkeiten fangen auch in complicierteren Fällen erst mit der Integration der Differentialgleichungen an.

Nichtsdestoweniger kann unter Umständen ein Schema für die Geschwindigkeitsgleichungen nützlich werden, welches alle Arten von Reactionen (Neben-, Gegen-, Folgewirkungen, katalytische Wirkungen) umfasst. Ein solches Schema kann insbesondere zur Beantwortung der Frage, welche Arten von Reactionsgleichungen mit einem bestimmten, experimentell gefundenen Reactionsablaufe verträglich sind, nützlich sein.

\section{Formulierung der Reactionsgeschwindigkeiten in beliebigen homogenen Systemen.}

§. 1. Die allgemeinste Form der in einem System möglichen Reactionsgleichungen erhält man offenbar in folgender Weise. Es seien die chemischen Formeln der in dem System $\mathrm{zu}$ irgendeiner $Z$ eit vorhandenen Molekelarten $M_{1}, M_{2}, M_{3}$ u. s.w. Dann ist jede in dem System mögliche Reaction darstellbar durch eine Reactionsgleichung von der Form $n_{1} M_{1}+n_{2} M_{2}+\ldots$ $\rightarrow a_{1} M_{1}+a_{2} M_{2}+\ldots$, in welcher $n_{1}, n_{2} \ldots$ die Anzahlen der bei 
der Reaction verschwindenden, $\alpha_{1}, \alpha_{2} \ldots$ die Anzahlen der bei der Reaction entstehenden Molekeln bedeuten. Nullwerden der betreffenden $n$ oder $\alpha$ bedeutet, dass die Molekelart auf der betreffenden Seite der Reactionsgleichung nicht vorkommt. Ist für denselben Stoff $n$ und $\alpha$ von Null verschieden, so handelt es sich um katalytische Wirkungen, wie später noch näher auseinandergesetzt wird.

Die Coefficienten $n_{1}, n_{2} \ldots, \alpha_{1}, \alpha_{2} \ldots$ sind durch Beziehungen miteinander verknüpft, welche die Erhaltung der Masse jedes einzelnen Grundstoffes ausdrücken. Diese Beziehungen sind leicht zu formulieren. Ich gehe darauf nicht ein, da sie im folgenden nicht zur Verwendung gelangen.

Befinden sich unter den Molekelarten Ionen, so müssen sich im System jederzeit gleich viele positive und negative Ladungen befinden. Daraus folgt, dass die Differen $z z w$ is chen der Zahl der positiven und negativen Ladungen auf beiden Seiten der Reactionsgleichung gleich sein muss. Hiedurch ist ebenfalls eine Beziehung zwischen den Coefficienten $n$ und a gegeben.

Finden in einem System mehrere Reactionen statt, so hat man die Reactionsgleichungen

$$
\left.\begin{array}{rc}
\text { I. } & n_{1} M_{1}+n_{2} M_{2}+\ldots \rightarrow \alpha_{1} M_{1}+\alpha_{2} M_{2}+\ldots \\
\text { II. } & o_{1} M_{1}+o_{2} M_{2}+\ldots \rightarrow \beta_{1} M_{1}+\beta_{2} M_{2}+\ldots \\
\text { III. } & p_{1} M_{1}+p_{2} M_{2}+\ldots \rightarrow \gamma_{1} M_{1}+\gamma_{2} M_{2}+\ldots \\
\text {. } & \ldots \ldots \ldots \ldots \ldots \ldots
\end{array}\right\} .
$$

§. 2. Im folgenden wird die Voraussetzung gemacht, dass das Volum des Systems während der Reaction unverändert bleibt, eine Voraussetzung, die bisher in der chemischen Kinetik immer ausdrücklich ${ }^{1}$ oder stillschweigend gemacht worden ist.

§. 3. Die Geschwindigkeiten der einzelnen Reactionen drückt man am besten in der Art aus, wie ich es bereits in einer früheren Abhandlung ${ }^{2}$ gethan habe. Die zwei Seiten

I Siehe z. B. Ostwald, Lehrb. der allg. Chemie, 2. Aufl., II, 2, S. 201 (1896).

2 Z. f. physikal. Chemie, 30, 594 (1899). 
einer Reactionsgleichung stellen zwei Formen einer hylotropen Gruppe ${ }^{1}$ dar. Führt man jede solche hylotrope Form wie eine Molekel in die Rechnung ein, so kann man den Concentrationszuwachs, welchen der auf der rechten Seite der Reactionsgleichung I stehende Complex seit Beginn der Zeitzählung (also während der Zeit $t$ ) erfahren hat, mit $x$ bezeichnen. Mit anderen Worten: Wird der auf der rechten Seite der Reactionsgleichung I stehende Complex $\alpha_{1} M_{1}+\alpha_{2} M_{2}+\ldots$ als eine Molekel betrachtet, so gibt $x$ die Anzahl dieser Molekeln in der Volumeinheit an, welche seit Beginn der Zeitzählung gebildet worden sind. $\frac{d x}{d t}$ gibt dann die Geschwindigkeit der Reaction I an, und zwar ebensowohl den Zuwachs der auf der rechten Seite 'der Reactionsgleichung stehenden hylotropen Form, wie die Abnahme der auf der linken Seite der Reactionsgleichung stehenden Form.

Analog kann man die Geschwindigkeit der Reaction II mit $\frac{d y}{d t}$ bezeichnen, wo $y$ die in Molekeln ausgedrückte Menge des seit Beginn der Zeitzählung in der Raumeinheit gebildeten Complexes $\beta_{1} M_{1}+\beta_{2} M_{2}+\ldots$ bedeutet, wenn dieser Complex als ein e Molekel gerechnet wird.

Die analogen Veränderlichen für die Reactionen III u. s. w. sollen mit $z$ u. s. w. bezeichnet werden.

§. 4. Mit den so definierten Veränderlichen $x, y, z \ldots$ sind die zur Zeit $t$ eingetretenen Concentrationsänderungen der Molekelarten $M_{1}, M_{2} \ldots$ durch stöchiometrische Beziehungen verknüpft, welche unmittelbar aus den Reactionsgleichungen folgen.

Bezeichnet $\xi_{1}$ die zur Zeit $t$ eingetretene Abnahme der Concentration der Molekelart $M_{1}$ gegenüber der Anfangsconcentration $(t=0)$, so ist

$$
\xi_{1}=\left(n_{1}-\alpha_{1}\right) x+\left(o_{1}-\beta_{1}\right) y+\left(p_{1}-r_{1}\right) z+\ldots
$$

Denn wenn die nach der Reactionsgleichung I umgesetzte Menge $x$ beträgt, so sind $n_{1} x$ Molekeln von $M_{1}, n_{2} x$ Molekeln

1 Ostwald, Lehrb. der allg. Chemie, 2. Aufl., II, 2, S. 298 (1897). 
von $M_{2}$ u. s. w. in der Raumeinheit verschwunden und dafür $\alpha_{1} x$ Molekeln von $M_{1}, \alpha_{2} x$ Molekeln von $M_{2}$ u. s. w. gebildet worden.

Ebenso sind $o_{1} y$ Molekeln von $M_{1}, o_{2} y$ Molekeln von $M_{2}$ u. s. w. in der Raumeinheit verschwunden und $\beta_{1} y$ Molekeln von $M_{1}, \beta_{2} y$ Molekeln von $M_{2}$ u. s. w. gebildet worden, wenn der eingetretene Umsatz nach der Reactionsgleichung II den Betrag $y$ erreicht hat.

Die Summierung der bei den einzelnen Reactionen eingetretenen Concentrationsabnahmen von $M_{1}$ gibt den obigen Ausdruck für $\xi_{1}$.

Bezeichnen ferner $\xi_{2}, \xi_{3} \ldots$ die Abnahmen der Concentrationen der Molekelarten $M_{2}, M_{3} \ldots$, so erhält man die Gleichungen

$$
\left.\begin{array}{l}
\xi_{1}=\left(n_{1}-\alpha_{1}\right) x+\left(o_{1}-\beta_{1}\right) y+\left(p_{1}-\gamma_{1}\right) z+\ldots \\
\xi_{2}=\left(n_{2}-\alpha_{2}\right) x+\left(o_{2}-\beta_{2}\right) y+\left(p_{2}-\gamma_{2}\right) z+\ldots \\
\xi_{3}=\left(n_{3}-\alpha_{3}\right) x+\left(o_{3}-\beta_{3}\right) y+\left(p_{3}-\gamma_{3}\right) z+\ldots \\
\ldots \ldots \ldots \ldots \ldots \ldots \ldots \ldots \ldots \ldots \ldots \ldots \ldots \ldots \ldots \ldots \ldots \ldots \ldots
\end{array}\right\} .
$$

§. 5. Bei Gegenwart von Elektrolyten oder allgemeiner von hylotropen Formen, zwischen denen sich mit sehr großer Geschwindigkeit ein Gleichgewicht herstellt, sind in manchen Fällen nicht die Änderungen der Concentrationen der einzelnen Molekelarten von Interesse, sondern die Änderungen der Concentration der gesammten hylotropen Gruppe. Wenn z. B. Salzsäure an einer Reaction betheiligt ist, werden in vielen Fällen nicht die Concentrationsänderungen derWasserstoff-oder Chlorionen oder der undissociierten Chlorwasserstoffmolekeln von Interesse sein, sondern die Änderung der Gesammtconcentration der Salzsäure.

In solchen Fällen kann folgender Satz angewendet werden: Für die Änderung der Gesammtconcentration einer hylotropen Gruppe sind die zwischen den Molekelarten dieser Gruppe auftretenden Reactionen mit Gegenwirkungen ohne Einfluss.

Wenn die Stoffe $M_{1}, M_{2} \ldots M_{m}, M_{m+1} \ldots M_{m+12}$ einer hylotropen Gruppe angehören, derart, dass zwischen ihnen die Reactionen stattfinden 
$\left.\nu_{1} M_{1}+\nu_{2} M_{2}+\ldots+\nu_{m} M_{m} \rightarrow \nu_{m+1} M_{m+1}+\ldots+\nu_{m+n} M_{m+n}\right\rangle$

und

$\nu_{n+1} M_{m+1}+\ldots+\nu_{m+n} M_{m+n} \rightarrow \nu_{1} M_{1}+\nu_{2} M_{2}+\ldots+\nu_{m} M_{n n}$,

und wenn die nach der ersten Gleichung umgesetzte Menge $x$, die nach der zweiten Gleichung umgesetzte Menge $y$ ist, so ist jener Theil von $\xi_{1}$, welcher auf diese beiden Reactionen zurückzuführen ist, gegeben durch $v_{1} x-v_{1} y=v_{1}(x-y)$, der entsprechende Antheil von $\xi_{m+1}$ durch $y_{m+1}(y-x)$.

Verlaufen daneben noch andere Reactionen, so nimmt $\xi_{1}$ die Form an

$$
\xi_{1}=\nu_{1}(x-y)+\left(p_{1}-\gamma_{1}\right) z+\ldots
$$

Ähnlich sind die Ausdrücke für die anderen $\xi$.

Bezeichnet man die Concentrationsabnahme des wie eine Molekel in Rechnung gezogenen Complexes $\nu_{1} M_{1}+\nu_{2} M_{2}+\ldots$ $\ldots+v_{m} M_{m}$ (A) mit $\zeta_{1}$, die Concentrationsabnahme des Complexes $\gamma_{m+1} M_{m+1}+\ldots+\nu_{n+n} M_{m+n}$ (B) mit $\zeta_{2}$, so ist die gesammte Concentrationsabnahme der hylotropen Form $\zeta_{1}+\zeta_{2}$. Es seien nun $M_{1}$ und $M_{n+1}$ zwei Molekelarten, deren Concentrationen als Maß für die Concentrationen der Complexe $A$ und $B$ dienen können. (Hiezu eignet sich nicht jede zur hylotropen Form gehörige Molekelart. Ist z. B. Salzsäure neben anderen Säuren vorhanden, so geben die Concentrationen der Molekelarten $\overline{\mathrm{C}}$ l und $\mathrm{HCl}$ zugleich die Concentrationen der Complexe $\stackrel{+}{\mathrm{H}}+\overline{\mathrm{C}}$ lnd $\mathrm{HCl}$ an. Dagegen sind in diesem Falle die Wasserstoffionen nicht zur Bestimmung der Concentration der hylotropen Gruppe der Salzsäure verwendbar.)

Dann ist $\zeta_{1}=\frac{1}{\nu_{1}} \xi_{1}$ und $\zeta_{2}=\frac{1}{\nu_{m+1}}-\xi_{m+1}$. Daher

$$
\begin{aligned}
\zeta_{1}+\zeta_{2}=(x-y)+\frac{\left(p_{1}-\gamma_{1}\right) z+\ldots}{\gamma_{1}}+ & y-z)+ \\
& +\frac{\left(p_{m+1}-\gamma_{m+1}\right) z+\ldots}{\gamma_{m+1}} .
\end{aligned}
$$

Die auf die Reactionen mit Gegenwirkungen bezüglichen Veränderlichen $x$ und $y$ fallen heraus. 
Handelt es sich also um die Concentrationsänderungen der gesammten hylotropen Form, so können die zwischen den Molekelarten der hylotropen Form stattfindenden, zu Gleichgewichten führenden Reactionen mit Gegenwirkungen unberücksichtigt bleiben.

Für $\mathrm{H}_{2} \mathrm{CO}_{3}$ hat man $z$. B. die Gleichgewichte

$$
\mathrm{H}_{2} \mathrm{CO}_{3} \rightleftarrows \mathrm{HCO}_{3}+\stackrel{+}{\mathrm{H}} \text { und } \overline{\mathrm{HCO}}_{3} \rightleftarrows \overline{\mathrm{CO}}_{3}+\stackrel{+}{\mathrm{H}} \text {. }
$$

Die Veränderlichen dieser vier Reactionen seien $x, y, z, u$. Daneben sollen noch andere Reactionen stattfinden, bei denen einzelne der in den Gleichgewichten vorkommenden Molekelarten gebildet oder verbraucht werden und deren Veränderliche $v, w$. . seien.

Wird $\mathrm{H}_{2} \mathrm{CO}_{3}$ als $M_{1}, \mathrm{H} \overline{\mathrm{C}} \mathrm{O}_{3}$ als $M_{2}, \overline{\mathrm{C}} \overline{\mathrm{O}}_{3}$ als $M_{3}$ bezeichnet, so ist

$$
\begin{aligned}
& \xi_{1}=x-y+\left(r_{1}-\varepsilon_{1}\right) v+\left(s_{1}-\eta_{1}\right) w+\ldots \\
& \xi_{2}=-x+y+z-u+\left(r_{2}-\varepsilon_{2}\right) v+\left(s_{2}-\eta_{2}\right) w+\ldots \\
& \xi_{3}=-z+u+\left(r_{3}-\varepsilon_{3}\right) v+\left(s_{3}-\eta_{3}\right) w+\ldots
\end{aligned}
$$

Die Gesammtänderung der Concentration der Kohlensäure wird gegeben durch

$\xi_{1}+\xi_{2}+\xi_{3}=$ $=\left(r_{1}+r_{2}+r_{3}-\varepsilon_{1}-\varepsilon_{2}-\varepsilon_{3}\right) v+\left(s_{1}+s_{2}+s_{3}-\eta_{1}-\eta_{2}-\eta_{3}\right) w+\ldots$

§. 6. Bezeichnen $A_{1}, A_{2} \ldots$ die Anfangsconcentrationen der Molekelarten $M_{1}, M_{2}$.., so sind die Geschwindigkeitsgleichungen

$$
\left.\begin{array}{l}
\frac{d x}{d t}=k_{1}\left(A_{1}-\xi_{1}\right)^{n_{1}}\left(A_{2}-\xi_{2}\right)^{n_{2}} \ldots \\
\frac{d y}{d t}=k_{2}\left(A_{1}-\xi_{1}\right)^{o_{1}}\left(A_{2}-\xi_{2}\right)^{o_{2}} \ldots \\
\ldots \ldots \ldots \ldots \ldots \ldots \ldots \ldots \ldots \ldots \ldots \ldots
\end{array}\right\}
$$

in welchen dann die $\xi$ durch $x, y \ldots$ ausgedrückt werden können. $k_{1}, k_{2} \ldots$ sind die Geschwindigkeitscoefficienten der einzelnen Reactionen.

§. 6 a. Befinden sich unter den Molekelarten, welche in nine Geschwindigkeitsgleichung eingehen, solche, welche mit 
anderen Molekelarten durch ein sich unendlich rasch einstellendes Gleichgewicht verbunden sind, so ist die Gleichgewichtsbedingung in jedem Augenblicke erfüllt.

Es sei diese Gleichgewichtsbedingung

$$
\left(A_{1}-\xi_{1}\right)^{n_{1}}\left(A_{2}-\xi_{2}\right)^{n_{2}} \ldots=x\left(A_{\nu+1}-\xi_{\nu+1}\right)^{n_{\nu}+1}\left(A_{\nu+2}-\xi_{\nu}+2\right)^{n_{\nu}}+2 \ldots,
$$

wo $x$ die Gleichgewichtsconstante ist.

Dann ist sofort ersichtlich, dass die Geschwindigkeitsgleichungen

$$
\frac{d x}{d t}=k_{1}\left(A_{1}-\xi_{1}\right)^{n_{1}}\left(A_{2}-\xi_{2}\right)^{n_{2}} \ldots\left(A_{e}-\xi_{e}\right)^{n_{e}}\left(A_{f}-\xi_{f}\right)^{n_{f}} \ldots
$$

und

$$
\frac{d x}{d t}=\dot{k}_{1} \varkappa\left(A_{\nu+1}-\xi_{\nu}+1\right)^{n_{\nu}+1}\left(A_{\nu+2}-\xi_{\nu}+2\right)^{n_{\nu}+2} \ldots\left(A_{e}-\xi_{e}\right)^{n_{e}}\left(A-\xi_{f}\right)^{n_{f}} .
$$

identisch sind. $M_{c}, M_{f} \ldots$ sollen Molekelarten sein, die nicht am Gleichgewichte betheiligt sind.

In solchen Fällen ist es daher nicht möglich, zu entscheiden, ob die Reaction nach der Gleichung

$$
n_{1} M_{1}+n_{2} M_{2}+\ldots+n_{e} M_{c}+n_{f} M_{f}+\ldots \rightarrow X
$$

oder nach der Gleichung

$$
n_{\nu+1} M_{\nu+1}+n_{\nu+2} M_{\nu+2}+\ldots+n_{e} M_{e}+n_{f} M_{f}+\ldots \rightarrow X
$$

vor sich geht. Nur erhält man verschiedene Zahlenwerte der Constanten, je nachdem man der Rechnung die eine oder die andere Reactionsgleichung zugrunde legt.

Von praktischer Wichtigkeit ist dieser Fall bei Reactionen, an welchen Elektrolyte betheiligt sind. Es lässt sich demgemäß aus Geschwindigkeitsbeobachtungen nicht entscheiden ob Elektrolyte als undissociierte Molekeln oder in Form der sämmtlichen aus den undissociierten Molekeln entstehenden Ionen auftreten (z. B. ob in einer Reactionsgleichung $n H X$ oder $n \ddot{H}+n \vec{X}$ auftreten, wenn $H X$ eine dem Ostwald'schen Verdünnungsgesetze folgende Säure bedeutet). Dagegen erhält man selbstverständlich verschiedene Geschwindigkeitsgleichungen 
wenn in einer Reactionsgleichung undissociierte Molekeln, in einer zweiten bloß ein Theil der daraus entstehenden Ionen auftreten.

(Bei starken Elektrolyten wäre eventuell die van t'Hoff'sche Verdünnungsformel zu benutzen, wenn man annehmen will, dass sie eine Ausnahme vom Massenwirkungsgesetze bilden, beziehungsweise, dass ihr Dissociationsgrad ebenfalls durch das Verhältnis zwischen der molecularen Leitfähigkeit bei der betrachteten und bei unendlicher Verdünnung gegeben ist. Für Salzsäure gibt die van t'Hoff'sche Formel unter der Voraussetzung, dass die beiden Ionenarten mit gleichen Exponenten in der Gleichgewichtsbedingung auftreten, $\mathrm{C}_{\mathrm{H}}^{3} \mathrm{C}_{\mathrm{Cl}}^{3}=x_{\mathrm{HCl}}^{4}$. Man bekäme also identische Geschwindigkeitsgleichungen, wenn $4 \mathrm{HCl}$ durch $3 \stackrel{+}{\mathrm{H}}+3 \overline{\mathrm{C}}$ ersetzt wird.)

\$. 66. Falls zu den stattfindenden Reactionen auch unendlich rasch sich einstellende Gleichgewichte gehören, lässt sich in der Regel das System simultaner Differentialgleichungen, welches den gesammten Reactionsverlauf darstellt, vereinfachen, indem man die Gleichgewichtsbedingungen, welche aus den unendlich rasch verlaufenden Reactionen folgen, und (statt der Concentrationen der einzelnen Molekelarten) die Gesammtconcentrationen der hylotropen Gruppen in die Gleichungen der langsamer verlaufenden Reactionen einführt. Hiedurch erzielt man die Beseitigung der unendlich groß werdenden Geschwindigkeitscoefficienten und die Verminderung der Zahl der Differentialgleichungen.

Ich führe diese Umformung nicht in allgemeiner Form durch, da sie wenig übersichtlich ausfallen würde und verweise auf die im folgenden gegebenen Beispiele $(\$ .38 e$ und §. 79).

\section{Besondere Fälle.}

§. 7. Man überzeugt sich leicht, dass das im vorigen Abschnitte gegebene Schema alle Fälle der chemischen Kinetik homogener Systeme umfasst.

Eine einzige Reaction ohne katalytische Beeinflussung erhält man, wenn alle Reactionsgleichungen bis auf eine durch Nullwerden der zugehörigen Geschwindigkeitscoefficienten 
wegfallen und wenn in der übrig bleibenden Gleichung für jeden Stoff die Coefficienten nur auf einer Seite der Gleichung von Null verschieden sind. Sind die Geschwindigkeitscoefficienten $k_{2}, k_{3}$ u. S. w. Null, so ist $y=z=\ldots=0$.

Sollen z. B. Zwei Molekelarten $M_{1}$ und $M_{2}$ sich in zwei andere, $M_{3}$ und $M_{4}$ umwandeln, so sind nur $n_{1}, n_{2}, \alpha_{3}, \alpha_{4}$ von Null verschieden, dagegen $n_{3}, n_{4}$ u. s. w., ferner $\alpha_{1}, \alpha_{2}, \alpha_{5}$ u. S. w. gleich Null. Dann wird $\xi_{1}=n_{1} x, \xi_{2}=n_{2} x, \xi_{3}=-\alpha_{3} x, \xi_{4}=-\alpha_{4} x$; alle andern $\xi \operatorname{sind}$ Null. Ferner ist nach Gleichung 2 und $3(\S .4$ und 6 )

$$
\frac{d x}{d t}=k_{1}\left(A_{1}-n_{1} x\right)^{n_{1}}\left(A_{2}-n_{2} x\right)^{n_{2}}
$$

Die Stoffe, welche bei der Reaction nicht verbraucht werden, geben in der Geschwindigkeitsgleichung einen Factor mit dem Exponenten Null, dessen Wert also gleich eins ist.

§. 8. Nebenwirkungen liegen vor, wenn die Coefficienten für dasselbe $M$ auf den linken Seiten von zwei oder mehreren Reactionsgleichungen von Null verschieden sind und wenn außerdem auf sämmtlichen rechten Seiten der Reactionsgleichungen die Coefficienten aller $M$ Null werden, deren Coefficienten auf den linken Seiten von Null verschieden sind.

Folgewirkungen liegen vor, wenn ein und dasselbe $M$ sowohl auf einer rechten, als auch auf einer linken Seite zweier Reactionsgleichungen von Null verschiedene Coefficienten hat. Als besonderer Fall der Folgewirkungen erscheinen die Gegenwirkungen.

Handelt es sich im ganzen um drei Molekelarten $M_{1}, M_{2}, M_{3}$, so erhält man beispielsweise Nebenwirkungen erster Ordnung, wenn die Coefficienten folgende Werte haben:

$n_{1}=o_{1}=\alpha_{2}=\beta_{3}=1, n_{2}=n_{3}=\alpha_{1}=\alpha_{3}=o_{2}=o_{3}=\beta_{1}=\beta_{2}=0$, und wenn ferner alle $p, \gamma$ u. s. w. Null sind. Die Reactionsgleichungen sind dann

Ferner ist

$$
M_{1} \rightarrow M_{3} \quad \text { und } \quad M_{1} \rightarrow \dot{M}_{3} .
$$

$$
\begin{gathered}
\xi_{1}=x+y, \xi_{2}=-x, \xi_{3}=-y, \\
\frac{d x}{d t}=k_{1}\left(A_{1}-x-y\right), \quad \frac{d y}{d t}=k_{2}\left(A_{1}-x-y\right),
\end{gathered}
$$


daher

$$
\frac{d(x+y)}{d t}=\frac{d \xi_{1}}{d t}=\left(k_{1}+k_{2}\right)\left(A_{1}-\xi_{1}\right)
$$

Der bei der Verseifung der. Ester symmetrischer zweibasischer Säuren vorliegende Fall von Folgewirkung ergibt sich, wenn $M_{1}$ den Neutralester, $M_{2}$ das Ion der Estersäure, $M_{3}$ das Ion der freien Säure, $M_{4}$ Hydroxylionen und $M_{5}$ den Alkohol bedeuten und wenn man setzt

$$
\begin{aligned}
& n_{1}=n_{4}=1, n_{2}=n_{3}=n_{5}=0, \alpha_{2}=\alpha_{5}=1, \alpha_{1}=\alpha_{3}=\alpha_{4}=0, \\
& o_{2}=o_{4}=1, o_{1}=o_{3}=o_{5}=0, \beta=\beta_{5}=1, \beta_{1}=\beta_{2}=\beta_{4}=0 .
\end{aligned}
$$

Dann ist nach den Gleichungen 2 und 3

$$
\begin{aligned}
& \xi_{1}=x_{1}, \quad \xi_{2}=-x+y, \quad \xi_{3}=-y, \quad \xi_{4}=x+y, \quad \xi_{0}=-x-y, \\
& \frac{d x}{d t}=k_{1}\left(A_{1}-x\right)\left(A_{4}-x-y\right), \quad \frac{d y}{d t}=k_{3}\left(A_{2}+x-y\right)\left(A_{4}-x-y\right) .
\end{aligned}
$$

\section{Katalytische Wirkungen.}

8. 9. Das gegebene Schema schließt auch die katalytischen Wirkungen ein.

Hat eine Molekelart auf beiden Seiten einer Reactionsgleichung von Null verschiedene Coefficienten, so beeinflusst sie die Form der Geschwindigkeitsgleichung in anderer Weise, als dem Verhältnisse entspricht, in welchem sie aufgebraucht wird. Hat sie insbesondere auf beiden Seiten derselben Reactionsgleichung denselben von Null verschiedenen Coefficienten, so wird sie bei der Reaction überhaupt nicht aufgebraucht und stellt daher einen lediglich katalytisch wirkenden Stoff dar.

Sind die Coefficienten der Molekelart auf beiden Seiten der Reactionsgleichung nicht gleich, aber beide von Null verschieden, so entsteht oder verschwindet die Molekelart bei der Reaction, je nachdem der Coefficient auf der rechten Seite der Gleichung größer oder kleiner ist als auf der linken, und außerdem beeinflusst sie die Reaction katalytisch.

§. 10. Die im Sinne der hier gegebenen Formulierung geschriebenen Reactionsgleichungen, welche alle die Reaction beeinflussenden, also auch die katalytisch wirksamen Molekel- 
arten in jenem Verhältnisse enthalten, in welchem sie die Geschwindigkeitsgleichungen beeinflussen, will ich vollständige Reactionsgleichungen nennen, im Gegensatze zu den gew öh nlichen Reactionsgleichungen, welche nur die stöchiometrischen Beziehungen zwischen den verschwindenden und entstehenden Molekelarten angeben.

Für die Zuckerinversion ist beispielsweise die gewöhnliche Reactionsgleichung

$$
\mathrm{C}_{12} \mathrm{H}_{22} \mathrm{O}_{11}+\mathrm{H}_{2} \mathrm{O} \rightarrow 2 \mathrm{C}_{6} \mathrm{H}_{12} \mathrm{O}_{6} .
$$

Die vollständige Reactionsgleichung, welche auch den katalytischen Einfluss der Wasserstoffionen wiedergibt, ist dagegen

$$
\mathrm{C}_{12} \mathrm{H}_{22} \mathrm{O}_{11}+\mathrm{H}_{2} \mathrm{O}+\stackrel{+}{+} \rightarrow 2 \mathrm{C}_{6} \mathrm{H}_{12} \mathrm{O}_{6}+\stackrel{+}{\mathrm{H}} .
$$

Man hat $n_{1}=n_{2}=n_{3}=\alpha_{3}=1, \alpha_{1}=\alpha_{2}=0$.

Daraus folgt $\xi_{1}=\xi_{2}=x, \xi_{3}=0$, ferner

$$
\frac{d x}{d t}=k\left(A_{1}-x\right)\left(A_{2}-x\right) A_{3},
$$

wo $A_{3}$ die Concentration der Wasserstoffionen bedeutet.

Analog wäre die katalytische Beschleunigung, welche durch Wasserstoffionen bei der verseifenden Einwirkung des Wassers auf äthylschwefelsaures Kali hervorgerufen wird, durch die Gleichung zu formulieren:

$$
\mathrm{C}_{2} \mathrm{H}_{5} \mathrm{SO}_{4}+\mathrm{H}_{2} \mathrm{O}+\stackrel{+}{\mathrm{H}} \rightarrow \overline{\mathrm{SO}}_{4}+\mathrm{C}_{2} \mathrm{H}_{5} \mathrm{OH}+2 \stackrel{+}{\mathrm{H}},
$$

wobei der Einfachheit halber die Schwefelsäure als vollständig dissociiert angenommen ist.

\$. 11. Diese Formulierung der katalytischen Wirkung bietet auch Raum für die Annahme, dass die Wirkung des Katalysators nicht nach der ersten Potenz seiner Concentration, sondern nach einer höheren Potenz ansteigt. Man hat nur anzunehmen, dass der Katalysator auf beiden Seiten der Reactionsgleichung mit einem Coefficienten vorkommt, der größer ist als eins. 
§. 12. Verläuft eine Reaction auch ohne Gegenwart des Katalysators und wird durch letzteren nur beschleunigt, so kann das Stattinden von $z$ wei Reactionen angenommen werden, die im Verhältnisse von Nebenwirkungen stehen, z. B.

$$
M_{1} \rightarrow M_{2} \text { und } M_{1}+M_{3} \rightarrow M_{2}+M_{3} \text {. }
$$

Diese vollständigen Reactionsgleichungen führen zu

$$
\frac{d x}{d t}=k_{1}\left(A_{1}-\xi_{1}\right) \quad \text { und } \quad \frac{d y}{d t}=k_{2}\left(A_{1}-\xi_{1}\right) A_{3} \text {. }
$$

Daher ist $\frac{d(x+y)}{d t}=\frac{d \xi_{1}}{d t}=\left(k_{1}+k_{2} A_{3}\right)\left(A_{1}-\xi_{1}\right)$. Man bekommt also die gewöhnlich angenommene Form der Gleichung für katalytische Beschleunigungen. ${ }^{1}$

§. 13. Katalytische Verzögerungen können formal durch negative $k_{2}$ ausgedrückt werden. Ein negatives $k_{2}$ bedeutet aber, dass die Mengen der gebildeten Producte ihren eigenen Concentrationen proportional sind, während nach dem Massenwirkungsgesetze der Umsatz proportional den Mengen der verschwindenden Stoffe erfolgt. Es scheint, dass diese Schwierigkeit nur durch Vorstellungen gehoben werden kann, die darauf hinauslaufen, dass man den Einfluss gelöster verzögernder Katalysatoren als Veränderung des Mediums oder als Einwirkung auf nicht isolierbare $Z$ wischenstufen ${ }^{2}$ betrachtet. Ich gehe darauf nicht ein, da ein experimenteller Nachweis dafür, dass gelöste verzögernde Katalysatoren den Geschwindigkeitscoefficienten auf die Form $k_{1}-k_{2} A_{3}$ bringen, noch nicht erbracht zu sein scheint.

§. 14. Die hier gegebene rein formale Auffassung der katalytischen Wirkung deckt sich im wesentlichen mit jener atomistisch-kinetischen Auffassung, welche ich in einer vor kurzem erschienenen Abhandlung ${ }^{3}$ entwickelt habe. Diese kinetische Auffassung beruht auf der Annahme, dass der Katalysator mit den reagierenden Molekeln oder mit den Zwischenstufen,

1 Ostwald, Lehrb. der allg. Chemie, 2. Auflage, II, 2, S. 262 (1897).

2 Monatshefte für Chemie, 21, 385 (1900).

3 Über die Umlagerung des Cinchọins (ein Beitrag zur Theorie der katalytischen Wirkung). Monatshefte für Chemie, 21, 385 (1900), Z. f. physikal. Chemie, 34, 290. 
welche die Molekeln bei der Reaction zu durchlaufen haben, selbst in Reaction tritt.

Auch nach der kinetischen Auffassung ist es möglich, dass ein Katalysator in die Gleichung für die Reactionsgeschwindigkeit nicht mit der ersten, sondern mit einer höheren Potenz der Concentration eintritt. ${ }^{1}$

Ebenso führt die kinetische Auffassung zu der im $\S .12$ gemachten Annahme, dass, wenn eine Reaction auch ohne $\mathrm{Zu}$ satz eines Katalysators eintritt und durch letzteren beschleunigt wird, der beschleunigende Vorgang als Nebenwirkung aufzufassen ist. Es treten zwei Reactionen nebeneinander ein, von denen die eine vom Katalysator nicht beeinflusst wird, während die zweite ohne seine Gegenwart nicht möglich ist; beide führen dieselben Ausgangsstoffe in dieselben Producte über.

Endlich muss auch die kinetische Betrachtungsweise katalytische Verzögerungen als "Einfluss des Mediums « oder als Einwirkungen des Katalysators auf intermediäre Umwandlungsproducte auffassen. Die letztere Auffassung stellt eigentlich auch einen Einfluss des Mediums dar, indem sie darauf hinausläuft, dass durch den verzögernden Katalysator der reactionsfähige (active) Antheil des katalytisch beeinflussten Körpers vermindert wird.

Diese Übereinstimmung der atomistisch-kinetischen und der hier gegebenen formalen Darstellung beruht darauf, dass die Einbeziehung der Katalysatoren in die Reactionsgleichungen ebenso wie die kinetische Auffassung den Gedanken zum Ausdrucke bringt, die Katalysatoren seien nach dem Massenwirkungsgesetze an der Reaction betheiligt.

\section{Constante Verhältnisse zwischen den Geschwindigkeiten zweier nebeneinander verlaufender Reactionen.}

§. 15. Um zu zeigen, wie mit Hilfe der gegebenen allgemeinen Formulierung der Gesetze der chemischen Kinetik homogener Systeme bei constantem Volum ermittelt werden

1 Eine andere Deutung der kataiytischen Wirkungen nach höheren Potenzen der Concentration des Katalysators findet sich bei Noyes, Z. f. physikal. Chemie, 19, 599 (1896). 
kann, welche Formen von Reactionsgleichungen bestimmten Bedingungen genügen, soll nunmehr die Frage untersucht werden: In welchen Fällen ist das Verhältnis der Geschwindigkeiten zweier nebeneinander verlaufender Reactionen von der Zeit unabhängig?

Es wird nicht vorausgesetzt, dass nur diese zwei Reactionen stattfinden.

§. 16. Sollen etwa die Geschwindigkeiten der Reactionen I und II (§.1) in einem constanten (von der Zeit unabhängigen) Verhältnisse stehen, so muss die Gleichung $\frac{d x}{d t}: \frac{d y}{d t}=$ Const. erfü1lt sein.

Die Einsetzung der Werte aus den Gleichungen 3) (\$.6) ergibt

$$
\frac{k_{1}}{k_{2}}\left(A_{1}-\xi_{1}\right)^{n_{1}-o_{1}}\left(A_{2}-\xi_{2}\right)^{n_{2}-o_{2}}\left(A_{3}-\xi_{3}\right)^{n_{3}-o_{3}} \ldots=K
$$

wo $K$ von der Zeit unabhängig ist.

Diese Gleichung muss für alle Zeiten, daher auch für $t=0$ gelten. Daraus folgt

$$
K=\frac{k_{1}}{k_{2}} A_{1}^{n_{1}-o_{1}} A_{2}^{n_{2}-o_{:}} A_{3}^{n_{3}-o_{3}} \ldots
$$

§. 17. Die Erfüllung der Gleichung 4) wird nicht behindert durch jene Molekelarten, deren Concentration unveränderlich ist.

Unveränderliche Concentration einer Molekelart kann in zwei Fällen vorliegen: 1. wenn diese Molekelart bei keiner der im System stattfindenden Reactionen gebildet oder verbraucht wird, wenn also diese Molekelart bei jenen Reactionen, an denen sie überhaupt betheiligt ist, lediglich als Katalysator wirkt; 2. wenn diese Molekelart bei einem Theile der Reactionen gebildet, bei einem anderen Theile verbraucht wird, und zwar derart, dass in jedem Augenblicke die gesammte Concentrationsänderung dieser Molekelart Null ist. Unter welchen Umständen dieser Fall eintreten kann, wird im Abschnitte $V$ untersucht werden. 
Annähernd unveränderliche Concentration einer Molekelart liegt ferner vor, wenn sie in großem Überschusse vorhanden ist. Dann kann das betreffende $\xi$ neben $\operatorname{dem} A$ vernachlässigt werden.

\$. 18. Die Gleichung 4) wird befriedigt, wenn $\frac{k_{1}}{k_{2}}$ Null oder unendlich ist, wenn also eine der beiden Geschwindigkeitsconstanten Null oder unendlich ist.

§. 19. Ist das Verhältnis der Geschwindigkeitsconstanten ein endliches, so kann die Gleichung 4) nur befriedigt werden, wenn die Concentrationen (die $A-\xi$ ) aller Molekelarten, deren Concentration nicht (entsprechend den im \$. 17 erwähnten Fällen) unveränderlich ist, herausfallen. Das kann wieder auf zwei Arten geschehen: 1. indem die Exponenten jener Factoren, deren $A-\xi$ als mit der Zeit veränderlich zu betrachten ist, gleich Null werden; 2. indem für jenen Theil der Factoren, deren Exponenten nicht Null sind und deren $A-\xi$ von der Zeit abhängen, die Bedingung $\left(A_{1}-\xi_{1}\right)^{n_{1}-o_{1}}\left(A_{2}-\xi_{2}\right)^{n_{2}-o_{3}} \ldots=$ $=$ Const. in anderer Weise erfüllt wird.

§. 20. Der erste der im vorigen Paragraphen erwähnten Fälle gibt die Bedingungen

$$
n_{1}=o_{1}, n_{2}=o_{2} \text { u. s. w. }
$$

Das heißt: Die linken Seiten der vollständigen Reactionsgleichungen müssen hinsichtlich der Molekelarten von veränderlicher Concentration identisch sein.

Wenn für alle Molekelarten diese Bedingung erfüllt ist, so wird $K=\frac{k_{1}}{k_{2}}$; das Verhältnis der Reactionsgeschwindigkeiten ist in jedem Augenblicke gleich dem der Geschwindigkeitsconstanten, unabhängig von den Anfangsmengen der reagierenden Stoffe.

§. 21. Die Gleichungen der beiden Reactionen mit constantem Geschwindigkeitsverhältnisse haben dann die Form

$$
\begin{aligned}
& n_{1} M_{1}+n_{2} M_{2}+\ldots \rightarrow \alpha_{1} M_{1}+\alpha_{2} M_{2}+\ldots \\
& n_{1} M_{1}+n_{2} M_{2}+\ldots \rightarrow \beta_{1} M_{1}+\beta_{2} M_{2}+\ldots
\end{aligned}
$$


Hiebei sind folgende Fälle zu unterscheiden:

1. $n_{1}=0$. Dann wird der Stoff $M_{1}$ bei keiner der beiden Reactionen verbraucht und wirkt auch nicht katalytisch; wohl aber kann er bei einer oder bei beiden gebildet werden.

2. $n_{1}<\alpha_{1}$, aber von Null verschieden. Dann wird $M_{1}$ bei der betreffenden Reaction. gebildet und wirkt außerdem als Katalysator.

3. $n_{1}=\alpha_{1} . M_{1}$ wirkt bei dieser Reaction nur als Katalysator. Seine Menge kann aber durch andere Reactionen verändert werden.

4. $n_{1}>a_{1} . M_{1}$ wird bei der betreffenden Reaction verbraucht. Wenn $\alpha_{1}$ von Null verschieden ist, wirkt es außerdem als Katalysator.

8. 22. Fall 1 besagt, dass mit dem Auftreten eines constanten Geschwindigkeitsverhältnisses zwischen zwei Reactionen beliebige Stoffe verträglich sind, welche nur auf den rechten Seiten der zwei vollständigen Reactionsgleichungen vorkommen, also bei diesen Reactionen weder katalytisch wirken, noch verbraucht werden. Das ist selbstverständlich, weil solche Stoffe auf die beiden Geschwindigkeitsgleichungen keinen Einfluss haben.

§. 23. Die Combinationen der Fälle 2. bis 4. geben verschiedene Reactionstypen, bei denen constante Geschwindigkeitsverhältnisse auftreten können.

Entspricht eine Molekelart in beiden Reactionsgleichungen dem Falle 4, so sind die Reactionen in Bezug auf diesen Stoff Nebenwirkungen.

Beispiele:

$$
\begin{aligned}
& \text { Vollständige } \\
& \text { Reactionsgleichung }
\end{aligned}
$$

I. $M_{1}+M_{2} \rightarrow M_{3}$

III. $M_{1}+M_{2} \rightarrow M_{4}$

II. $M_{1}+2 M_{2} \rightarrow M_{3}$

II. $M_{1}+2 M_{2} \rightarrow M_{2}+M_{4}$
Gewöhnliche Reactionsgleichung

Ebenso

$$
M_{1}+2 M_{2} \rightarrow M_{3}
$$$$
M_{1}+M_{2} \rightarrow M_{4}
$$

Katalysatoren

8. 24. In den Fällen 2 und 3 beeinflusst die betreffende Molekelart die Reactionsgeschwindigkeit nur als Katalysator. Diese Fälle können also nur zusammen mit 4 vorkommen. 
\$. 25. Combinationen der Fälle 3 und 4 ergeben $\mathrm{Neben-}$ wirkungen hinsichtlich eines Theiles der reagierenden Stoffe, verbunden mit katalytischen Beeinflussungen, oder Reactionspaare, deren gewöhnliche Reactionsgleichungen entweder durchaus verschiedene Stoffe enthalten oder nur entstehende Stoffe gemeinsam haben, welche aber infolge katalytischer Wirkungen identische linke Seiten der vollständigen Reactionsgleichungen haben.

Beispiele:

Vollständige

Reactionsgleichung

I I. $M_{1}+M_{2} \rightarrow M_{3}$

III. $M_{1}+M_{2} \rightarrow M_{2}+M_{4}$

\{. I. $M_{1}+M_{2} \rightarrow M_{1}+M_{3}$

III. $\quad M_{1}+M_{2} \rightarrow M_{2}+M_{4}$

\{ I. $\quad M_{1}+M_{2} \rightarrow M_{2}+M_{3}$

II. $\quad M_{1}+M_{2} \rightarrow M_{1}+M_{3}$
Gewöhnliche

Reactionsgleichung

$M_{1}+M_{2} \rightarrow M_{3}$

$M_{1} \rightarrow M_{4}$

$M_{2} \rightarrow M_{3}$

$M_{1} \rightarrow M_{4}$

$M_{1} \rightarrow M_{3}$

$M_{2} \rightarrow M_{3}$
Katalysatoren

$M_{2}$

$M_{1}$

$M_{2}$

$M_{2}$

$M_{1}$

\$. 26. Combinationen der Fälle 2 und 4 (eventuell neben 3) geben Folgewirkungen. Denn dann wird der Stoff bei der einen Reaction gebildet und bei der anderen verbraucht und

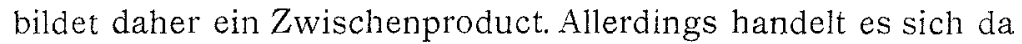
um Folgewirkungen von ganz besonderer Art, für die es bisher kein Beispiel zu geben scheint. Bei der Reaction, welche dem Falle 2 entspricht, muss das Zwischenproduct zugleich als Katalysator wirken, derart, dass die Reaction bei Abwesenheit des Katalysators überhaupt nicht eintritt. Ist der betreffende Stoff nicht vom Anfang an da, so treten beide Reactionen nicht ein. Ist aber der Stoff vom Anfang an da, so kann die Reaction, bei welcher er verbraucht wird, schon beginnen, ehe durch die andere Reaction merkliche Mengen dieses Stoffes gebildet werden.

Beispiel.

Vollständige Reactionsgleichung

I. $\quad M_{1}+M_{2} \rightarrow 2 M_{2}$

II. $\quad M_{1}+M_{2} \rightarrow M_{1}+M_{3}$

Chemie-Heft Nr. 8.
Gewöhnliche

Reactionsgleichung

$$
M_{1} \rightarrow M_{2}
$$$$
M_{2} \rightarrow M_{3}
$$

Katalysator

$M_{2}$

$M_{1}$ 
Es ist ersichtlich, dass es sich um eine Folgewirkung handelt, bei der $M_{2}$ das Zwischenproduct ist. Aber bei Abwesenheit von $M_{2}$ tritt gar keine Reaction ein und bei Zusatz von $M_{2}$ ist die $z$ weite Reaction auf die Bildung von $M_{2}$ durch die erste Reaction nicht angewiesen; wohl aber wird ihr Verlauf dadurch beeinflusst.

Als specieller Fall derFolgewirkungen können auch Gegenwirkungen hieher gehören.

Beispiel. ${ }^{1}$

$$
\begin{gathered}
\text { Vollständige } \\
\text { Reactionsgleichung } \\
M_{1}+M_{2} \rightarrow 2 M_{1} \\
M_{1}+M_{2} \rightarrow 2 M_{2}
\end{gathered}
$$

$$
\begin{gathered}
\begin{array}{c}
\text { Gewöhnliche } \\
\text { Reactionsglëichung }
\end{array} \\
M_{2} \rightarrow M_{1} \\
M_{1} \rightarrow M_{2}
\end{gathered}
$$

Katalysator

$M_{1}$

$M_{2}$

§. $26 a$. Gemäß §. $6 a$ können in einem Theile der Reactionsgleichungen Molekelarten oder Gruppen von Molekelarten durch andere ersetzt werden, welche mit ihnen durch ein sich unendlich rasch einstellendes Gleichgewicht verknüpft sind, ohne dass dadurch das constante Geschwindigkeitsverhältnis gestört wird.

Das sich unendlich rasch einstellende Gleichgewicht entspreche der Gleichung

$$
\left(A_{1}-\xi_{1}\right)^{n_{1}}\left(A_{2}-\xi_{2}\right)^{n_{\mathrm{\nu}}} \ldots=\chi_{(}\left(A_{\nu+1}-\xi_{\mathrm{y}+1}\right)^{n_{\nu}+1}\left(A_{\nu+2}-\xi_{\mathrm{\nu}+2}\right)^{n_{\nu}+2 \ldots}
$$

Dann herrscht ein constantes Geschwindigkeitsverhältnis nicht bloß zwischen den Reactionen

$$
\text { I. } \quad n_{1} M_{1}+\dot{n}_{2} M_{2}+\ldots+X \rightarrow Y
$$

und

$$
\text { II. } n_{1} M_{1}+n_{2} M_{2}+\ldots+X \rightarrow Z \text {, }
$$

. sondern auch zwischen den Reactionen

$$
\mathrm{I}^{\prime} . \quad n_{\nu+1} M_{\nu+1}+n_{\nu+2} M_{\nu+2}+\ldots+X \rightarrow Y
$$

und

$$
\text { II. } n_{1} M_{1}+n_{2} M_{2}+\ldots+X \rightarrow Z \text {. }
$$

1 Dieses Reactionspaar hat merkwürdige Eigenschaften. Für $k_{1}=k_{2}$ befinden sich die Stoffe $M_{1}$ und $\mathrm{M}_{2}$ in jedem Verhältnisse im Gleichgewichte. Für $k_{1} \gtrless k_{2}$ tritt vollständiger Umsatz in einem oder anderen Sinne eir. 
$X$ bedeutet Glieder, die sich auf nicht dem Gleichgewichte angehörige Molekelarten beziehen. In letzterem Falle geht Gleichung 4) über in

$$
K=\frac{k_{1}^{\prime}\left(A_{v+1}-\xi_{v}+1\right)^{n_{\nu}+1} \ldots}{k_{2}\left(A_{1}-\xi_{1}\right)^{n_{1}} \ldots},
$$

wo $k_{1}^{\prime}$ die Geschwindigkeitsconstante der Reaction I' ist. Daraus folgt $K=\frac{k_{1}^{\prime}}{k_{2} x}$, während beim Reactionspaare I und II $K=\frac{k_{1}}{k_{2}}$ ist. Sind die linken Seiten dervollständigen Reactionsgleichungen durch die in ihnen auftretenden hylotropen Formen verschieden, sonst aber identisch, so tritt in den Ausdruck für das Geschwindigkeitsverhältnis auch die Gleichgewichtsconstante der hylotropen Gruppe ein.

Der Zahlenwert des Geschwindigkeitsverhältnisses ist selbstverständig davon unabhängig, ob man bei der Berechnung der ersten Reaction die Reactionsgleichung I oder $\mathrm{I}^{\prime}$ zugrunde legt. Die errechnete Geschwindigkeitsconstante ist entweder $k_{1}$ oder $k_{1}^{\prime}=k_{1} x$ (vergl. \$. $6 a$ ). $K$ bleibt immer gleich $\frac{k_{1}}{k_{2}}$.

Dass dem $\$ .6$ a entsprechende hylotrope Formen wie identische Molekelarten zu behandeln sind, ist auch im folgenden festzuhalten.

Ein Beispiel für diesen Fall folgt im $\S .38$ b.

§. 27. Im vorstehenden ist die Bedingung entwickelt worden, welcher die beiden Reactionsgleichungen genügen müssen, damit ein constantes Geschwindigkeitsverhältnis entsprechend dem ersten, im $\S .19$ erwähnten Falle auftritt. Die Bedingung besteht in der Gleichheit der linken Seiten der vollständigen Reactionsgleichungen hinsichtlich der Molekelarten von veränderlicher Concentration.

Es ist nunmehr noch $z u$ untersuchen, in welchen. Fällen constante Geschwindigkeitsverhältnisse, entsprechend dem zweiten im §. 19 erwähnten Falle auftreten. Es muss also

$$
\left(A_{1}-\xi_{1}\right)^{n_{1}-o_{1}}\left(A_{2}-\xi_{2}\right)^{n_{2}-o_{2}} \ldots=\text { Const. }
$$

sein, ohne dass $n_{1}=o_{1}, n_{2}=o_{2} \ldots$ ist.

In der Regel ist Gleichung 6) bei veränderlichem $\xi$ nur erfüllbar, wenn Gleichungen von folgender Form gelten:

$$
A_{1}-\xi_{1}=C_{1} \%(t), A_{2}-\xi_{2}=C_{2}[\varphi(t)]^{\text {vg }_{2}} \text { u. s. w.; }
$$


wo $(t)$ eine und dieselbe Function der Zeit ist, während die $C$ und $\nu$ constant sind. Dann geht Gleichung 6 ) über in

$$
C_{1}^{n_{1}-o_{1}} C_{2}^{n_{2}-o_{2}} \ldots \varphi(t)^{\left(n_{1} \cdots o_{1}\right)+v_{3}\left(t_{2}-\cdots o_{3}\right)+\cdots}=\text { Const. }
$$

Diese Gleichung wird erfüllt durch

$$
\left(n_{1}-o_{1}\right)+v_{2}\left(n_{2}-o_{2}\right)+v_{3}\left(n_{3}-o_{3}\right)+\ldots=0 .
$$

Selbstverständlich können die Factoren der Gleichung (b) auch gruppenweise den Gleichungen 7) und 8) genügen, wodurch dann ebenfalls die Gleichung 6 ) befriedigt wird.

Aufösungen der Gleichung 8) sind immer möglich. Unter diese Lösungen gehören auch $n_{1}-o_{1}=0 \mathrm{u}$. s. w., welcher Fall bereits im vorigen behandelt ist.

Lösungen wie $\nu_{2}=0$ bieten ebenfalls nichts Neues, da sie zu $A_{2}-\xi_{2}=C_{2}$ führen, also angeben, dass die betreffende Molekelart von unveränderlicher Concentration ist, was bereits in $\$ .17$ besprochen wurde.

In dẹr Regel sind jedoch auch andere Lösungen möglich.

8. 28. Die Constanten $C$ in den Gleichungen 7) können in folgender Weise ausgewertet werden.

Diese Gleichungen müssen für jedes $t$ gelten. Für $t=0$ ist $\xi_{1}=\xi_{2}=\ldots=0$, daher

$$
C_{1}=\frac{A_{1}}{\varphi(0)}, \quad C_{2}=\frac{A_{2}}{[\varphi(0)]^{\nu_{3}}}, \quad C_{3}=\frac{A_{3}}{[\varphi(0)]^{\nu_{3}}} \ldots
$$

Somit ist

$$
A_{1}-\xi_{1}=A_{1} \frac{\varphi(t)}{\varphi(0)}, \quad A_{2}-\xi_{2}=A_{2}\left[\frac{\varphi(t)}{\varphi(0)}\right]^{v_{2}} \text { u. s. w. }
$$

Ferner

$$
\left.\xi_{1}=A_{1}\left\{1-\frac{\varphi(t)}{\varphi(0)}\right\}, \quad \xi_{2}=A_{2}\left\{1-\mid \frac{\varphi(t)}{\varphi(0)}\right]^{\gamma_{2}}\right\} \text { u. s. w. 10) }
$$

ร. 29. Die Erfüllung der Gleichungen 8) und 9) ist hinreichend, um das Geschwindigkeitsverhältnis der beiden Reactionen constant $z u$ machen.

Die Gleichungen 9) werden jedoch nur in verhältnismäßig seitenen Fällen erfüllt sein. Ich bin nicht in der. Lage, die Formen von Reactionsgleichungen, bei denen die Gleichungen 9 ) zutreffen, systematisch zu entwickeln. Jedenfalls kommt es aber hier nicht nur (wie bei dem im $\$ 20$ behandelten Falle) 
auf die zwei Reactionsgleichungen an, deren Geschwindigkeitsverhältnis constant sein soll, sondern auf alle daneben verlaufenden Reactionen, welche die Concentrationen (die $\xi$ ) der in den beiden betrachteten Geschwindigkeitsgleichungen vorkommenden Stoffe verändern.

§. 30. Es lässt sich jedoch leicht zeigen, dass eine nothwendige (aber noch nichthinreichende) Bedingung für die Existenz einer die Gleichungen 9 ) befriedigenden Function $\varphi(t)$ in dem Stattfinden gewisser Bezieh ungen $z$ wischen den in den Geschwindigkeitsgleichungen aller nach $\S .29$ zu berücksichtigenden Reactionen vorkommenden Constanten (Geschwindigkeitscoefficienten, Anfangsconcentrationen, Coefficienten der Reactionsgleichungen) besteht.

Angenommen, es existiere eine Function $\varphi(t)$, welche die Gleichungen 9) erfüllt, so ergibt sich durch Differentiation der Gleichungen 10)

$$
\begin{gathered}
\frac{d \xi_{1}}{d t}=-A_{1} \frac{\varphi^{\prime}(t)}{\varphi(0)}, \quad \frac{d \hat{\xi}_{2}}{d t}=-\nu_{2} A_{2}\left[\frac{\varphi(t)}{\varphi(0)}\right]^{\nu_{2}-1} \frac{\varphi^{\prime}(t)}{\varphi(0)}, \\
\frac{d \dot{\xi}_{3}}{d t}=-\nu_{3} A_{3}\left[\frac{\varphi(t)}{\varphi(0)}\right]^{\nu_{3}-1} \frac{\varphi^{\prime}(t)}{\varphi(0)} \text { u. s. w. }
\end{gathered}
$$

Daraus folgt

$\frac{d \xi_{2}}{d t}: \frac{d \xi_{1}}{d t}=\frac{\nu_{2} A_{2}}{A_{1}}\left[\frac{\varphi(t)}{\varphi(0)}\right]^{\nu_{2}-1}, \frac{d \xi_{3}}{d t}: \frac{d \xi_{1}}{d t}=\frac{\nu_{3} A_{3}}{A_{1}}\left[\frac{\varphi(t)}{\varphi(0)}\right]^{\gamma_{3}-1}$ u. s.w.

Diese Gleichungen müssen für jeden Wert von $t$ gelten, daher auch für $t=0$. Für diesen Fall gehen sie über in

$$
\left(\frac{d \xi_{2}}{d t}: \frac{d \xi_{1}}{d t}\right)_{t=0}=\frac{v_{2} A_{2}}{A_{1}},\left(\frac{d \xi_{3}}{d t}: \frac{d \xi_{1}}{d t}\right)_{t=0}=\frac{\nu_{3} A_{3}}{A_{1}} \text { u.s.w. 11) }
$$

Für die gleichen Quotienten lassen sich aber Ausdrücke gewinnen, welche unabhängig von jeder besonderen Voraussetzung giltig sind.

Differenziert man die Gleichungen 2) (\$.4), so erhält man

$$
\frac{d \xi_{2}}{d t}: \frac{d \xi_{1}}{d t}=\frac{\left(n_{2}-\alpha_{2}\right) \frac{d x}{d t}+\left(o_{2}-\beta_{2}\right) \frac{d y}{d t}+\left(p_{2}-\gamma_{2}\right) \frac{d z}{d t}+\ldots}{\left(n_{1}-\alpha_{1}\right) \frac{d x}{d t}+\left(o_{1}-\beta_{1}\right) \frac{d y}{d t}+\left(p_{1}-\gamma_{1}\right) \frac{d z}{d t}+\ldots}
$$

tu. S. W, 
Aus den Gleichungen 3) (\$.6) ergeben sich die Werte, welche $\frac{d x}{d t}, \frac{d y}{d t} \ldots$ für $t=0$ annehmen.

Man hat

$$
\begin{aligned}
& \left(\frac{d x}{d t}\right)_{t=0}=k_{1} A_{1}^{i_{1}} A_{2}^{n_{2}} \ldots \\
& \left(\frac{d y}{d t}\right)_{t=0}=k_{2} A_{1}^{o_{1}} A_{2}^{o_{\mathrm{a}}} \cdots
\end{aligned}
$$

Daher ist

$$
\begin{aligned}
& \left(\frac{d \xi_{2}}{d t}: \frac{d \xi_{1}}{d t}\right)_{t=0}=\frac{\left(n_{2}-\alpha_{2}\right) k_{1} A_{1}^{n_{1}} A_{2}^{n_{2}} \ldots+\left(o_{2}-\beta_{2}\right) k_{2} A_{1}^{o_{1}} A_{2}^{o_{2}} \ldots+. .}{\left(n_{1}-\alpha_{1}\right) k_{1} A_{1}^{n_{1}} A_{2}^{n_{2}} \ldots+\left(o_{1}-\beta_{1}\right) k_{2} A_{1}^{o_{1}} A_{2}^{o_{2}} \ldots+\ldots} \\
& \left(\frac{d \xi_{3}}{d t}: \frac{d \xi_{1}}{d t}\right)_{t=0}=\frac{\left(n_{3}-\alpha_{3}\right) k_{1} A_{1}^{n_{1}} A_{2}^{n_{2}} \ldots+\left(o_{3}-\beta_{3}\right) k_{2} A_{1}^{o_{1}} A_{2}^{o_{2}} \ldots+\ldots}{\left(n_{1}-\alpha_{1}\right) k_{1} A_{1}^{n_{1}} A_{2}^{n_{2}} \ldots+\left(o_{1}-\beta_{1}\right) k_{2} A_{1}^{o_{1}} A_{2}^{o_{2}} \ldots+\ldots}
\end{aligned}
$$

u. s. w.

Vergleicht man diese Ausdrücke mit den Gleichungen 11), so folgt, dass eine die Gleichungen 9) befriedigende Function $\varphi(t)$ nur existieren kann, wenn folgende Gleichungen erfüllt sind:

$$
\left.\begin{array}{l}
\nu_{2}=\frac{\left(n_{2}-\alpha_{2}\right) k_{1} A_{1}^{n_{1}} A_{2}^{n_{2}-1} A_{3}^{n_{3}} \ldots+\left(o_{2}-\beta_{2}\right) k_{2} A_{1}^{o_{1}} A_{2}^{o_{2}-1} A_{3}^{o_{3}} \ldots+\ldots}{\left(n_{1}-\alpha_{1}\right) k_{1} A_{1}^{n_{1}-1} A_{2}^{n_{2}} A_{3}^{n_{3}} \ldots+\left(o_{1}-\beta_{1}\right) k_{2} A_{1}^{o_{1}-1} A_{2}^{o_{2}} A_{3}^{o_{3}} \ldots+\ldots} \\
\nu_{3}=\frac{\left(n_{3}-\alpha_{3}\right) k_{1} A_{1}^{n_{1}} A_{2}^{n_{2}} A_{3}^{n_{3}-1} \ldots+\left(o_{3}-\beta_{3}\right) k_{2} A_{1}^{o_{1}} A_{2}^{o_{2}} A_{3}^{o_{3}-1} \ldots+\ldots}{\left(n_{1}-\alpha_{1}\right) k_{1} A_{1}^{n_{1}-1} A_{2}^{n_{2}} A_{3}^{n_{3}} \ldots+\left(o_{1}-\beta_{1}\right) k_{2} A_{1}^{o_{1}-1} A_{2}^{o_{2}} A_{3}^{o_{3}} \ldots+\ldots}
\end{array}\right\}
$$

Es müssen aber auch bei Erfüllung dièser Gleichungen nicht nothwendig die Gleichungen 9 ) befriedigt sein

Da zu den Gleichungen 12) noch die Gleichung 8) hinzutritt, hat man eine Gleichung mehr, als die Zahl der $v$ beträgt. Durch Einsetzung der y-Werte aus 12) in 8) erhält man eine Gleichung, welche erfüllt sein muss, wenn die Geschwindigkeiten der Reactionen I und II in einem constanten Verhältnisse stehen sollen. Diese Gleichung drückt eine Beziehüng zwischen den $k, A, n, o \ldots, x, \beta \ldots$ aus und lautet 


$$
\begin{gathered}
0=\left(n_{1}-o_{1}\right)\left[\left(n_{1}-\alpha_{1}\right) k_{1} A_{1}^{n_{1}-1} A_{2}^{n_{2}} A_{3}^{n_{3}} \ldots+\right. \\
\left.+\left(o_{1}-\beta_{1}\right) k_{2} A_{1}^{o_{1}-1} A_{2}^{o_{2}} A_{3}^{o_{3}} \ldots+\ldots\right] \\
+\left(n_{2}-o_{2}\right)\left[\left(n_{2}-\alpha_{2}\right) k_{1} A_{1}^{n_{1}} A_{2}^{n_{2}-1} A_{3}^{n_{3}} \ldots+\right. \\
\left.+\left(o_{2}-\beta_{2}\right) k_{2} A_{1}^{o_{1}} A_{2}^{o_{2}-1} A_{3}^{o_{3}} \ldots+\ldots\right] \\
+\left(n_{3}-o_{3}\right)\left[\left(n_{3}-\alpha_{3}\right)\right. \\
k_{1} A_{1}^{n_{1}} A_{2}^{n_{2}} A_{3}^{n_{3}-1} \ldots+ \\
\left.+\left(o_{3}-\beta_{3}\right) k_{2} A_{1}^{o_{1}} A_{2}^{o_{2}} A_{3}^{o_{2}-1} \ldots+\ldots\right]
\end{gathered}
$$

Bei Bildung des ersten Gliedes dieses Ausdruckes sind alle nebeneinander verlaufenden Reactionen $\mathrm{zu}$ berücksichtigen, welche die Menge von $M_{1}$ verändern, ebenso für das zweite Glied alle, welche die Menge von $M_{2}$ verändern u. s. w. Die Zahl der Glieder hängt dagegen nur von jenen Molekelarten ab, welche auf den linken Seiten der vollständigen Reactionsgleichungen jener Reactionen vorkommen, zwischen denen das constante Geschwindigkeitsverhältnis bestehen soll und deren Concentration nicht unveränderlich ist. Die Gleichung schließt den Fall ein, dass die Bedingung des $\S .27$ für die Stoffe gruppenweise erfüllt ist, ferner der Fall des §. 20.

Gleichung 13) muss i m m er erfüllt sein, wenn ein constantes Geschwindigkeitsverhältnis auftreten soll. Insbesondere bleibt sie auch dann eine hiefür nothwendige Bedingung, wenn ein constantes Geschwindigkeitsverhältnis möglich sein sollte, ohne dass Gleichung 7) erfüllt ist. Man kann sie nämlich ohne jede weitere Voraussetzung aus Gleichung 4) ableiten.

Nimmt man von Gleichung 4) den Logarithmus und differenziert die so erhaltene Gleichung, so bekommt man

$$
0=\frac{n_{1}-o_{1}}{A_{1}-\xi_{1}} \frac{d \xi_{1}}{d t}+\frac{n_{2}-o_{2}}{A_{2}-\xi_{2}} \frac{\xi_{2} p}{d t}+\ldots
$$

Diese Gleichung muss für jedes $t$ erfüllt sein, wenn ein constantes Umwandlungsverhältnis auftreten soll. Setzt man in dieser Gleichung die Werte für $t=0$ ein, so erhält man Gleichung 13) als nothwendige, aber nicht hinreichende Bedingung für das Auftreten eines constanten Geschwindigkeitsverhältnisses bei beliebiger Form der Geschwindigkeitsgleichungen. 
§. 31. Als Beispiel zu dem Besprochenen soll untersucht werden, wie die Reactionsgleichungen beschaffen sein müssen, damit die Reactionsgeschwindigkeiten bei beliebigen Anfangsconcentrationen in einem von der Zeit unabhängigen Verhältnisse stehen, wenn nur $z$ wei Reactionen stattfinden und die Zahl der auf den linken Seiten der vollständigen Reactionsgleichungen vorkommenden Molekelarten im ganzen zwei ist. Die Geschwindigkeitsconstanten der beiden Reactionen sollen endliche Werte haben.

Die vollständigen Reactionsgleichungen sind dann

$$
\begin{aligned}
& n_{1} M_{1}+n_{2} M_{2} \rightarrow \alpha_{1} M_{1}+\alpha_{2} M_{2}+\alpha_{3} M_{3}+\ldots \\
& o_{1} M_{1}+o_{3} M_{2} \rightarrow \beta_{1} M_{1}+\beta_{2} M_{2}+\beta_{3} M_{3}+\ldots
\end{aligned}
$$

§. 32. Nach §. 20 tritt ein constantes Geschwindigkeitsverhältnis auf, wenn $n_{1}=o_{1}$ und $n_{2}=o_{2}$ ist. Die Beschaffenheit der Reactionspaare, welche dieser Bedingung genügen, ist bereits in $\S .21$ bis 26 erörtert worden.

Es kann aber auch ein constantes Geschwindigkeitsverbältnis entsprechend dem in $\S .27$ besprochenen Falle auftreten. Eine nothwendige, aber nicht hinreichende Bedingung dafür ist die Erfüllung der Gleichung 13) (\$. 30).

Im vorliegenden Falle nimmt die Gleichung 13) die Form an

$$
\begin{aligned}
0= & \left(n_{1}-o_{1}\right)\left[\left(n_{1}-\alpha_{1}\right) k_{1} A_{1}^{n_{1}-1} A_{2}^{n_{2}}+\left(o_{1}-\beta_{1}\right) k_{2} A_{1}^{o_{1}-1} A_{2}^{o_{2}}\right] \\
& +\left(n_{2}-o_{2}\right)\left[\left(n_{2}-\alpha_{2}\right) k_{1} A_{1}^{n_{1}} A_{2}^{n_{2}-1}+\left(o_{2}-\beta_{2}\right) k_{2} A_{1}^{o_{1}} A_{2}^{o_{2}-1}\right] .
\end{aligned}
$$

Von den Anfangsconcentrationen unabhängige Lösungen dieser Gleichung sind nur möglich, wenn entweder die vier Gleichungen

$$
\begin{array}{ll}
\left(n_{1}-o_{1}\right)\left(n_{1}-\alpha_{1}\right)=0, & \left(n_{1}-o_{1}\right)\left(o_{1}-\beta_{1}\right)=0 \\
\left(n_{2}-o_{2}\right)\left(n_{2}-\alpha_{2}\right)=0, \quad & \left(n_{2}-o_{2}\right)\left(o_{2}-\beta_{2}\right)=0
\end{array}
$$

erfüllt sind oder wenn nur ein Theil dieser Gleichungen erfüllt ist und aus den übrig bleibenden Gliedern der Gleichung 14) die $A$ durch Nullwerden ihrer Exponenten herausfallen oder durch Division weggeschafft werden können.

In den Gleichungen 15) sind die dem $\$ .20$ entsprechenden Lösungen $\left(n_{1}=o_{1}, n_{2}=o_{2}\right)$ mitenthalten. Ist etwa $n_{1}$ von $o_{1}$ 
verschieden, so können die Gleichungen 15) nur durch $n_{1}=\alpha_{1}$, $o_{1}=\beta_{1}$ befriedigt werden. Dann ist der Stoff $M_{1}$ bei beiden Reactionen nur Katalysator, also von unveränderlicher Concentration; auch dieser Fall gehört unter $\S .20$, da die Molekelarten von unveränderlicher Concentration nicht berücksichtigt zu werden brauchen (\$. 17). Die Lösung $n_{1}-\alpha_{1}=o_{1}-\beta_{1}=$ $=n_{2}-\alpha_{2}=o_{2}-\beta_{2}=0$ hat keinen Sinn, da in diesem Falle überhaupt keine Reaction möglich ist.

§. 33. Dagegen erhält man neue Lösungen, wenn nur ein Theil der Gleichungen 15) erfüllt ist. Man überzeugt sich leicht, dass nur $n_{1}-\alpha_{1}=o_{2}-\beta_{2}=0$ oder $o_{1}-\beta_{1}=n_{2}-\alpha_{2}=0$ in Betracht kommt; diese beiden Fälle führen natürlich zur selben Form der Reactionsgleichungen, da sie durch Vertauschung der gleichwertigen Indices 1 und 2 ineinander übergehen.

Setzt man $n_{1}-\alpha_{1}=o_{2}-\beta_{2}=0$, so geht Gleichung 14) über in

$$
0=\left(n_{1}-o_{1}\right)\left(o_{1}-\beta_{1}\right) k_{2} A_{1}^{o_{1}-1} A_{2}^{o_{2}}+\left(n_{2}-o_{2}\right)\left(n_{2}-\alpha_{2}\right) k_{1} A_{1}^{n_{1}} A_{2}^{n_{2}-1} .
$$

Aus dieser Gleichung lassen sich $A_{1}$ und $A_{2}$ wegschaffen, wenn man setzt $o_{1}-1=n_{1}$ und $o_{2}=n_{9}-1$.

Dann bleibt $0=\left(n_{1}-o_{1}\right)\left(o_{1}-\beta_{1}\right) k_{2}+\left(n_{2}-o_{2}\right)\left(n_{2}-\alpha_{2}\right) k_{1}$, woraus durch Einsetzung der Werte $o_{1}=n_{1}+1, o_{2}=n_{2}-1$ entsteht

$$
\frac{k_{1}}{k_{2}}=\frac{n_{1}+1-\beta_{1}}{n_{2}-\alpha_{2}}
$$

Die vollständigen Reactionsgleichungen lauten dann

$$
\begin{array}{cr}
\text { I. } & n_{1} M_{1}+n_{2} M_{2} \rightarrow n_{1} M_{1}+\alpha_{2} M_{2}+\alpha_{3} M_{3}+\ldots \\
\text { II. } \quad\left(n_{1}+1\right) M_{1}+\left(n_{2}-1\right) M_{2} \rightarrow \beta_{1} M_{1}+\left(n_{2}-1\right) M_{2}+\beta_{3} M_{3} \ldots
\end{array}
$$

In diesen Reactionsgleichungen muss jedenfalls $n_{2}>a_{2}$ und $n_{1}+1>\beta_{1}$ sein, da sonst überhaupt kein Stoff verbraucht würde.

Die gewöhnlichen Reactionsgleichungen sind

$$
\begin{array}{lr}
\text { I. } \quad\left(n_{2}-\alpha_{2}\right) M_{2} \rightarrow \alpha_{3} M_{3}+\ldots \\
\text { II. } \quad\left(n_{1}+1-\beta_{1}\right) M_{1} \rightarrow \beta_{3} M_{3}+\ldots
\end{array}
$$


Es handelt sich also um Reactionen, welche hinsichtlich der verschwindenden Stoffe völlig verschieden sind.

Wenn die vollständigen Reactionsgleichungen die hier angegebene Form haben und wenn außerdem die Geschwindigkeitsconstanten in der durch Gleichung 15 a) angegebenen Beziehung stehen, so können bei beliebigen Anfangsconcentrationen die Reactionsgeschwindigkeiten in einem von der Zeit unabhängigen Verhältnisse stehen. $\mathrm{Da}$ aber die Bedingungen des $\S 30$ nur nothwendige, aber nicht hinreichende Bedingungen für das Auftreten eines constanten Geschwindigkeitsverhältnisses sind, ist noch zu prüfen, ob die Constanz dieses Verhältnisses hier wirklich zutrifft. Im vorliegenden Falle ist das Verhältnis der Reactionsgeschwindigkeiten wirklich constant.

Die Geschwindigkeitsgleichungen sind nach \$. 6

$$
\begin{aligned}
& \frac{d x}{d t}=k_{1}\left(A_{1}-\xi_{1}\right)^{n_{1}}\left(A_{2}-\xi_{2}\right)^{n_{2}} \\
& \frac{d y}{d t}=k_{2}\left(A_{1}-\xi_{1}\right)^{n_{1}+1}\left(A_{2}-\xi_{2}\right)^{n_{2}-1} .
\end{aligned}
$$

Daher ist

$$
\frac{d y}{d t}: \frac{d x}{d t}=\frac{k_{2}}{k_{1}} \cdot \frac{A_{1}-\xi_{1}}{A_{2}-\xi_{2}} .
$$

Nach $\S .4$ ist $\xi_{1}=\left(n_{1}+1-\beta_{1}\right) y, \xi_{2}=\left(n_{2}-\alpha_{3}\right) x$.

Die Integration der Gleichung 16) gibt

$$
\begin{aligned}
\frac{1}{n_{1}+1-\beta_{1}} l \frac{A_{1}-\left(n_{1}+1-\beta_{1}\right) y}{A_{1}} & = \\
= & \frac{k_{2}}{k_{1}\left(n_{2}-\alpha_{2}\right)} l \frac{A_{2}-\left(n_{2}-\alpha_{2}\right) x}{A_{2}},
\end{aligned}
$$

woraus folgt:

$$
y=\frac{A_{1}}{n_{1}+1-\beta_{1}}\left\{1-\left[\frac{A_{2}-\left(n_{2}-\alpha_{2}\right) x}{A_{2}}\right]^{\frac{k_{2}\left(n_{1}+1-\beta_{1}\right)}{k_{1}\left(n_{2}-\alpha_{2}\right)}}\right\} .
$$

Durch Ėinführung der durch die Gleichung 15 a) ausgedrückten Bedingung folgt 


$$
y=\frac{A_{1}}{n_{1}+1-\beta_{1}}\left[1-\frac{A_{2}-\left(n_{2}-\alpha_{2}\right) x}{A_{2}}\right]=\frac{\left(n_{2}-\alpha_{2}\right) A_{1} x}{\left(n_{1}+1-\beta_{1}\right) A_{2}} .
$$

Durch Einfübrung dieses Wertes von $y$ in die Gleichung 16) erhält man $\frac{d y}{d t}: \frac{d x}{d t}=\frac{k_{2} A_{1}}{k_{1} A_{2}}$. Das Verhältnis der Reactionsgeschwindigkeiten ist also in diesem Falle wirklich von der Zeit unabhängig. Mit Hilfe des für beliebige $k$-Werte giltigen Wertes von $y$ wïrde man erhalten

$$
\frac{d y}{d t}: \frac{d x}{d t}=\frac{k_{2}}{k_{1}} \frac{A_{1}\left[A_{2}-\left(n_{2}-\alpha_{2}\right) x\right]^{\frac{k_{2}\left(n_{1}+1-\beta_{1}\right)}{k_{1}\left(n_{2}-\alpha_{2}\right)}-1}}{A_{2}^{\frac{k_{2}\left(n_{1}+1-\beta_{1}\right)}{k_{1}\left(n_{2}-\alpha_{3}\right)}}} .
$$

Man sieht sofort, dass die Erfüllung der Gleichung 15 a) für die Constanz des Geschwindigkeitsverhältnisses nothwendig ist.

Specielle Fälle sind beispielsweise

$$
\begin{aligned}
& \left.\begin{array}{ll}
\text { I. } & M_{2} \rightarrow \alpha_{3} M_{3}+\ldots \\
\text { II. } & M_{1} \rightarrow \beta_{3} M_{3}+\ldots
\end{array}\right\} \text { für } k_{1}=k_{2} \\
& \left.\begin{array}{cc}
\text { I. } & M_{1}+M_{2} \rightarrow M_{1}+\alpha_{3} M_{3}+\ldots \\
\text { II. } & 2 M_{1} \rightarrow \beta_{3} M_{3}+\ldots
\end{array}\right\} \text { für } k_{1}=2 k_{2} \text {. }
\end{aligned}
$$

§. 34. Bei Reactionsgleichungen von der im §. 31 angegebenen Form können außerdem constante Geschwindigkeitsverhältnisse bei bestimmten Werten der Anfangsconcentrationen auftreten. Ich beschränke mich darauf, ein Beispiel zu geben.

Es seien die vollständigen Reactionsgleichungen

$$
\begin{array}{ll}
\text { I. } & M_{1}+2 M_{2} \rightarrow \alpha_{3} M_{3}+\alpha_{4} M_{4}+\ldots \\
\text { II. } & 2 M_{1}+M_{2} \rightarrow \beta_{3} M_{3}+\beta_{4} M_{4}+\ldots
\end{array}
$$

Die Gleichung 13) geht über in

$$
0=k_{1} A_{2}\left(2 A_{1}-A_{2}\right)+k_{2} A_{1}\left(A_{1}-2 A_{2}\right) \text {. }
$$

Dieser Gleichung kann u. a. durch $A_{1}=A_{2}, k_{1}=k_{2}$ genügt werden. Man hat 


$$
\frac{d x}{d t}=k(A-x-2 y)(A-2 x-y)^{2}
$$

und

$$
\frac{d y}{d t}=k(A-x-2 y)^{2}(A-2 x-y) .
$$

Durch Integration von $\frac{d y}{d t}: \frac{d x}{d t}=\frac{A-x-2 y}{A-2 x-y}$ erhält mani $l \frac{2 / 3 A-x-y}{(x-y)^{3}}=\infty$, woraus $y=x$ folgt. Die Gleichheit von $x$ und $y$ ergibt sich übrigens auch daraus, dass die Geschwindigkeitsgleichungen durch Vertauschung von $x$ und $y$ keine Veränderung erfahren. Es tritt also auch in diesem Falle ein constantes Geschwindigkeitsverhältnis $\left(\frac{d y}{d t}: \frac{d x}{d t}=1\right)$ auf.

Ein anderes Beispiel ist das zu einem Gleichgewichte führende Reactionspaar $M_{1} \rightarrow M_{2}$ und $M_{2} \rightarrow M_{1}$. Aus Gleichung 13) folgt $k_{1} A_{1}=k_{2} A_{2}$. Man erhält also das in diesem Falle selbstverständliche Resultat, dass ein constantes Geschwindigkeitsverhältnis nur auftritt, wenn die beiden Molekelarten von Anfang an in den dem Gleichgewichte entsprechenden Concentrationen da sind. Dann sind eben die Reactionsgeschwindigkeiten gleich, ihr Verhältnis also eins.

§. 35. Dass die Erfüllung der Gleichung 13) keine hinreichende Bedingung für das Auftreten eines constanten Geschwindigkeitsverhältnisses ist, soll ebenfalls an einem Beispiele gezeigt werden.

Es sollen die Reactionen $2 M_{2} \rightarrow M_{3}$ und $M_{1} \rightarrow M_{4}$ nebeneinander verlaufen.

Dann ist $\frac{d x}{d t}=k_{1}\left(A_{2}-2 x\right)^{2}$ und $\frac{d y}{d t}=k_{2}\left(A_{1}-y\right)$, woraus durch Integration folgt

$$
x=\frac{k_{1} A_{2}^{2} t}{2 k_{1} A_{2} t+1} \quad \text { und } y=A_{1}\left(1-e^{-k_{2} t}\right) .
$$

Daher ist

$$
\frac{d x}{d t}=\frac{k_{1} A_{2}^{2}}{\left(2 k_{1} A_{2} t+1\right)^{2}}, \quad \frac{d y}{d t}=k_{2} A_{1} e^{-k_{2} t}
$$


Die beiden Reactionsgeschwindigkeiten stehen auch dann nicht in einem von der Zeit unabhängigen Verhältnisse, wenn man die durch Gleichung 13) gegebene Bedingung erfüllt.

Diese Bedingung ist im vorliegenden Falle $k_{2}=4 k_{1} A_{2}$. Man erhält

$$
\frac{d y}{d t}: \frac{d x}{d t}=\frac{4 A_{1}}{A_{2}}\left(2 k_{1} A_{2} t+1\right)^{2} e^{-4 k_{1} A_{2} t}
$$

welcher Ausdruck von der Zeit abhängt.

Jedenfalls sind constante Verhältnisse zwischen den Reactionsgeschwindigkeiten in der Regel nur zu erwarten, wenn die beiden Reactionen hinsichtlich der Stoffe von veränderlicher Concentration von gleicher Ordnung sind.

$\S$. 36. Die in $\S .27$ bis 34 besprochenen Fälle von constanten Geschwindigkeitsverhältnissen unterscheiden sich wesentlich von den in $\S .20$ bis 26 behandelten. Bei letzteren ist das Geschwindigkeitsverhältnis gleich dem Verhältnisse der Geschwindigkeitsconstanten, wie in §. 20 erwähnt wurde. Bei ersteren dagegen ist das Geschwindigkeitsverhältnis auch von den Anfangsconcentrationen abhängig. Das ergibt sich unmittelbar aus Gleichung 5) (\$. 16). Zum gleichen Resultate kommt man selbstverständlich auch, wenn man die Gleichung 4) (§. 16) mit Hilfe der Gleichungen 8) (\$. 27) und 9) (\$. 28) umformt.

Aus Gleichung 5) folgt auch, dass das Geschwindigkeitsverhältnis von den Concentrationen der Molekelarten von unveränderlicher Concentration (§.17) abhängt, wenn für diese Molekelarten nicht die Bedingungen $0-n=0$ erfüllt sind.

Man kann daher sagen: Ein von den Anfangsconcentrationen und der Zeit unabhängiges endliches Verhältnis der Geschwindigkeiten zweier nebeneinander verlaufender Reactionen bewerst immer die Gleichheit der linken Seiten der vollständigen Reactionsgleichungen.

§.37. Ein endliches, von der Zeit unabhängiges Verhältnis der Geschwindigkeiten zweier nebeneinander verlaufenden Reactionen tritt auch auf, wenn fiur einen Theil der Stoffe die Bedingung des $\$$. 17, fiur einen anderen Theil die Bedingung des $\$ .20$ (einschlieblich \$. 26 a) und fiir den Rest gruppenweise die Bedingung des $\$ .27$ erfüllt ist. 
Ein einfaches Beispiel dafür sind die vollständigen Reactionsgleichungen

$$
\begin{aligned}
\text { I. } & M_{1}+M_{2}+M_{3} & \rightarrow M_{1}+M_{5} \\
\text { II. } & M_{2}+M_{4} & \rightarrow M_{6} .
\end{aligned}
$$

Der Stoff $M_{1}$ ist von unveränderlicher Concentration, da er nur an der Reaction I, und zwar nur als Katalysator betheiligt ist. Für $M_{2}$ ist die Bedingung $n_{2}=o_{2}$ erfüllt. Ein constantes Geschwindigkeitsverhältnis ist möglich, wenn für $M_{3}$ und $M_{4}$ die Bedingung des $\$ .27$ erfüllt ist. Zur Erfüllung dieser Bedingung ist die Befriedigung der Gleichung 13) erforderlich. Diese gibt im vorliegenden Falle $k_{1} A_{1}=k_{2}$. In der That tritt dann ein von der Zeit unabhängiges Geschwindigkeitsverhältnis auf.

Man hat

$$
\begin{aligned}
& \frac{d x}{d t}=k_{1} A_{1}\left(A_{2}-x-y\right)\left(A_{3}-x\right) \\
& \frac{d y}{d t}=k_{2}\left(A_{2}-x-y\right)\left(A_{4}-y\right) .
\end{aligned}
$$

Daraus ergibt sich

$$
\frac{d x}{d t}: \frac{d y}{d t}=\frac{k_{1} A_{1}\left(A_{3}-x\right)}{k_{2}\left(A_{4}-y\right)}
$$

woraus durch Integration

$$
x=A_{3}\left[1-\left(\frac{A_{4}-y}{A_{4}}\right)^{\frac{k_{1} A_{1}}{k_{2}}}\right]
$$

folgt. Setzt man diesen Wert für $x$ in das Geschwindigkeitsverhältnis ein, so erhält man

$$
\frac{d x}{d t}: \frac{d y}{d t}=\frac{k_{1} A_{1}\left(A_{4}-y\right)^{\frac{k_{3} A_{4}}{k_{z}}}=1}{k_{2} A_{4}^{\frac{k_{i} A_{1}}{k_{2}}}} .
$$

Man sieht, dass nur für $k_{1} A_{1}=k_{2}$ ein constantes Geschwindigkeitsverhältnis auftritt. Sein Wert ist

$$
\frac{d x}{d t}: \frac{d y}{d t}=\frac{k_{1} A_{1}}{k_{2} A_{4}}
$$


$\$$ 38. Geben zwei Reactionen infolge Gleichheit der linken Seiten der vollständigen Reactionsgleichungen (\$.20) ein constantes Geschwindigkeitsverhältnis, so können daneben beliebige andere Reactionen stattfinden, ohne dass die Constanz gestört wird.

Dasselbe gilt auch, wenn an den beiden Reactionen Molekelarten von constanter Concentration betheiligt sind, vorausgesetzt, dass die weiteren Reactionen die Constanz der Concentration nicht aufheben. Das trifft insbesondere zu für Molekelarten, die in großem Überschusse vorhanden sind, ferner für jene, die an allen Reactionen nur als Katalysatoren betheiligt sind.

Im Falle des $\S .27$ dagegen werden die Bedingungen für das Auftreten eines constanten Geschwindigkeitsverhältnisses durch das Hinzutreten weiterer Reactionen, an denen theilweise dieselben Molekelarten betheiligt sind, beeinflusst, wenn diese Reactionen die Concentrationen jener Molekelarten ändern, welche die Geschwindigkeiten der beiden ersten Reactionen beeinflussen.

Beispielsweise gibt das Reactionspaar $M_{1} \rightarrow M_{3}$ und $M_{2} \rightarrow M_{4}$ nach $\S .33$ für $k_{1}=k_{2}$ ein constantes Geschwindigkeitsverhältnis. Findet daneben noch die Reaction $M_{2} \rightarrow M_{5}$ statt, so wird die nothwendige und in diesem Falle auch hinreichende Bedingung für ein constantes Geschwindigkeitsverhältnis zwischen den beiden ersten Reactionen $k_{1}=k_{2}+k_{3}$.

Ist die dritte Reaction $M_{5} \rightarrow M_{2}$, so liefert Gleichung 13) $k_{1}=k_{2}-\frac{A_{5} k_{3}}{A_{2}}$. Diese Bedingung ist aber keine hinreichende Bedingung für das Auftreten eines constanten Geschwindigkeitsverhältnisses zwischen den beiden ersten Reactionen, wie man bei der Integration der Geschwindigkeitsgleichungen sieht.

Aus

$$
\begin{aligned}
& \frac{d x}{d t}=k_{1}\left(A_{1}-x\right) \\
& \frac{d y}{d t}=k_{2}\left(A_{2}-y+z\right) \\
& \frac{d z}{d t}=k_{3}\left(A_{5}-z\right)
\end{aligned}
$$


folgt

$$
\begin{aligned}
& x=A_{1}\left(1-e^{-k_{1} t}\right) \\
& y=A_{2}+A_{5}+\left(\frac{k_{2} A_{5}}{k_{2}-k_{3}}-A_{2}-A_{5}\right) e^{-k_{2} t}-\frac{k_{2} A_{5}}{k_{2}-k_{3}} e^{-k_{3} t} \\
& z=A_{5}\left(1-e^{-k_{3} t}\right) .
\end{aligned}
$$

Daraus ergibt sich

$$
\frac{d y}{d t}: \frac{d x}{d t}=\frac{k_{2}}{k_{1} A_{1}}\left(A_{2}-\frac{k_{3} A_{5}}{k_{2}-k_{3}}\right) e^{\left(k_{1}-k_{2}\right) t}+\frac{k_{2} k_{3} A_{5}}{k_{1}\left(k_{2}-k_{3}\right) A_{1}} e^{\left(k_{1}-k_{3}\right) t} .
$$

Führt man die aus Gleichung 13) folgende Bedingung ein, so erhält man

$$
\begin{gathered}
\frac{d y}{d t}: \frac{d x}{d t}=\frac{k_{2} A_{2}}{A_{1}\left(k_{2} A_{2}-k_{3} A_{5}\right)}\left\{\left(A_{2}-\frac{k_{3} A_{5}}{k_{2}-k_{3}}\right) e^{\frac{-k_{3} A_{5} t}{A_{2}}}+\right. \\
\left.+\frac{k_{3} A_{5}}{k_{2}-k_{3}} e^{\frac{\left(k_{2}-k_{3}\right) A_{2}-k_{3} A_{5}}{A_{3}}}\right\}
\end{gathered}
$$

Dieser Ausdruck ist im allgemeinen nicht constant; die Gleichung 13) liefert daher im vorliegenden Falle keine hinreichende Bedingung für das Auftreten eines constanten Geschwindigkeitsverhältnisses. Wohl aber wird der Ausdruck constant in dem speciellen Falle $\left(k_{2}-k_{3}\right) A_{2}-k_{3} A_{5}=0$. Die nothwendige und hinreichende Bedingung für das Auftreten eines constanten Geschwindigkeitsverhältnisses zwischen den beiden ersten Reactionen ist daher

$$
k_{3} A_{\tilde{z}}=\left(k_{2}-k_{1}\right) A_{2}=\left(k_{2}-k_{3}\right) A_{2},
$$

woraus $k_{1}=k_{3}$ folgt.

Man erhält dann

$$
\frac{d y}{d t}: \frac{d x}{d t}=\frac{k_{2} A_{2}}{k_{3} A_{1}}
$$

Für $k_{2}=k_{3}$ wird die allgemeine Formel für $y$ unbestimmt. In diesem Falle hat man 


$$
\begin{gathered}
y=A_{2}+A_{5}-\left(A_{2}+A_{5}+k_{2} A_{5} t\right) e^{-k_{2} t} \\
\frac{d y}{d t}: \frac{d x}{d t}=\frac{k_{2}\left(A_{2}+k_{2} A_{5} t\right)}{k_{1} A_{1}} e^{\left(k_{1}-k_{3}\right) t}
\end{gathered}
$$

Letzterer Ausdruck kann für endliche $k$ und $A$ nicht constant werden. Dasselbe folgt aus Gleichung $16 a$ ). Diese führt für $k_{2}=k_{3}$ zu $A_{5}=0$, also zum Verschwinden der dritten Reaction.

$\$ 38 \mathrm{a}$. Von besonderer Wichtigkeit sind die Reactionen, an welchen Elektrolyte betheiligt sind. In solchen Fällen hat man neben den sonstigen Reactionen immer die Reactionspaare, welche zum Gleichgewichte fiuhren. Ich will daher noch zwei vollständig durchgerechnete Beispiele geben, in welchen neben den beiden Reactionen, die auf constantes Geschwindigkeitsverhältnis untersucht werden sollen, noch ein $\mathrm{zu}$ einem Gleichgewichte führendes Reactionspaar angenommen wird.

Es seien zunächst die stattfindenden Reactionen I. $M_{1} \rightarrow M_{3}$ II. $M_{2} \rightarrow M_{4}$ III. $M_{1} \rightarrow M_{2}$ IV. $M_{2} \rightarrow M_{1}$. Die Veränderlichen der vier Reactionen seien $x, y, z, u$. Als nothwendige Bedingung für das Auftreten eines constanten Geschwindigkeitsverhältnisses zwischen den Reactionen I und II folgt aus Gleichung 13)

$$
0=k_{1}-k_{2}+k_{3}\left(1+\frac{A_{1}}{A_{2}}\right)-k_{4}\left(1+\frac{A_{2}}{A_{1}}\right) \text {. }
$$

Setzt $\operatorname{man} z-u=v$, so sind die Geschwindigkeitsgleichungen

$$
\begin{aligned}
& \frac{d x}{d t}=k_{1}\left(A_{1}-x-z+u\right)=k_{1}\left(A_{1}-x-v\right) \\
& \frac{d y}{d t}=k_{2}\left(A_{2}-y+z-u\right)=k_{2}\left(A_{1}-y+v\right) \\
& \frac{d v}{d t}=\frac{d z}{d t}-\frac{d u}{d t}=k_{3}\left(A_{1}-x-v\right)-k_{4}\left(A_{2}-y+v\right)
\end{aligned}
$$

Setzt man

$$
\begin{aligned}
\Sigma k & =k_{1}+k_{2}+k_{3}+k_{4} \\
S & =k_{1} k_{2}+k_{1} k_{4}+k_{2} k_{3} \\
b & =+\sqrt{\frac{(\Sigma k)^{2}}{4}-k_{1} k_{2}-k_{1} k_{4}-k_{2} k_{3}}
\end{aligned}
$$




$$
\begin{aligned}
& \vartheta_{1}=\frac{\Sigma k}{2}+\sqrt{\frac{\left(\sum k\right)^{2}}{4}-k_{1} k_{2}-k_{1} k_{4}-k_{2} k_{3}} \\
& \vartheta_{2}=\frac{\Sigma k}{2}-\sqrt{\frac{(\Sigma k)^{2}}{4}-k_{1} k_{2}-k_{1} k_{4}-k_{2} k_{3}} \\
& T=\left(k_{2}+k_{4}\right) A_{1}+k_{4} A_{2},
\end{aligned}
$$

so sind die Integrale der Differentialgleichungen

$$
\begin{gathered}
x=-\left(1-e^{-\vartheta_{1} t}\right) \frac{\left(T-A_{1} \vartheta_{1}\right) k_{1} \vartheta_{2}}{2 b S}+\left(1-e^{-\vartheta_{2} t}\right) \frac{\left(T-A_{1} \vartheta_{2}\right) k_{1} \vartheta_{1}}{2 b S} \\
y=\frac{k_{2}}{2 b k_{4} S}\left\{\left(1-e^{-\vartheta_{1} t}\right)\left(T-A_{1} \vartheta_{1}\right)\left[S-\left(k_{1}+k_{3}\right) \vartheta_{2}\right]+\right. \\
\left.+\left(1-e^{-\vartheta_{2} t}\right)\left(T-A_{1} \vartheta_{2}\right)\left[\left(k_{1}+k_{3}\right) \vartheta_{1}-S\right]\right\} \\
v=\frac{1}{2 b S}\left\{-\left(1-e^{-\vartheta_{1} t}\right)\left(T-A_{1} \vartheta_{1}\right)\left(S-k_{1} \vartheta_{2}\right)+\right. \\
\left.+\left(1-e^{-\vartheta_{2} t}\right)\left(T-A_{1} \vartheta_{2}\right)\left(S-k_{1} \vartheta_{1}\right)\right\} .
\end{gathered}
$$

Hieraus folgt

$$
\begin{aligned}
& \frac{d x}{d t}=\frac{k_{1}}{2 b}\left\{-\left(T-A_{1} \vartheta_{1}\right) e^{-\vartheta_{1} t}+\left(T-A_{1} \vartheta_{2}\right) e^{-\vartheta_{2} t}\right\} \\
& \frac{d y}{d t}=-\frac{k_{2}}{2 b k_{4}}\left\{\left(\dot{T}-A_{1} \vartheta_{1}\right)\left(k_{2}+k_{4}-\vartheta_{2}\right) e^{-\vartheta_{1} t}-\right. \\
& \left.-\left(T-A_{1} \vartheta_{2}\right)\left(k_{2}+k_{4}-\vartheta_{1}\right) e^{-\vartheta_{2} t}\right\} \\
& \frac{d x}{d t}: \frac{d y}{d t}= \\
& =\frac{k_{1} k_{4}}{k_{2}} \cdot \frac{-\left(T-A_{1} \vartheta_{1}\right)+\left(T-A_{1} \vartheta_{2}\right) e^{2 b t}}{\left(T-A_{1} \vartheta_{1}\right)\left(k_{2}+k_{4}-\vartheta_{2}\right)-\left(T-A_{1} \vartheta_{2}\right)\left(k_{2}+k_{4}-\vartheta_{1}\right) e^{2 b t}} .
\end{aligned}
$$

Letzteren Ausdruck kann man auf eine etwas andere Form bringen, indem man Zähler und Nenner mit $e^{-2 b t}$ multipliciert.

Der aufgeschriebene Ausdruck wird von der Zeit unabhängig, wenn $T-A_{1} \vartheta_{2}=0$. Aus dem durch Multiplication des Zählers und Nenners, mit $e^{-2 b t}$ umgeformten Ausdrucke ergibt sich ferner, dass er auch für $T-A_{1} \vartheta_{1}=0$ constant wird. Diese beiden Bedingungen für die Constanz des. Geschwindigkeitsverhältnisses lassen sich auf die Form bringen 


$$
\frac{A_{2}}{A_{1}}=\frac{1}{k_{4}}\left(\frac{\Sigma k}{2}-k_{2}-k_{4} \mp b\right) \text {. }
$$

Denselben Ausdruck erhält man, wenn man Gleichung 16b) nach $\frac{A_{2}}{A_{1}}$ auflöst. Die aus Gleichung 13 ) folgende Bedingung für das Auftreten eines constanten Geschwindigkeitsverhältnisses ist daher im vorliegenden Falle nicht bloß eine nothwendige, sondern auch eine hinreichende.

Eine besonders einfache Art, die Gleichung $16 b$ ) zu befriedigen, ist gegeben durch $k_{1}=k_{2}, k_{3}=k_{4}, A_{1}=A_{2}$. Dann ist $x=y=A_{1}\left(1-e^{-k_{1} t}\right), v=0$.

§. 38b. Verlaufen die Reactionen III und IV mit unendlich großer Geschwindigkeit, so liegt der Fall des \$. $26 a$ vor; die linken Seiten der Reactionsgleichungen I und II sind als identisch $\mathrm{zu}$ betrachten. Sie enthalten nämlich hylotrope Formen, zwischen denen sich unendlich rasch ein Gleichgewicht herstellt. In diesem Falle muss nach $\$ .26 a$ zwischen den Reactionen I und II für beliebige Werte der Geschwindigkeitsconstanten und Anfangsconcentrationen ein von der Zeit unabhängiges Geschwindigkeitsverhältnis auftreten.

Dass dies wirklich der Fall ist, sieht man, wenn man in der im vorigen Paragraphen gegebenen allgemeinen Gleichung für $\frac{d x}{d t}: \frac{d y}{d t} k_{3}$ und $k_{4}$ gleich unendlich setzt. Bezeichnet man die Gleichgewichtsconstante $\frac{k_{3}}{k_{4}}$ mit $x$, so wird

$$
\begin{aligned}
\Sigma k & =k_{4}(x+1) \\
S & =k_{4}\left(k_{1}+k_{2} x\right) \\
b & =\frac{k_{4}(x+1)}{2} \\
\vartheta_{1} & =k_{4}(x+1) \\
\vartheta_{2} & =\frac{k_{1}+k_{2} \dot{x}}{x+1}
\end{aligned}
$$

Dadurch wird

$$
\frac{d x}{d t}: \frac{d y}{d t}=\frac{k_{1}}{k_{2}^{\prime}} \cdot \frac{x A_{1}-A_{2}+\left(A_{1}+A_{2}\right) e^{k_{4}(x+1) t}}{A_{2}-\% A_{1}+x\left(A_{1}+A_{2}\right) \cdot e^{k_{4}(x+1) t}}=\frac{\infty}{\infty} .
$$


Bestimmt man den Wert dieses Ausdruckes durch Differentiation oder berücksichtigt man, dass $x A_{1}=A_{2}$, weil das Gleichgewicht sich unendlich rasch einstellt, so erhält man

$$
\frac{d x}{d t}: \frac{d y}{d t}=\frac{k_{1}}{k_{2} x} .
$$

Gleichung 16b) gibt in diesem Falle die selbstverständliche Bedingung $\times A_{1}=A_{2}$.

$\S 38 c$. Es sollen nunmehr folgende Reactionen betrachtet werden:

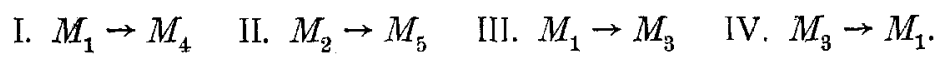

Die Veränderlichen der vier Reactionen seien wieder $x, y, z, u$.

Als nothwendige Bedingung für das Auftreten eines constanten Geschwindigkeitsverhältnisses zwischen den Reactionen I und II ergibt sich aus Gleichung 13)

$$
0=k_{1}-k_{2}+k_{3}-k_{4} \frac{A_{3}}{A_{1}} .
$$

Die Geschwindigkeitsgleichungen sind

$$
\begin{aligned}
& \frac{d x}{d t}=k_{1}\left(A_{1}-x-v\right) \\
& \frac{d y}{d t}=k_{2}\left(A_{2}-y\right) \\
& \frac{d v}{d t}=k_{3}\left(A_{1}-x-v\right)-k_{4}\left(A_{3}+v\right),
\end{aligned}
$$

wo $v=z-u$.

Führt man folgende Bezeichnungen ein:

$$
\begin{aligned}
\Sigma k & =k_{1}+k_{3}+k_{4} \\
b & =+\sqrt{\frac{(\Sigma k)^{2}}{4}-k_{1} k_{4}} \\
\vartheta & =\frac{\Sigma k}{2}+\sqrt{\frac{(\Sigma k)^{2}}{4}-k_{1} k_{4}}
\end{aligned}
$$




$$
\begin{aligned}
\vartheta^{\prime} & =\frac{\Sigma k}{2}-\sqrt{\frac{(\Sigma k)^{2}}{4}-k_{1} k_{4}} \\
\lambda & =\frac{k_{1}}{\vartheta-k_{3}-k_{4}} \\
\lambda^{\prime} & =\frac{k_{1}}{\vartheta^{\prime}-k_{3}-k_{4}},
\end{aligned}
$$

so sind die Integrale der Differentialgleichungen

$$
\begin{aligned}
& x=\frac{k_{1} k_{3}}{2 b}\left\{-\frac{k_{1} A_{1}+\lambda\left(k_{3} A_{1}-k_{4} A_{3}\right)}{\vartheta\left(\vartheta^{\prime}-k_{3}-k_{4}\right)}\left(1-e^{-\vartheta t}\right)+\right. \\
& \left.+\frac{k_{1} A_{1}+\lambda^{\prime}\left(k_{3} A_{1}-k_{4} A_{3}\right)}{\vartheta^{\prime}\left(\vartheta-k_{3}-k_{4}\right)}\left(1-e^{-\vartheta^{\prime} t}\right)\right\} \\
& y=A_{2}\left(1-e^{-k_{2} t}\right) \\
& v=\frac{k_{3}}{2 b}\left\{\frac{1}{q}\left[k_{1} A_{1}+\lambda\left(k_{3} A_{1}-k_{4} A_{3}\right)\right]\left(1-e^{-\vartheta t}\right)-\right. \\
& \left.-\frac{1}{\vartheta^{\prime}}\left[k_{1} A_{1}+\lambda^{\prime}\left(k_{3} A_{1}-k_{4} A_{3}\right)\right]\left(1-e^{-\vartheta^{\prime} t}\right)\right\} .
\end{aligned}
$$

Hieraus folgt

$$
\begin{aligned}
\frac{d x}{d t}=\frac{k_{1} k_{3}}{2 b}\left\{-\frac{k_{1} A_{1}+\lambda\left(k_{3} A_{1}-k_{4} A_{3}\right)}{\vartheta^{\prime}-k_{3}-k_{4}} e^{-\vartheta t}+\right. & \left.+\frac{k_{1} A_{1}+\lambda^{\prime}\left(k_{3} A_{1}-k_{4} A_{3}\right)}{\vartheta-k_{3}-k_{4}} e^{-\vartheta^{\prime} t}\right\} \\
\frac{d y}{d t}= & k_{2} A_{2} e^{-k_{2} t}
\end{aligned}
$$

$$
\begin{aligned}
\frac{d x}{d t}: \frac{d y}{d t}=\frac{k_{1}}{2 b k_{2} A_{2}}\left\{\left[A_{1}\left(\vartheta-k_{4}\right)-\right.\right. & \left.-k_{4} A_{3}\right] e^{\left(k_{2}-\vartheta\right) t}- \\
& \left.-\left[A_{1}\left(\vartheta^{\prime}-k_{4}\right)-k_{4} A_{3}\right] e^{\left(k_{2}-\vartheta^{\prime}\right) t}\right\} .
\end{aligned}
$$

Dieser Ausdruck wird von der Zeit unabhängig für $k_{2}=\vartheta$ und $A_{1}\left(\vartheta^{\prime}-k_{4}\right)=k_{4} A_{3}$ oder für $k_{2}=\vartheta^{\prime}$ und $A_{1}\left(\vartheta-k_{4}\right)=k_{4} A_{3}$. Durch Einsetzung der. Werte für $\vartheta$ und $\vartheta^{\prime}$ gehen diese zwei Paare von Bedingungen über in 


$$
k_{2}=\frac{\Sigma k}{2} \pm b
$$

Man sieht, dass diese Bedingungen die Gleichung 16c) befriedigen, wie es sein muss. Aber diese Gleichung hat auch noch andere Lösungen, welche den Ausdruck für $\frac{d x}{d t}: \frac{d y}{d t}$ nicht constant machen; sie ist also im vorliegenden Falle keine hinreichende Bedingung für die Unabhängigkeit des Geschwindigkeitsverhältnisses von der Zeit.

§. 38d. Nimmt man nun wieder an, dass die Reactionen III und IV mit unendlicher Geschwindigkeit verlaufen, so ist $k_{3}=\infty, k_{4}=\infty$. Setzt $\operatorname{man} \frac{k_{3}}{k_{4}}=x$, so wird

$$
b=\frac{k_{4}(x+1)}{2}, \quad \vartheta=k_{4}(x+1), \quad \vartheta^{\prime}=\frac{k_{1}}{x+1} .
$$

Man erhält daher

$$
\frac{d x}{d t}: \frac{d y}{d t}=\frac{k_{1}\left(A_{1}+A_{3}\right)}{k_{2} A_{2}(x+1)} e^{\left(k_{2}-\frac{k_{1}}{x+1}\right) t}=\frac{k_{1} A_{1}}{k_{2} A_{2}} e^{\left(k_{2}-\frac{k_{1}}{x+1}\right) t},
$$

da $A_{3}=\tilde{x} A_{1}$ ist.

Das Geschwindigkeitsverhältnis erhält im betrachteten Falle einen endlichen constanten Wert, wenn $k_{2}=\frac{k_{1}}{x+1}$. Das folgt auch aus den durch Gleichung 16d) ausgedrückten Bedingungen. Denn diese gehen für $k_{3}=k_{4}=\infty$ über in

$$
k_{2}=\infty, \quad \frac{A_{3}}{A_{1}}=-1
$$

oder

$$
k_{2}=\frac{k_{1}}{x+1}, \quad \frac{A_{3}}{A_{1}}=x
$$


Das erste Paar von Bedingungen hat keinen chemischen Sinn, da die Anfangsconcentrationen wesentlich positive Größen sind, also $\frac{A_{3}}{A_{1}}=-1$ unmöglich jst. Das zweite Paar von Bedingungen entspricht der Folgerung, die sich aus der Formel für das Geschwindigkeitsverhältnis ergibt; denn $\frac{A_{3}}{A_{1}}=x$ ist selbstverständlich, wenn $k_{3}$ und $k_{4}$ unendlich sind.

Die aus Gleichung 13) folgende Bedingung 16c) versagt für unendliche $k_{3,}$ und $k_{4}$, da sie dann nur zur selbstverständlichen Gleichgewichtsbedinguing $x=\frac{A_{3}}{A_{1}}$ führt.

§. 38e. Ähnlich wie im zuletzt besprochenen Falle versagt die Gleichung 13) immer, wenn unendlich rasch sich einstellende Gleichgewichte zu den in die Gleichung eintretenden Reactionen gehören. Man kann jedoch von der Gleichung 13) Gebrauch machen, wenn man entsprechend $8.6 b$ die Gleichgewichtsbedingungen in die Geschwindigkeitsgleichungen der langsamer verlaufenden Reactionen einführt.

In dem im vorigen Paragraphen besprochenen Beispiele kann man die Reactionsgleichungen III und IV ersetzen durch die Gleichgewichtsbedingung $x\left(A_{1}-\xi_{1}\right)=A_{3}-\xi_{3}$. Nun sei $A_{1}+A_{3}=A$, wo $A$ die Anfangsconcentration der gesammten hylotropen Gruppe bedeutet. Nach $\S .5$ ist ferner $\xi_{1}+\xi_{3}=x$, da $\xi_{1}+\xi_{3}$ die Änderung der Gesammtconcentration der hylotropen Gruppe bedeutet, welche von den Reactionen mit Gegenwirkungen nicht beeinflusst wird. Daher ist

$$
A_{1}-\xi_{1}+A_{3}-\xi_{3}=A-x .
$$

Durch Einführung der Gleichgewichtsbedingung folgt daraus

$$
A_{1}-\dot{\xi}_{1}=\frac{A-x}{x+1} .
$$

Die Geschwindigkeitsgleichungen gehen über in

$$
\frac{d x}{d t}=\frac{k_{1}}{x+1}(A-x) \text { und } \frac{d y}{d t}=k_{2}\left(A_{2}-y\right) \text {. }
$$


Beim Stattfinden der Reactionen I bis IV und unendlichen $k_{3}$ und $k_{4}$ haben daher $\frac{d x}{d t}$ und $\frac{d y}{d t}$ dieselben Werte, wie wenn lediglich die Reactionen $M \rightarrow M_{4}$ und $M_{2} \rightarrow M_{5}$, und $z$ war mit den Geschwindigkeitsconstanten $\frac{k_{1}}{x+1}$ und $k_{2}$ eintreten. $M$ bedeutet darin eine einheitliche Molekelart, durch die man sich die hylotrope Gruppe $M_{1} \rightleftarrows M_{3}$ ersetzt denkt.

Auf dieses fingierte Reactionspaar kann nunmehr die Gleichung 13) angewendet werden. Man erhält so als nothwendige und hinreichende (vergl. §. 33) Bedingung für das Auftreten eines constanten Geschwindigkeitsverhältnisses $\frac{k_{1}}{x+1}=$ $=k_{2}$, also dieselbe Bedingung, welche sich im vorigen Paragraphen aus der Integration der den Reactionen I bis IV entsprechenden Differentialgleichungen ergeben hat.

Im Beispiele des $\S .38 b$ ) hat man $x\left(A_{1}-\xi_{1}\right)=A_{2}-\xi_{2}$, $\xi_{1}+\xi_{2}=x+y, A_{1}-\xi_{1}+A_{2}-\xi_{2}=A-x-y$. Daraus folgt

$$
A_{1}-\xi_{1}=\frac{1}{x+1}(A-x-y), \quad A_{2}-\xi_{2}=\frac{x}{x+1}(A-x-y) .
$$

Die Geschwindigkeitsgleichungen sind daher

$$
\frac{d x}{d t}=\frac{k_{1}}{x+1}(A-x-y), \quad \frac{d y}{d t}=\frac{k_{2} x}{x+1}(A-x-y),
$$

woraus die nicht an besondere Bedingungen geknüpfte Constanz des Greschwindigkeitsverhältnisses ersichtlich ist.

Die Anwendung der Gleichung 13) ergibt in diesem Falle eine identisch befriedigte Gleichung. Denn den umgeformten Geschwindigkeitsgleichungen entsprechen die fingierten Reactionen $M \rightarrow M_{4}$ und $M \rightarrow M_{5}$ mit den Geschwindigkeitsconstanten $\frac{k_{1}}{x+1}$ und $\frac{k_{2} x}{x+1}$. Für diese fingierten Reactionen wird $n_{1}-o_{1}=0$; da nur ein Stoff $z u$ berücksichtigen ist, werden also beide Seiten der Gleichung 13) Null. 


\section{Constante Concentrationen.}

§. 39. Im §. 17 ist bereits erwähnt worden, dass eine Molekelart unter Umständen eine von der Zeit unabhängige Concentration haben kann, wenn sie bei einem Theile der nebeneinander verlaufenden Reactionen gebildet, bei einem anderen Theile verbraucht wird.

Die Bedingungen, unter welchen das eintritt, ergeben sich aus folgendem.

§. 40. Es soll die Concentration der Molekelart $M_{1}$ unveränderlich sein, obwohl sie an mehreren Reactionen derart betheiligt ist, dass die einzelnen Reactionen ihre Concentration verändern.

Dann muss für diese Molekelart $\xi_{1}(\S .4)$ unabhängig von der Zeit gleich Null sein. Nach Formel 2) ist daher

$$
0=\left(n_{1}-\alpha_{1}\right) x+\left(o_{1}-\beta_{1}\right) y+\ldots
$$

Diese Gleichung eignet sich nicht wohl zur Besprechung, da die $x, y \ldots$ erst durch Integration der Geschwindigkeitsgleichungen als Functionen der Zeit dargestellt werden können. Es muss aber auch $\frac{d \xi_{1}}{d t}=0$ sein. Man erhält daher

$$
0=\left(n_{1}-\alpha_{1}\right) \frac{d x}{d t}+\left(o_{1}-\beta_{1}\right) \frac{d y}{d t}+\ldots
$$

Diese Gleichung kann durch $\left(n_{1}-\alpha_{1}\right)=\left(o_{1}-\beta_{1}\right)=\ldots=0$ befriedigt werden. Dann wirkt der Stoff $M_{1}$ nur als Katalysator; die stattfindenden Reactionen haben auf seine Concentration keinen Einfluss. Dieser Fall kommt hier nicht in Betracht.

§. 41. Es können aber auch die Gleichungen $n_{1}=\alpha_{1}$ u. s.w. nur für einen Theil der Reactionen oder für gar keine erfüllt sein. Dann sind Reactionen vorhanden, welche die Concentration der Molekelart $M_{1}$ ändern. Damit die Concentration von $M_{1}$ trotzdem unveränderlich bleibt, muss die Gleichung 17) für jedes $t$ erfüllt sein. Das trifft $z u$, wenn in den einzelnen Summanden dieselbe Zeitfunction vorkommt und nach Heraushebung dieser Function der Factor Null übrig bleibt. 
Die Bedingung, dass in den einzelnen Summanden dieselbe Zeitfunction vorkommt, wird wiedergegeben durch die Gleichung

$$
\frac{d x}{d t}=K_{2} \frac{d y}{d t}=K_{3} \frac{d z}{d t}=\ldots
$$

wo die $K_{2}, K_{3}, \ldots$ von der Zeit unabhängig sind. Es müssen also die Geschwindigkeiten jener Reactionen, welche die Concentration der Molekelart $M_{1}$ verändern, in einem von der Zeit unabhängigen Verhältnisse stehen. Unter welchen Umständen das der Fall ist, wurde im Abschnitte IV besprochen.

Führt man diese Bedingung in die Gleichüng 17) ein, so erhält manı

$$
0=\left(n_{1}-\alpha_{1}\right)+\frac{o_{1}-\beta_{1}}{K_{2}}+\frac{p_{1}-n_{1}}{K_{3}}+\ldots
$$

Setzt man die Werte der $K$ nach Gleichung 5) (\$.16) ein, so folgt

$$
\begin{aligned}
0=\left(n_{1}-\alpha_{1}\right) k_{1} A_{1}^{n_{1}} A_{2}^{n_{2}} \ldots+ & \left(o_{1}-\beta_{1}\right) k_{2} A_{1}^{n_{1}} A_{2}^{o_{2}} \ldots+ \\
& +\left(p_{1}-\gamma_{1}\right) k_{3} A_{1}^{p_{1}} A_{2}^{p_{2}} \ldots+\ldots
\end{aligned}
$$

Die Concentration einer Molekelart ist also unveränderlich, obwohl sie von einzelnen der nebeneinander verlaufenden Reactionen verändert wird, wenn folgende zwei Bedingungen erfüllt sind:

1. Zwischen jenen Reactionen, welche die Concentration der betreffenden Molekelart verändern, muss ein constantes Geschwindigkeitsverhältnis (entsprechend dem Abschnitte IV) bestehen.

2. Es muss die Gleichung 18) erfüllt sein, welche eine Beziehung zwischen den auf diese Molekelart bezüglichen Coefficienten in den Reactionsgleichungen, ferner den Geschwindigkeitscoefficienten jener Reactionen, welche die Concentration dieser Molekelart verändern, und den in den Geschwindigkeitsgleichungen dieser Reactionen auftretenden Anfangsconcentrationen ausdrückt.

§. 42. Es soll zunächst folgendes Beispiel untersucht werden. Zwischen den Reactionen 
und

$$
n_{1} M_{1}+n_{2} M_{2} \rightarrow \alpha_{1} M_{1}+\alpha_{2} M_{2}+\ldots
$$

$$
o_{1} M_{1}+n_{2} M_{2} \rightarrow \beta_{1} M_{1}+\beta_{1} M_{2}+\ldots
$$

tritt gemäß §. 20 ein constantes Geschwindigkeitsverhältnis auf, wenn die Concentration von $M_{1}$ unveränderlich ist, da hinsichtlich der Stoffe von veränderlicher Concentration (in diesem Falle $M_{2}$ ) Gleichheit der linken Seiten der vollständigen Reactionsgleichungen besteht.

Es soll nun untersucht werden, unter welchen Umständen die Concentration von $M_{1}$ unveränderlich ist, wenn außer diesen beiden Reactionen nur solche Reactionen stattfinden, welche die Menge von $M_{1}$ nicht verändern.

Als Bedingung dafür folgt aus Gleichung 18)

$$
0=\left(n_{1}-\alpha_{1}\right) k_{1} A_{1}^{n_{1}}+\left(o_{1}-\beta_{1}\right) k_{2} A_{1}^{o_{1}},
$$

da $A_{2}^{n_{2}}$ herausfällt. Anders geschrieben:

$$
n_{1}-\alpha_{1}=\left(\beta_{1}-o_{1}\right) \frac{k_{2}}{k_{1}} A_{1}^{o_{1}-n_{1}} .
$$

Diese Bedingung kann (was übrigens selbstverständlich ist) nur erfüllt werden, wenn $n_{1}-\alpha_{1}$ und $o_{1}-\beta_{1}$ verschiedenes Zeichen haben, wenn also $M_{1}$ bei der einen Reaction gebildet, bei der anderen verbraucht wird. Außerdem müssen $n_{1}-a_{1}$ und $o_{1}-\beta_{1}$ ganze Zahlen sein.

Ein einfacher specieller Fall wird dargestellt durch die Reactionsgleichungen $M_{1}+2 M_{2} \rightarrow M_{3}$ und $2 M_{2} \rightarrow M_{1}+M_{4}$ für $k_{2}=k_{1}$ und $A_{1}=1$. Diese Reactionsgleichungen geben

$$
\frac{d x}{d t}=k_{1}\left(A_{1}-x+y\right)\left(A_{2}-2 x-2 y\right)^{2} .
$$

und

$$
\frac{d y}{d t}=k_{2}\left(A_{2}-2 x-2 y\right)^{2}
$$

woraus folgt

$$
x=\left(\frac{k_{2}}{k_{1}}-A_{1}\right)\left(e^{\frac{-k_{1}}{k_{3}} y}-1\right)+y
$$


Bildet man nun $\xi_{1}=x-y$ und setzt die Werte $k_{1}=k_{2}$ und $A_{1}=1$ ein, so erhält man $\xi_{1}=0$.

Dieses Reactionspaar stellt hinsichtlich $M_{1}$ eine Folgewirkung dar; es ergibt sich also das interessante Resultat, dass es Folgewirkungen gibt, bei denen das $Z$ wischenproduct von unveränderlicher Concentration ist.

§. 43. Die durch die Gleichung 19) dargestellte Bedingung wird von den Anfangsconcentrationen völlig unabhängig, wenn $n_{1}=o_{1}$ ist.

Das ist der Fall bei den Reactionsgleichungen $M_{1}+M_{2} \rightarrow M_{3}$ und $M_{1}+M_{2} \rightarrow 2 M_{1}+M_{4}$. Beim Stattfinden dieses Reactionspaares hat $M_{1}$ eine unveränderliche Concentration, wenn $k_{1}=k_{2}$, da dann Gleichung 19) erfüllt ist. Man sieht sofort, dass

$$
\frac{d \xi_{1}}{d t}=\frac{d x}{d t}-\frac{d y}{d t}=0
$$

ist.

§. 44. Ein Beispiel, bei dem die Constanz der Geschwindigkeitsverhältnisse nach $\S .27$ ff. eintritt, ist folgendes. Die Reactionsgleichungen seien

$$
n_{1} M_{1}+M_{2} \rightarrow \alpha_{1} M_{1}+M_{4} \text { und } o_{1} M_{1}+M_{3} \rightarrow \beta_{1} M_{1}+M_{5} .
$$

Die Geschwindigkeiten dieser beiden Reactionen können im allgemeinen nur dann in einem constanten Verhältnisse stehen, wenn gemäß Gleichung 13) (§. 30) die Bedingung erfüllt ist

$$
\begin{array}{r}
0=\left(n_{1}-o_{1}\right)\left[\left(n_{1}-\alpha_{1}\right) k_{1} A_{1}^{n_{1}-1} A_{2}+\left(o_{1}-\beta_{1}\right) k_{2} A_{1}^{o_{1}-1} A_{3}\right]+ \\
+ \\
+k_{1} A_{1}^{n_{1}-k_{2} A_{1}^{o_{1}} .}
\end{array}
$$

Ist jedoch die Molekelart $M_{1}$ von unveränderlicher Concentration, so ist sie bei Anwendung der Gleichung 13) nicht zu berücksichtigen; es bleibt daher

$$
k_{1} A_{1}^{n_{1}}=k_{2} A_{1}^{o_{1}} \ldots
$$

Dưrch Integration der Geschwindigkeitsgleichungen überzeugt man sich leicht, dass unterVoraussetzung unveränderlicher Concentrationen von $M_{1}$ und bei Erfüllung der Gleichung 21) ein constantes Geschwindigkeitsverhältnis auftritt. 
Damit aber $M_{1}$ von unveränderlicher Concentration sei, ist gemäß Gleichung 18) (\$.41) erforderlich, dass

sei.

$$
0=\left(n_{1}-\alpha_{1}\right) k_{1} A_{1}^{n_{1}} A_{2}+\left(o_{1}-\beta_{1}\right) k_{2} A_{1}^{o_{1}} A_{3}
$$

(Man sieht, dass die Einführung dieser Bedingung in Gleichung 20) das erste Glied zum Verschwinden bringt. Der Fall des constanten Geschwindigkeitsverhältnisses bei unveränderlicher Concentration von $M_{1}$ ist daher ein specieller Fall des constanten Geschwindigkeitsverhältnisses bei veränderlicher Concentration von $M_{1}$.)

Durch Einführung von 21) in die letzte Gleichung erhält man $\left(n_{1}-\alpha_{1}\right) A_{2}=\left(\beta_{1}-o_{1}\right) A_{3}$. Ist diese Bedingung und die Gleichung 21) erfüllt, so ist die Concentration von $M_{1}$ constant.

$\$$. 45. Das trifft unter anderem $z u$, wenn man setzt $A_{2}=A_{3}$, $\alpha_{1}=o_{1}=0, n_{1}=\beta_{1}=1$. Die Reactionsgleichungen sind dann $M_{1}+M_{2} \rightarrow M_{4}$ und $M_{3} \rightarrow M_{1}+M_{5}$. Bei diesem Reactionspaare ist also die Concentration von $M_{1}$ unveränderlich, wenn $A_{2}=A_{3}$ und (gemäß Gleichung 21) $k_{1} A_{1}=k_{2}$. Man kann sich davon durch die allerdings etwas beschwerliche Integration der Geschwindigkeitsgleichungen überzeugen.

Man hat für dieses Reactionspaar im allgemeinen

$$
\frac{d x}{d t}=k\left(A_{1}-x+y\right)\left(A_{2}-x\right) \text { und } \frac{d y}{d t}=k_{2}\left(A_{3}-y\right) \text {. }
$$

Aus letzterer Differentialgleichung folgt $y=A_{3}\left(1-e^{-k_{3}}\right)$. Entwickelt man die Exponentialfunction in eine Reihe, so erhält man

$$
y=A_{3}\left(k_{2} t-\frac{k_{2}^{2} t^{2}}{2 !}+\frac{k_{2}^{3} t^{3}}{3 !}-\ldots\right)
$$

Setzt man diesen Wert in die Gleichung für $\frac{d x}{d t}$ ein, so kann man die Integration durch Reihen ausführen.

Führt man die Bedingungen $A_{2}=A_{3}$ und $k_{1} A_{1}=k_{2}$ ein, so erhält man

$$
y=A_{2}\left(k_{1} A_{1} t-\frac{k_{1}^{2} A_{1}^{2} t^{2}}{2 !}+\frac{k_{1}^{3} A_{3}^{3} t^{3}}{3 !}-\ldots\right)
$$


und

$$
x=N_{1} t+N_{2} t^{2}+N_{3} t^{3}+\ldots
$$

wobei die Methode der unbestimmten Coefficienten für $N_{1}, N_{2} \ldots$ folgende Werte liefert:

$$
\begin{aligned}
& N_{1}=k_{1} A_{1} A_{2} \\
& N_{2}=-\frac{k_{1}^{2} A_{1}^{2} A_{2}}{2} \\
& N_{3}=\frac{k_{1}^{3} A_{1}^{3} A_{2}}{6} \\
& N_{m+1}=\frac{k_{1}}{m+1}\left[N_{m-1} N_{1}+N_{m-2} N_{2}+\ldots+N_{1} N_{m-1}-\left(A_{1}+A_{2}\right) N_{m}^{r}\right. \\
& -k_{1} A_{1} A_{2} N_{m-1}+\frac{k_{1}^{2} A_{1}^{2} A_{2} N_{m-2}}{2 !}-\cdots^{2}+(-1)^{m-1} \frac{k_{1}^{n-1} A_{1}^{m-1} A_{2} N_{1}}{(n-1) !} \\
& \left.+(-1)^{n-1} \frac{k_{1}^{n} A_{1}^{n} A_{2}^{2}}{\therefore n !}\right]
\end{aligned}
$$

Indem man die ersten $N$ ausrechnet, sieht man bald, dass sie nach der einfachen Gleichung $N_{m+1}=-\frac{k_{1} A_{1}}{m+1} N_{m}$ gebildet sind.

Es ist daher $x=y$. Nun ist die Concentrationsabnahme von $M_{1}$ gegeben durch $\xi_{1}=x-y=0$. Die Concentration von $M_{1}$ ist"also unveränderlich.

8.46. Ein ganz einfaches Beispiel ist ferner das bereits am Schlusse des $\$ .34$ erwähnte Reactionspaar $M_{1} \rightarrow M_{2}$ und $M_{2} \rightarrow M_{1}$. Bei diesem tritt ein constantes Geschwindigkeitsverhältnis auf, wenn die Anfangsconcentrationen dem Gleichgewichte entsprechen $\left(k_{1} A_{1}=k_{2} A_{2}\right)$. Gleichung 18) führt zul derselben Bedingung. Es ist also die Concentration von $M_{1}$ (und ebenso von $M_{2}$ ) bei Erfüllung der Gleichung $k_{1} A_{1}=k_{2} A_{2}$ unveränderlich, was in diesem Falle selbstverständlich ist.

§. 47. Die im vorstehenden entwickelten Bedingungen sind die einzigen, unter denen die Concentration der Molekelart $M_{1}$ unverändert bleibt, wenn ihre Concentration nur durch zwei Reactionen verändert wird. Dagegen muss ich es dahingestellt 
sein lassen, ob das Aultreten eines constanten Geschwindigkeitsverhältnisses $z$ wischen allen die Concentration von $M_{1}$ verändernden Reactionen nöthig ist, wenn ihre Zahl größer als zwei ist. Nur die Erfüllung der Gleichung 18) ist unter allen Umständen eine nothwendige Bedingung für das Auftreten einer unveränderlichen Concentration. Das ergibt sich aus folgendem.

Für das Auftreten einer unveränderlichen Concentration von $M_{1}$ ist die Erfüllung der Gleichung 17) für jedes $t$ die nothwendige und hinreichende Bedingung. Nothwendig (aber nicht hinreichend) ist daher die Erfüllung der Gleichung 17) für $t=0$. Setzt man in Gleichung 17) die Werte von $\frac{d x}{d t}$ u. s. w. für $t=0$ ein, welche man erhält, wenn man in den Gleichungen 3) die $\xi$ gleich Null setzt, so kommt man zur Gleichung 18), welche daher eine nothwendige Bedingung für das Auftreten einer unveränderlichen Concentration von $M_{1}$ ist. Neben Gleichung 18) muss aber noch eine Beziehung zwischen den Reactionsgeschwindigkeiten bestehen.

Sind nur. zwei Reactionen in Betracht zu ziehen, so folgt aus Gleichung 17), unmittelbar $\frac{d x}{d t}: \frac{d y}{d t}=-\frac{o_{1}-\beta_{1}}{n_{1}-\alpha_{1}}$., Die Reactionsgeschwindigkeiten müssen also in einem constanten Verhältnisse stehen.

Sind dagegen mehr als zwei Reactionen in Betracht zu ziehen, so können vielleicht auch andere Beziehungen zwischen den Reactionsgeschwindigkeiten existieren, welche zu unveränderlichen Concentrationen führen. Die Untersuchung der Natur dieser Beziehungen muss ich Mathematikern überlassen.

Jedenfalls kann es sich nur um singuläre Fälle handein. $\mathrm{Zu}$ ihrer Aufsuchung kann Gleichung 18) dienen, ähnlich wie Gleichung 13) zur Aufsuchung von Fällen mit constantem Geschwindigkeitsverhältnisse benützt werden kann.

\$. 48. Bei Erfüllung der Gleichung 18) (\$.41) wird ein Glied der Gleichung 13) (\$.30) gleich Null. Wird daher die Gleichung 13) zum Aufsuchen von Reactionspaaren mit constantem Geschwindigkeitsverhältnisse benützt, so ist eine gesonderte Untersuchung; $o b$ eine der betheiligten Molekelarten 
gemäß den Bedingungen des Abschnittes $V$ von unveränderlicher Concentration ist, nicht nöthig, obwohl Stoffe von constanter Concentration bei der Aufsuchung constanter Geschwindigkeitsverhältnisse außer Betracht zu bleiben haben (§.17). Denn die auf derartige Stoffe von constanter Concentration bezüglichen Glieder fallen aus Gleichung 13) von selbst heraus, beziehungsweise die Gleichung zerfällt in zwei oder mehr Gleichungen, die zum Theile die Bedingungen für constante Concentrationen von Molekelarten ausdrücken.

§. 49. In ähnlicher Weise, wie man ermitteln kann, unter welchen Umständen die Concentration einer Molekelart unveränderlich ist, kann man unter Berücksichtigung des $\S .5$ auch untersuchen, unter welchen Bedingungen die Concentration einer hylotropen Gruppe unveränderlich ist.

Eine nothwendige, aber nicht immer hinreichende Bedingung ist die Erfüllung einer der Gleichung 18) ähnlichen Gleichung, in welcher nur statt der Coefficienten $\left(n_{1}-\alpha_{1}\right)$ u. s. w. Coefficienten von der Form $\left(\frac{n_{1}-\alpha_{1}}{\nu_{1}}+\frac{n_{m+1}-\alpha_{m+1}}{\gamma_{m+1}}\right)$ auftreten. Die $v$ und $m$ haben dieselbe Bedutung in $\S .5$.

Ist die abgeänderte Gleichung 18) erfüllt und bestehen außerdem zwischen jenen Reactionen, welche die Concentration der hylotropen Gruppe ändern, constante Geschwindigkeitsverhältnisse, so ist die Concentration der hylotropen Gruppe unveränderlich. Bezüglich der Frage, ob auch bei anderen Beziehungen zwischen den in Betracht kommenden Reactionsgeschwindigkeiten constante Concentrationen möglich sind, gilt das in $\$ .47$ Gesagte.

Die zum Gleichgewichte zwischen den Molekelarten der hylotropen Gruppen führenden Reactionspaare ändern die Gesammtconcentration der hylotropen Gruppe nicht und gehören daher nicht $z u$ den Reactionen, welche bei Aufsuchung der Bedingungen für unveränderliche Concentrationen hylotroper Gruppen zu berücksichtigen sind.

Ein einfaches Beispiel bieten die Reactionen

$$
\begin{aligned}
\text { I. } & M_{1}+M_{4} \rightarrow M_{3}+M_{4} \\
\text { II. } & M_{1}+M_{4} \rightarrow M_{1}+M_{2}
\end{aligned}
$$




$$
\begin{array}{ll}
\text { III. } & M_{1} \rightarrow M_{2} \\
\text { IV. } & M_{2}^{*} \rightarrow M_{1},
\end{array}
$$

deren Veränderliche mit $x, y, z, t$ berechnet werden sollen.

$M_{1}$ und $M_{2}$ sind die Molekelarten der hylotropen Gruppe. Die Gesammtconcentration der Gruppe wird dargestellt durch die Summe der Concentrationen von $M_{1}$ und $M_{2}$.

Die Reactionen III und IV suchen zwischen diesen beiden Molekelarten ein Gleichgewicht herzustellen und ändern die Gesammtconcentration der hylotropen Gruppe nicht.

Dagegen ändern die Reactionen I und II die Gesammtconcentration der hylotropen Gruppe. Da nur zwei Reactionen in Betracht kommen, müssen diese in einem constanten Geschwindigkeitsverhältnisse stehen, wenn die Concentration der hylotropen Gruppe $\left(M_{1} M_{2}\right)$ unveränderlich sein soll. Das ist hier wegen Gleichheit der linken Seiten der vollständigen Reactionsgleichungen (\$. 20) der Fall.

Außerdem muss die abgeänderte Gleichung 18) erfüllt sein; auch in diese Gleichung gehen nur die Reactionen I und II ein. $\nu_{1}$ und $v_{m+1}$ sind gleich eins. Die Molekelart, welche in $\S .5$ den Index $m+1$ hatte, hat hier den Index 2. Man erhält also $0=k_{1} A_{1} A_{4}-k_{2} A_{1} A_{4}$ oder $k_{1}=k_{2}$.

Unter diesen Umständen ist $x=y$. Da nun $\xi_{1}=x+z-u$ und $\xi_{2}=u-y-z$ ist, ist $\xi_{1}+\xi_{2}=0$, daher die Gesammtconcentration der hylotropen Gruppe unveränderlich.

Befinden sich unter den Molekelarten Ionen, so ist $\mathrm{zu}$ berücksichtigen, dass nur jene Reactionsgleichungen möglich sind, welche nicht das Auftreten freier elektrischer Ladungen bedingen (§.1).

\section{Constante Umwandlungsverhältnisse.}

§. 50. Als "Umwandlungsverhältnis « hat Skraup ${ }^{1}$ in einem speciellen Falle das Verhältnis bezeichnet, in welchem ein Stoff durch zwei gleichzeitig verlaufende Reactionen verbraucht wird. Ich will im folgenden diesen Ausdruck in einer etwas allgemeineren Bedeutung gebrauchen.

1 Monatshefte für Chemie, 20, 587 (1899): 
Als Umwandlungsverhältnis bezeichne ich das Verhältnis der Concentrationsabnahmen zweier Stoffe, die im selben System durch Reactionen gebildet oder verbraucht werden, wobei Concentrationszunahmen als negative Concentrationsabnahmen $\mathrm{zu}$ behandeln sind. Die Umwandlungsverhältnisse werden also negativ sein, wenn einer der Stoffe gebildet, der andere verbraucht wird.

Die Frage, unter welchen Umständen Umwandlungsverhältnisse von der Zeit unabhängig sein können, ist wichtig geworden, seitdem Skraup in der erwähnten Arbeit das Auftreten eines constanten Umwandlungsverhältnisses bei der Cinchoninumlagerung dargethan hat. ${ }^{1}$ Ich habe diese Frage durch die Aufstellung des folgenden Satzes $\mathrm{zu}$ beantworten gesucht: ${ }^{2}$ "Ein von der Zeit unabhängiges Verhältnis zwischen den Mengen der durch zwei Reactionen erzeugten Producte beweist immer, dass Reactionen mit Nebenwirkungen vorliegen (da bei Gegenwirkungen und Folgewirkungen ein constantes Verhä1tnis unmöglich ist), und dass die Ausdrücke für die Geschwindigkeiten der beiden Reactionen sich nur durch einen von der Zeit unabhängigen Factor unterscheiden «.

Dieser Satz bedarf indes einer kleinen Einschränkung, wie ich bereits in der Abhandlung über die Umlagerung des Cinchonins erwähnt habe. Es war bisher kein Weg bekannt, um Fragen wie die nach den Bedingungen für das Auftreten eines constanten Umwandlungsverhältnisses systematisch zu untersuchen. Gerade darum empfand ich das Bedürfnis nach einem Schema, welches alle möglichen Reactionstypen als specielle Fälle in sich schließt. Nachdem ich das im Abschnitte I dieser Abhandlung gegebene Schema gefunden hatte, war es möglich, die Frage der constanten Umwandlungsverhältnisse systematisch zu behandeln. Im folgenden werden die Bedingungen für das Auftreten eines constanten Umwandlungsverhältnisses entwickelt. Die Erörterungen der Abschnitte IV

1 Verg1. meine Abhandlung: :Uber die Umlagerung des Cinchonins (ein Beitrag zur Theorie der katalytischen Wirkung) . Monatshefte für Chemie, 21 , 385 (1900); Zeitschrift für physikal. Chemie, 34, 290.

2 Zeitschrift für phyșikal. Chemie, 30, 599 (1899). 
und $V$ sind hauptsächlich als nothwendige Vorarbeiten für diesen Zweck durchgeführt worden.

§. 51. In den hier gewählten Bezeichnungen wird das Umwandlungsverhältnis zwischen den Molekelarten $M_{1}$ und $M_{2}$ ausgedrückt durch $\xi_{1}: \xi_{2}$, da die $\xi$ die Concentrationsabnahmen bedeuten (\$.4).

Ein von der Zeit unabhängiges Umwandlungsverhältnis wird daher dargestellt durch $\xi_{1}: \xi_{2}=C$, wo $C$ von der Zeit unabhängig ist. Daraus folgt

$$
\frac{d \xi_{1}}{d t}: \frac{d \xi_{2}}{d t}=C .
$$

Beide Gleichungen sind immer gleichzeitig erfüllt. Denn die bei der Integration der Gleichung 22) auftretende willkürliche Constante ist im vorliegenden Falle Null, da für $t=0 \xi_{1}$ und $\xi_{2}$ gleichzeitig Null werden. Die Erfüllung der Gleichung 22) ist daher die nothwendige und hinreichende Bedingung für das Auftreten eines von der Zeit unabhängigen Umwandlungsverhältnisses zwischen den Molekelarten $M_{1}$ und $M_{2}$.

8. 52. Führt man in die Gleichung 22) die Werte der Differentialquotienten ein, welche sich durch Differentiation der Gleichungen 2) (\$. 4) ergeben, so erhält man

$$
\begin{aligned}
& \frac{d \xi_{1}}{d t}: \frac{d \xi_{2}}{d t}= \\
& =\frac{\left(n_{1}-\alpha_{1}\right) \frac{d x}{d t}+\left(o_{1}-\beta_{1}\right) \frac{d y}{d t}+\left(p_{1}-\gamma_{1}\right) \frac{d z}{d t}+\ldots}{\left(n_{2}-\alpha_{2}\right) \frac{d x}{d t}+\left(o_{2}-\beta_{2}\right) \frac{d y}{d t}+\left(p_{2}-\gamma_{2}\right) \frac{d z}{d t}+\ldots}=C .
\end{aligned}
$$

Wenn diese Gleichung erfüllt, also das Umwandlungsverhältnis von der Zeit unabhängig ist, so erhält man den Wert von $C$, indem man $t=0$ setzt. Dann ist $x=y=\ldots=0$. Man erhält dann ebenso wie in $\S .30$

$$
C=\frac{\left(n_{1}-\alpha_{1}\right) k_{1} A_{1}^{n_{1}} A_{2}^{n_{2}} \ldots+\left(o_{1}-\beta_{1}\right) k_{2} A_{1}^{o_{1}} A_{2}^{o_{2}} \ldots+\ldots}{\left(n_{2}-\alpha_{2}\right) k_{1} A_{1}^{n_{1}} A_{2}^{n_{2}} \ldots+\left(o_{2}-\beta_{2}\right) k_{2} A_{1}^{o_{1}} A_{2}^{o_{2}} \ldots+\ldots}
$$


8. 53. Soll das constante Umwandlungsverhältnis nicht zwischen zwei Molekelarten, sondern zwischen hylotropen Gruppen bestehen, so bekommt man Bedingungsgleichungen von derselben Form; nur treten statt der $\left(n_{1}-\alpha_{1}\right)$ u. s. w. Coefficienten von der Form $\left(\frac{n_{1}-\alpha_{1}}{v_{1}}+\frac{n_{m+1}-\alpha_{m+1}}{v_{m+1}}\right)$ u. s. w. auf, wie aus den in $\$ .5$ gegebenen Erörterungen unmittelbar ersichtlich ist.

Die Reactionen mit Gegenwirkungen zwischen den Molekelarten der hylotropen Gruppen brauchen dabei nicht berücksichtigt zu werden, wie bereits in $\$ .5$ nachgewiesen wurde.

Im folgenden wird die Untersuchung hauptsächlich für constante Umwandlungsverhältnisse zwischen Molekelarten durchgeführt. Die Betrachtungen gelten aber auch für hylotrope Formen, soweit es sich nicht um die in Gleichung 23) auftretenden Coefficienten $\left(n_{1}-\alpha_{1}\right)$ u. s. w. handelt. Die entsprechenden Änderungen sind leicht durchzuführen.

§. 54. Der Vollständigkeit halber sei zuerst erwähnt, dass sich ein constantes Umwandlungsverhältnis Null oder $\infty$ ergibt, wenn eines der $\frac{d \xi}{d t}$ Null oder $\infty$ wird.

Nullwerden eines $\frac{d \xi}{d t}$ bedeutet, dass eine der Molekelarten, auf welche sich das Umwandlungsverhältnis bezieht, keine Concentrationsveränderung erleidet. Das tritt ein, wenn diese Molekelart an den stattfindenden Reactionen nicht oder lediglich als Katalysator betheiligt ist (z. B. $n_{1}=\alpha_{1}, o_{1}=\beta_{1}$ u. s. w.) oder wenn jene Réactionen, welche einzeln ihre Concentration ändern, gemäß Abschnitt $\mathrm{V}$ zusammen die Concentrationsänderung Null geben.

(Bei hylotropen Gruppen tritt das Nullwerden der gesammten Concentrationsänderung entweder nach Abschnitt $V$ ein oder durch Erfüllung von Bedingungen der Form $\frac{n_{1}-\alpha_{1}}{y_{1}}+$ $+\frac{n_{m+1}-\alpha_{m+1}}{y_{m+1}}=0$. Letztere Bedingung ist für eine Reaction nicht bloß dann erfüllt, wenn die Molekelarten $M_{1}$ und $M_{m+1}$ lediglich als Katalysatoren wirken, sondern auch bei Reactionsgleichungen von der Form 


$$
\begin{aligned}
n_{1} M_{1}+n_{n+1} M_{m+1}+X \rightarrow & \alpha_{1} M_{1}+ \\
& +\left(\frac{y_{m+1}\left(n_{1}-\alpha_{1}\right)}{\nu_{1}}+n_{m+1}\right) M_{n+1}+Y .
\end{aligned}
$$

Beispiel: $M_{1}+X \rightarrow M_{m+1}+Y$, wenn $\nu_{1}=\nu_{m+1}$. Es ist unmittelbar ersichtlich; dass diese Reaction die Concentration der hylotropen Gruppe nicht ändert.)

Ein $\frac{d \xi}{d t}$ kann unendlich werden, wenn Reactionen vorhanden sind, welche die Concentration des betreffenden Stoffes vermindern und mit sehr großer Geschwindigkeit verlaufen. Dann tritt der Stoff überhaupt nicht auf, da er sofort weiter umgewandeit wird.

Verlaufen mehrere Reactionen mit sehr großer Geschwindigkeit, welche theils die Molekelart erzeugen, theils verbrauchen (wie bei elektrolytischen Dissociationen), so braucht $\frac{d \tilde{\xi}}{d t}$ selbstverständlich nicht $\infty$ zu werden.

Die Umwandlungsverhältnisse $\frac{0}{0}$ und $\frac{\infty}{\infty}$ sind ohne Interesse.

§.55. Ein endliches, von der Zeit unabhängiges Verhältnis ergibt sich, wenn aus Gleichung 23) alle Functionen der Zeit herausfallen. Die in der Gleichung vorkommenden Zeitfunctionen sind die Geschwindigkeiten der nebeneinander verlaufenden Reactionen.

\$. 56. Reactionen, welche die Concentrationen der Stoffe $M_{1}$ und $M_{2}$ nicht ändern, haben auf das Umwandlungsverhältnis dieser Stoffe keinen Einfluss.

Damit eine einzelne Reaction aus der Gleichung 23) herausfällt, ist nothwendig, dass ihre Geschwindigkeit im Zähler und Nenner mit Null multipliciert ist. $\frac{d x}{d t}$ fällt heraus, wenn $n_{1}-\alpha_{1}=$ $=n_{2}-\alpha_{2}=0$. Dann sind sowohl $M_{1}$, als $M_{2}$ bei dieser Reaction nur Katalysatoren. (Bezüglich der Abänderung dieser zwei Bedingungen bei hylotropen Gruppen-gilt dasselbe wie in §. 54.)

Es können aber auch Gruppen von Reactionen aus Gleichung 23) herausfallen, wenn sie die Concentrationen beider Stoffe $\left(M_{1}\right.$ und $\left.M_{2}\right)$ gemäß Abschnitt $V$ unverändert 
lassen. Es muss also zwischen diesen Reactionen ein constantes Geschwindigkeitsverhältnis bestehen und außerdem müssen Zw ei Gleichungen von der Form der Gleichung 18) erfüllt sein.

Ein einfaches Beispiel sind die Reactionen

$$
M_{1}+M_{3} \rightarrow M_{2}+M_{4} \text { und } M_{2}+M_{3} \rightarrow M_{1}+M_{5}
$$

für $k_{1} A_{1}=k_{2} A_{2}$. Unter dieser Bedingung lassen die beiden Reactionen die Concentrationen von $M_{1}$ und $M_{2}$ unverändert. Denn Gleichung 18) ergibt sowohl für die Unveränderlichkeit der Concentration von $M_{1}$, als auch für die von $M_{2}$ diese Bedingung; die Constanz des Geschwindigkeitsverhältnisses der beiden Reactionen tritt aber bei unveränderlicher Concentration von $M_{1}$ und $M_{2}$ nach $\S .20$ ein, da der einzige, auf den linken Seiten der Reactionsgleichungen auftretende Stoff von veränderlicher Concentration $\left(M_{3}\right)$ in beiden Gleichungen denselben Coefficienten hat. Durch Integration der Geschwindigkeitsgleichungen überzeugt man sich leicht, dass in diesem Falle $x=y$ und demgemä $\beta$ die Concentrationen von $M_{1}$ und $M_{2}$ unveränderlich sind. Diese beiden Reactionen kommen also bei $k_{1} A_{1}=k_{2} A_{2}$ nicht in Betracht, wenn noch andere Reactionen die Concentrationen von $M_{1}$ und $M_{2}$ verändern und die Constanz des Umwandlungsverhältnisses für diese Molekelarten untersucht werden soll.

Man sieht, dass Reactionen, welche aus Gleichung 23) herausfallen, auch in Gleichung 24) verschwinden, also den Zahlenwert eines constanten Umwandlungsverhältnisses nicht beeinflussen.

§. 57. Wenn alle stattfindenden Reactionen unter $\$ .56$ fallen, so ist die Concentration von $M_{1}$ und $M_{2}$ unveränderlich und das Umwandlungsverhältnis ist $\frac{0}{0}$. Von Interesse sind nur bestimmte und endliche Umwandlungsverhältnisse. Solche können nur auftreten, wenn nach Weglassung der dem \$. 56 entsprechenden Reactionen noch andere übrig bleiben. Die Zahl der übrig bleibenden Reactionen kann eins oder größer sein.

§. 58. Bleibt nur eine Reaction übrig, so gehen Gleichung 23) und 24) über in $\frac{d \xi_{1}}{d t}: \frac{d \xi_{2}}{d t}=\frac{n_{1}-\alpha_{1}}{n_{2}-\alpha_{2}}=C$. Das Umwandlungs- 
verhältnis ist dann unter allen Umständen constant. (Das gilt auch für hylotrope Gruppen; nur ist die Form von $C$ nach $\S .53$ eine andere.) Dieses Ergebnis ist selbstverständlich. Denn wenn die Mengen zweier Stoffe durch eine einzige Reaction verändert werden, stehen die umgewandelten Mengen in einem stöchiometrischen, durch die Reactionsgleichung angegebenen Verhältnisse.

§. 59. Bleibt nach Weglassung der unter $\$ .56$ fallenden Reactionen mehr als eine Reaction übrig, so kann Gleichung 23) auf zwei Arten befriedigt werden: 1. wenn jedes Glied im Zähler und Nenner durch Division mit $\frac{d x}{d t}$ constant wird; 2. wenn Zähler und Nenner die Form besitzen

$$
\left(\frac{d x}{d t}+\mu_{2} \frac{d y}{d t}+\cdots\right) \times \text { Const. }
$$

wo $\mu_{2}$ u. s. w. im Zähler und Nenner dieselben Werte haben.

Sind mehr als zwei Reactionen zu berücksichtigen, so sind auch Lösungen denkbar, welche unter keine der beiden Arten fallen.

§. 60. Die im vorigen Paragraphen erwähnte erste Art, die Gleichung 23) zu befriedigen, führt auf

$$
\frac{d x}{d t}: \frac{d y}{d t}=K_{1}, \frac{d x}{d t}: \frac{d z}{d t}=K_{2} \text { u. s.w., }
$$

wo $K_{1}, K_{2} \ldots$ Constante sind. Unter welchen Umständen diese Bedingungen erfüllt sind, ist in Abschnitt IV besprochen worden.

Man kommt also zu folgendem Satze: Ein constantes Umwandlungsverhältnis zwischen zwei Stoffen tritt auf, wenn Zwischen sämmtlichen Reactionen, welche nicht einzeln oder gruppenweise die Concentration beider Stoffe unverändert lassen, constante Geschwindigkeitsverhältnisse bestehen.

Wenn daher die Geschwindigkeitsverhältnisse zweier oder mehrerer neben einander verlaufender Reactionen von der Zeit unabhängig sind, so sind auch die Umwandlungsverhältnisse aller jener Stoffe von der Zeit unabhängig, deren Concentrationsänderungen lediglich von den Reactionen mit constanten Geschwindigkeitsverhältnissen abhängen. 
Die in den $\S .20$ bis 37 gegebenen Beispiele von Reactionspaaren mit constantem Geschwindigkeitsverhältnisse sind daher sämmtlich zugleich auch Beispiele für constante Umwandlungsverhältnisse $z$ wischen allen in ihnen vorkommenden Molekelarten, wenn daneben keine anderen auf diese Molekelarten bezüglichen Reactionen stattfinden.

Bei jenen Reactionspaaren, welche ein constantes Geschwindigkeitsverhältnis infolge Gleichheit der linken Seiten der vollständigen Reactionsgleichungen haben ( $\$ .20$ bis 26), wird das constante Umwandlungsverhältnis zwischen zwei Molekelarten in keiner Weise gestört durch andere daneben verlaufende Reactionen, wenn letztere in ihrer Gesammtheit auf die Concentrationsänderungen der beiden Molekelarten ohne Einfluss sind ( $\$ .56)$, da das constante Geschwindigkeitsverhältnis hiedurch nicht berührt wird.

Beispiel: Finden nur die Reactionen $M_{1}+M_{2} \rightarrow M_{3}$ und $M_{1}+M_{2} \rightarrow M_{4}$ statt, so treten zwischen allen vier Stoffen constante Umwandlungsverhältnisse auf. Das Hinzutreten der Reaction $M_{2} \rightarrow M_{5}$ stört zwar die constanten Umwandlungsverhältnisse, an denen $M_{2}$ betheiligt ist, aber nicht jene zwischen $M_{1}, M_{3}$ und $M_{4}$.

Wenn dagegen die Constanz des Geschwindigkeitsverbältnisses gemäß $\S .37$ eintritt, sind weitere daneben verlaufende Reactionen nur dann ohne Einfluss, wenn sie auf die Concentrationsänderungen sämmtlicher auf den linken Seiten der vollständigen Reactionsgleichungen des betrachteten Reactionspaares vorkommen den Molekelarten ohne Einfluss sind; anderenfalls. ändern sich die Bedingungen für das constante Geschwindigkeitsverhältnis (\$.38) und demgemäß auch die Bedingungen für das constante Umwandlungsverhältnis.

§.61. Der Wert des constanten Umwandlungsverhältnisses ist durch Gleichung 24) (\$.52) gegeben. Man sieht, dass er im allgemeinen von den Anfangsconcentrationen abhängt.

Das Umwandlungsverhältnis ist jedoch von den Anfangsconcentrationen unabhängig, wenn es dadurch constant wird, dass die Geschwindigkeitsverhältnisse zwischen jenen Reactionen, welche weder die Concentrationen der beiden Molekelarten (einzeln oder gruppenweise) unverändert lassen 
und daher das Umwandlungsverhältnis überhaupt nicht beeinflussen ( $\$ .56$ ), noch die Anfangsgeschwindigkeit Null haben und daher in der Gleichung 24) überhaupt nicht auftreten, infolge Gleichheit der linken Seiten der vollständigen Reactionsgleichungen ( $\$ .20)$ constant werden.

Dann ist $n_{1}=o_{1}=p_{1}=\ldots$ und $n_{2}=o_{2}=p_{2}=\ldots$ Zähler und Nenner lassen sich durch $A_{1}^{n_{1}} A_{2_{2}}^{n_{2}} \ldots$ dividieren und man erhält

$$
C=\frac{\left(n_{1}-\alpha_{1}\right) k_{1}+\left(n_{1}-\beta_{1}\right) k_{2}+\ldots}{\left(n_{2}-\alpha_{2}\right) k_{1}+\left(n_{2}-\beta_{2}\right) k_{2}+\ldots}
$$

(Handelt es sich um hylotrope Gruppen, so treten wieder die in $\S .53$ erwähnten Coefficienten auf.)

Besteht Gleichheit der linken Seiten der vollständigen Reactionsgleichungen nur hinsichtlich der. Stoffe von veränderlicher Concentration, nicht aber hinsichtlich daneben vorhandener Molekelarten von constanter Concentration, so tritt ebenfalls ein constantes Umwandlungsverhältnis auf, welches jedoch von den constanten Concentrationen abhängt.

Ist für eine einzelne Molekelart die Bedingung $n_{1}=o_{1}=$ $=p_{1}=\ldots$ erfüllt, so fällt ihre Anfangsconcentration aus Gleichung 24) heraus.

Hat man daher ein constantes Umwandlungsverhältnis beobachtet, welches nicht stöchiometrischer Art ist $(\S .58,62)$, so kann man durch Abänderung der Anfangsconcentrationen einzelner Molekelarten ermitteln, ob diese Molekelarten auf den linken Seiten jener vollständigen Reactionsgleichungen, welche weder die Anfangsgeschwindigkeit Null haben, noch die Concentrationen der Molekelarten mit constantem Umwandlungsverhältnisse unverändert lassen, mit gleichen Coefficienten vorkommen oder nicht. Durch derartige Ermittlungen kann die Verwertung eines constanten Umwandlungsverhältnisses für die Feststellung der Natur der stattfindenden Reactionen sehr erleichtert werden. Ein von sämmtlichen Anfangsconcentrationen und der Zeit unabhängiges Umwandlungsverhältnis zwischen $z w e i$ Molekelarten, welches nicht ein stöchiometrisches 
ist, beweist die Gleichheit der linken Seiten der vollständigen Reactionsgleichungen all er Reactionen, welche die Mengen der zwei Molekelarten verändern und nicht die Anfangsgeschwindigkeit Null haben. Als Reactionen, welche die Concentrationen nicht ändern, sind auch solche zu betrachten, bei denen die entsprechenden Concentrationsänderungen sich gruppenweise aufheben. Die Anfangsgeschwindigkeit Null haben Reactionen, zu deren Eintritt die Gegenwart von Stoffen erforderlich ist, welche am Anfange nicht da sind, sondern erst während der Reaction gebildet werden. (Solche Reactionen können nicht nach $\S .60$, wohl aber nach $\S .68$ mit dem constanten Umwandlungsverhältnisse verträglich sein.)

Eine Einschränkung erleidet der besprochene Schluss von dem von den Anfangsconcentrationen unabhängigen Umwandlungsverhältnisse auf die Form der Reactionsgleichungen nur für Molekelarten, die mit anderen in einem sehr rasch sich einstellenden Gleichgewichte stehen. Nach $\$ .6 a$ und $26 a$ kann in diesem Falle die eine hylotrope Gruppe durch die andere ersetzt werden, ohne dass das constante Umwandlungsverhältnis gestört wird.

§. 62. Es soll nunmehr die zweite im $\$ .59$ erwähnte Möglichkeit für das Auftreten eines constanten Umwandlungsverhältnisses erörtert werden.

Enthalten Zähler und Nenner des Bruches in Gleichung 23) den Factor $\left(\frac{d x}{d t}+\mu_{2} \frac{d y}{d t}+\mu_{3} \frac{d z}{d t}+\ldots\right)$ multipliciert mit constanten Größen, so hat man

$$
\begin{array}{r}
\frac{d \xi_{1}}{d t}: \frac{d \xi_{2}}{d t}=\frac{K_{1} \frac{d x}{d t}+K_{1} \mu_{2} \frac{d y}{d t}+K_{1} \mu_{3} \frac{d z}{d t}+\ldots}{K_{2} \frac{d x}{d t}+K_{2} \mu_{2} \frac{d y}{d t}+K_{2} \mu_{3} \frac{d z}{d t}+\ldots}= \\
=\frac{K_{1}}{K_{2}}=C .
\end{array}
$$

Da die Gleichung 23) allgemein giltig ist, ist Gleichung 26) nur möglich, wenn folgende Gleichungen gelten 


$$
\begin{aligned}
& K_{1}=n_{1}-\alpha_{1}=\frac{o_{1}-\beta_{1}}{\mu_{2}}=\frac{p_{1}-\gamma_{1}}{\mu_{3}}=\ldots \\
& K_{2}=n_{2}-\alpha_{2}=\frac{o_{2}-\beta_{2}}{\mu_{2}}=\frac{p_{2}-\gamma_{2}}{\mu_{3}}=\ldots
\end{aligned}
$$

Daraus folgt

$$
C=\frac{K_{1}}{K_{2}}=\frac{n_{1}-\alpha_{1}}{n_{2}-\alpha_{2}}=\frac{o_{1}-\beta_{1}}{o_{2}-\beta_{2}}=\frac{p_{1}-\gamma_{1}}{p_{2}-\gamma_{2}}=\ldots
$$

Nun bedeutet $n_{1}-\alpha_{1}$ die Anzahl der nach der Reactionsgleichung I verschwindenden Molekeln von $M_{1}$, wobei gebildete Molekeln als negativ verschwindende zu betrachten sind. Die gleiche Bedeutung hat $o_{1}-\beta_{1}$ hinsichtlich des Stoffes $M_{1}$ und der Reaction II, $n_{2}-\alpha_{2}$ hinsichtlich des Stoffes $M_{2}$ und der Reaction I u. s. W.

Die Gleichung 27) sagt also: Ein constantes Umwandlungsverhältnis zwischen den Molekelarten $M_{1}$ und $M_{2}$ tritt auf, wenn beide Molekelarten durch sämmtliche Reactionen, welche ihre Concentrationen nicht (einzeln oder gruppenweise) unverändert lassen, in demselben stöchiometrischen Verhältnisse aufgebraucht oder gebildet werden.

(Hinsichtlich hylotroper Gruppen ist wieder auf $\S .53 \mathrm{zu}$ verweisen.)

§. 63. Da Bildung als negativer Aufbrauch zu betrachten ist, tritt das constante Umwandlungsverhältnis auch dann auf, wenn die beiden Molekelarten bei einer Reaction gebildet, bei einer anderen in demselben stöchiometrischen Verhältnisse verbraucht werden, oder wenn bei einer Reaction der Stoff $M_{1}$ in den Stoff $M_{2}$, bei einer anderen umgekehrt $M_{2}$ in $M_{1}$ in demselben stöchiometrischen Verhältnisse umgewandelt wird.

\$. 64. Da in Gleichung 26) die Reactionsgeschwindigkeiten völlig willkürlich bleiben und außerdem nur die auf die Molekelarten $M_{1}$ und $M_{2}$ bezüglichen Coefficienten der Reactionsgleichungen auftreten, können neben den Molekelarten $M_{1}$ und $M_{2}$ beliebige andere Molekelarten mit beliebigen Coefficienten in den Reactionsgleichungen auftreten, ohne dass das constante Umwandlungsverhältnis gestört wird. 
§. 65. Ein Beispiel für constantes Umwandlungsverhältnis nach $\$ .62$ bietet das Reactionspaar

$$
n_{1} M_{1}+n_{2} M_{2}+n_{3} M_{3}+\ldots \rightarrow \alpha_{1} M_{1}+\alpha_{2} M_{2}+\alpha_{3} M_{3}+\ldots
$$

und

$$
\begin{aligned}
a\left(n_{1}+b\right) M_{1}+a\left(n_{2}+c\right) M_{2}+o_{3} M_{3}+ & \ldots \rightarrow a\left(\alpha_{1}+b\right) M_{1}+ \\
& +a\left(\alpha_{2}+c\right) M_{2}+\beta_{3} M_{3}+\ldots
\end{aligned}
$$

Das Umwandlungsverhältnis ist für die Molekelarten $M_{1}$ und $M_{2}$ und nur für diese von der Zeit unabhängig, wenn nicht etwa auch die $n_{3}, n_{4} \ldots, o_{3}, o_{4} \ldots, \alpha_{3} \ldots, \beta_{3} \ldots$ der Bedingung 27) genügen. Gemäß $\S .64$ hindern beliebige Werte von. $n_{3}, o_{3}, \alpha_{3}, \beta_{3}$ u. s. w. die Constanz des Umwandlungsverhältnisses für $M_{1}$ und $M_{2}$ nicht.

Der erste im $\$ .63$ erwähnte Fall liegt beispielsweise für die Molekelarten $M_{1}$ und $M_{2}$ vor bei den Reactionsgleichungen

$$
M_{3} \rightarrow M_{1}+2 M_{2} \text { und } 2 M_{1}+4 M_{2} \rightarrow M_{5} .
$$

Der zweite Fall des $\S .63$ liegt bei allen Gegenwirkungen vor, und zwar hinsichtlich aller betheiligten Molekelarten. Das allgemeine Schema für Gegenwirkungen ist

$$
v_{1} M_{1}+\ldots+v_{m} M_{m} \rightleftarrows v_{m+1} M_{m+1}+\ldots+v_{m+-n} M_{m+n} .
$$

Für jede Molekelart wird $n_{a}=\beta_{a}, \alpha_{a}=o_{a}$, wobei außerdem eines der beiden Coefficientenpaare Null ist. Gleichung 27) geht daher über in die Identität

$$
\frac{n_{a}-\alpha_{a}}{n_{b}-\alpha_{b}}=\frac{\alpha_{a}-n_{a}}{\alpha_{b}-n_{b}}
$$

Durch das Hinzutreten weiterer Reactionen von anderer Beschaffenheit hört die Constanz des Umwandlungsverhältnisses bei allen angeführten Beispielen auf.

§. 66. Das constante Umwandlungsverhältnis nach $\S .62$ stellt eine rein stöchiometrische Beziehung dar, wie aus Gleichung 27) unmittelbar ersichtlich ist, und gleicht daher dem im $\$ 58$ besprochenen Falle. 
§.67. Haben nur zwei Reactionen auf das Umwandlungsverhältnis Einfluss, so sind die in den $\S \S .60$ bis 61 und 62 bis 66 besprochenen Fälle die einzigen, in denen das Verhältnis von der Zeit unabhängig wird.

Bei zwei Reactionen folgt nämlich aus Gleichung 23)

$$
\frac{d x}{d t}: \frac{d y}{d t}=-\frac{\left(o_{1}-\beta_{1}\right)-C\left(o_{2}-\beta_{2}\right)}{\left(n_{1}-\alpha_{1}\right)-C\left(n_{2}-\alpha_{2}\right)} .
$$

Soll nun $C$ constant sein, so muss auch das Geschwindigkeitsverhältnis constant sein (Fall des $\S .60$ ), außer wenn die rechte Seite der Gleichung den unbestimmten Wert $\frac{0}{0}$ annimmt. Dann ist aber $C=\frac{o_{1}-\beta_{1}}{o_{2}-\beta_{2}}=\frac{n_{1}-\alpha_{1}}{n_{2}-\alpha_{2}}$, entsprechend $\S .62$.

Dasselbe gilt auch für Umwandlungsverhältnisse hylotroper Gruppen.

§. 68. Haben drei oder mehr Reactionen auf das Umwandlungsverhältnis Einfluss, so ist die Möglichkeit von constanten Umwandlungsverhältnissen, welche weder dem \$. 60, noch dem §. 62 entsprechen, nicht ausgeschlossen. Einen näheren Einblick bekommt man in folgender Weise.

Aus Gleichung 22) folgt durch Differentiation als nothwendige und hinreichende Bedingung für ein constantes endliches Umwandlungsverhältnis

$$
\left(\frac{d^{2} \xi_{1}}{d t^{2}}: \frac{d \xi_{1}}{d t}\right)-\left(\frac{d^{2} \xi_{2}}{d t^{2}}: \frac{d \xi_{2}}{d t}\right)=0
$$

oder

$$
\frac{d \xi_{2}}{d t} \cdot \frac{d^{2} \xi_{1}}{d t^{2}}-\frac{d \xi_{1}}{d t} \cdot \frac{d^{2} \xi_{2}}{d t^{2}}=0
$$

Führt man in diese Gleichung die aus den Gleichungen 2) folgenden Werte der Differentialquotienten ein, so erhält man nach gehörigem Ordnen, wobei die Glieder mit $\frac{d x}{d t} \cdot \frac{d^{2} x}{d t^{2}}$ herausfallen: 


$$
\begin{aligned}
& {\left[\left(n_{1}-\alpha_{1}\right)\left(o_{2}-\beta_{2}\right)-\left(n_{2}-\alpha_{2}\right)\left(o_{1}-\beta_{1}\right)\right]\left[\frac{d y}{d t} \cdot \frac{d^{2} x}{d t^{2}}-\frac{d x}{d t} \cdot \frac{d^{2} y}{d t^{2}}\right] } \\
+ & {\left[\left(n_{1}-\alpha_{1}\right)\left(p_{2}-\gamma_{2}\right)-\left(n_{2}-\alpha_{2}\right)\left(p_{1}-\gamma_{1}\right)\right]\left[\frac{d z}{d t} \cdot \frac{d^{2} x}{d t^{2}}-\frac{d x}{d t} \cdot \frac{d^{2} z}{d t^{2}}\right] } \\
+ & \ldots \ldots \ldots \ldots \ldots \ldots \ldots \ldots \ldots \ldots \ldots \ldots \ldots \ldots \ldots \ldots \ldots \ldots \ldots \ldots \ldots \ldots \ldots \ldots \ldots \ldots \ldots \ldots \ldots \ldots
\end{aligned}
$$

Die linke Seite dieser Gleichung enthält so viele Summanden, als man Reactionspaare bilden kann, also bei $n$ Reactionen $\frac{n(n-1)}{2}$ Glieder. Auch diese Gleichung ist eine nothwendige und hinreichende Bedingung für das Auftreten eines constanten Umwandlungsverhältnisses zwischen den Molekelarten $M_{1}$ und $M_{2}$.

§. 69. Gleichung 29) kann erstens dadurch befriedigt werden, dass jeder einzelne Summand Null wird. Letzteres kann geschehen, indem entweder alle Factoren von der Form $\left[\left(n_{1}-\alpha_{1}\right)\left(o_{2}-\beta_{2}\right)-\left(n_{2}-\alpha_{2}\right)\left(o_{1}-\beta_{1}\right)\right]$ oder alle Factoren von der Form $\left[\frac{d y}{d t} \cdot \frac{d^{2} x}{d t^{2}}-\frac{d x}{d t} \cdot \frac{d^{2} y}{d t^{2}}\right]$ Null werden. Der erste Fall entspricht dem $\S .62$, der zweite dem $\$ .60$, da aus

$$
\frac{d y}{d t} \cdot \frac{d^{2} x}{d t^{2}}=\frac{d x}{d t} \cdot \frac{d^{2} y}{d t^{2}}
$$

folgt

$$
\frac{d x}{d t}: \frac{d y}{d t}=\text { Const. }
$$

Das Nullwerden der einzelnen Summanden erfordert die Erfüllung von $\frac{n(n-1)}{2}$ Gleichungen, die aber nicht voneinander unabhängig sind, sondern aus den $n-1$ Gleichungen folgen, die sich nach $\S .60$ oder 62 ergeben.

§. 70. Es ist nicht möglich, die Gleichung 29) in der Weise zu befriedigen, dass ein Theil der Summanden ausschließlich nach $\$ .60$, der Rest ausschließlich nach $\S .62$ Null wird; vielmehr muss entweder $\$ .60$ oder $\S .62$ für alle Reactionen zutreffen. 
Um das einzusehen, denke man sich aus den stattfindenden Reactionen vier herausgegriffen, etwa die Reactionen I, II, III und IV. Für das Reactionspaar I, II sei nur die Bedingung des $\S$. 60, für III, IV nur die Bedingung des $\$ .62$ erfüllt. Eine der beiden Bedingungen muss aber auch für die Reactionspaare I, III, I, IV, II, III und II, IV erfüllt sein. Nimmt man an, dass für I, III der Fall des $\S .60$ eintrete, so folgt dasselbe für II. III. Dann muss noch für I, IV und II, IV eine Annahme gemacht werden. Nimmt man auch für I, IV den Fall des $\S .60$ an, so folgt das Gleiche für $\mathrm{II}_{2} \mathrm{IV}$. Es entsprechen also dann alle Reactionspaare dem Falle des $\S .60$. Nimmt man für I, IV den Fall des $\S .62$ an, so trifft das auch für I, III zu. Außerdem muss noch für II, IV eine Annahme gemacht werden. Je nachdem man die eine oder die andere der beiden möglichen Annahmen macht, entsprecher dann alle sechs Reactionspaare entweder dem Falle des $\$ .60$ oder 62.

Da die vier Reactionen beliebig gewählt werden können und mit den übrigen Reactionen ebenfalls durch Bedingungen verknüpft sein müssen, gilt also allgemein, dass alle Reactionen entweder dem $\S .60$ oder dem $\S .62$ entsprechen müssen. Daneben können einige Reactionen außerdem noch dem zweiten Falle entsprechen.

§. 71. Die Gleichung 29) kann möglicherweise auch befriedigt werden, ohne dass alle einzelnen Summanden Null werden. Da die Gleichung für jedes $t$ gelten muss, muss sie auch für $t=0$ gelten. Man bekommt daher durch Einsetzen der betreffenden Werte eine allgemein giltige und nothwendige, aber nicht hinreichende Bedingung für das Auftreten eines constanten Umwandlungsverhältnisses.

$Z \mathrm{u}$ diesem $Z_{w}$ ecke sind zunächst die Werte von $\frac{d^{2} x}{d t^{2}}$ u. s.w. zu bilden. Man erhält durch Differentiation der Gleichungen 3)

$$
\frac{d^{2} x}{d t^{2}}=-\frac{d x}{d t}\left[\frac{n_{1}}{A_{1}-\xi_{1}} \cdot \frac{d \xi_{1}}{d t}+\frac{n_{2}}{A_{2}-\xi_{2}} \cdot \frac{d \xi_{2}}{d t}+\cdots\right]
$$

und hieraus durch Einführung der Werte für $\frac{d \xi_{1}}{d t}$ u. s. w. 


$$
\begin{aligned}
\frac{d^{2} x}{d t^{2}}=-\frac{d x}{d t}\{ & \left\{\frac{n_{1}\left(n_{1}-\alpha_{1}\right)}{A_{1}-\xi_{1}}+\frac{n_{2}\left(n_{2}-\alpha_{2}\right)}{A_{2}-\xi_{2}}+\ldots\right] \frac{d x}{d t}+ \\
& \left.+\left[\frac{n_{1}\left(o_{1}-\beta_{1}\right)}{A_{1}-\xi_{1}}+\frac{n_{2}\left(o_{2}-\beta_{2}\right)}{A_{2}-\xi_{2}}+\ldots\right] \frac{d y}{d t}+\ldots\right\} .
\end{aligned}
$$

Für $t=0$ hat man

$$
\begin{aligned}
& \left(\frac{d^{2} x}{d t^{2}}\right)_{t=0}= \\
& =-k_{1} A_{1}^{n_{1}} A_{2}^{n_{2}} \ldots\left\{\left[\frac{n_{1}\left(n_{1}-\alpha_{1}\right)}{A_{1}}+\frac{n_{2}\left(n_{2}-x_{2}\right)}{A_{2}}+\ldots\right] k_{1} A_{1}^{n_{1}} A_{2}^{n_{2}} \ldots+\right. \\
& \left.\quad+\left[\frac{n_{1}\left(o_{1}-\beta_{1}\right)}{A_{1}}+\frac{n_{2}\left(o_{2}-\beta_{2}\right)}{A_{2}}+\ldots\right] k_{2} A_{1}^{o_{1}} A_{2}^{o_{2}} \ldots+\ldots\right\} .
\end{aligned}
$$

Analog ist

$$
\begin{aligned}
& \left(\frac{d^{2} y}{d t^{2}}\right)_{f=0}= \\
& =-k_{2} A_{1}^{o_{1}} A_{2}^{o_{2}} \ldots\left\{\left[\frac{o_{1}\left(n_{1}-\alpha_{1}\right)}{A_{1}}+\frac{o_{2}\left(n_{2}-\alpha_{2}\right)}{A_{2}}+\ldots\right] k_{1} A_{1}^{n_{1}} A_{2}^{n_{2}} \ldots+\right. \\
& \left.+\left[\frac{o_{1}\left(o_{1}-\beta_{1}\right)}{A_{1}}+\frac{o_{2}\left(o_{2}-\beta_{2}\right)}{A_{2}}+\ldots\right] k_{2} A_{1}^{o_{1}} A_{2}^{o_{2}} \ldots+\ldots\right\}
\end{aligned}
$$

u. S. W.

Durch Einsetzung der Werte erhält man

$$
\begin{aligned}
& {\left[\frac{d y}{d t} \cdot \frac{d^{2} x}{d t^{2}}-\frac{d x}{d t} \cdot \frac{d^{2} y}{d t^{2}}\right]_{t=0}=-k_{1} k_{2} A_{1}^{n_{1}+o_{1}} A_{2}^{n_{2}}+o_{2} \ldots} \\
& \quad \ldots\left\{\left[\frac{\left(n_{1}-o_{1}\right)\left(n_{1}-\alpha_{1}\right)}{A_{1}}+\frac{\left(n_{2}-o_{2}\right)\left(n_{2}-\alpha_{2}\right)}{A_{2}}+\ldots\right] k_{1} A_{1}^{n_{1}} A_{2}^{i t_{2}} \ldots+\right. \\
& \left.+\left[\frac{\left(n_{1}-o_{1}\right)\left(o_{1}-\beta_{1}\right)}{A_{1}}+\frac{\left(n_{2}-o_{2}\right)\left(o_{2}-\beta_{2}\right)}{A_{2}}+\ldots\right] k_{2} A_{1}^{o_{1}} A_{2}^{o_{2}} \ldots+\ldots\right\}
\end{aligned}
$$

Ähnliche Gleichungen gelten für

$$
\left[\frac{d z}{d t} \cdot \frac{d^{2} x}{d t^{2}}-\frac{d x}{d t} \cdot \frac{d^{2} z}{d t^{2}}\right]_{t=0} \text { u. s. w. }
$$


Der Ausdruck in der großen Klammer ist identisch mit dem Ausdrucke in Gleichung 13), wie es sein muss, weil es sich in beiden Fällen um die Bedingung für das Auftreten constanter Geschwindigkeitsverhältnisse handelt.

Durch Einsetzung dieser Werte in die Gleichung 29) erhält man eine allerdings etwas umfangreiche Gleichung, deren Erfüllung für das Auftreten constanter Umwandlungsverhältnisse nothwendig, aber nicht hinreichend ist. Diese Gleichung kann ebenso zur Ermittlung von Formen von Reactionsgleichungen und Beziehungen zwischen den Geschwindigkeitsconstanten und Anfarigsconcentrationen dienen, welche zil constanten Umwandlungsverhältnissen führen, wie Gleichung 13) dasselbe für constante Geschwindigkeitsverhältnisse und Gleichung 18) für constante Concentrationen leistet.

Die durch Einführung von Gleichung 30) in Gleichung 29) erhältliche Bedingung lehrt, dass constante Umwandlungsverhältnisse, welche nicht den Bedingungen der $\$ .60$ und 62 entsprechen, nur für bestimmte Beziehungen zwischen den Geschwindigkeitsconstanten und Anfangsconcentrationen möglich sind. Jene Fälle von constanten Umwandlungsverhältnissen, welche nicht unter $\$ .60$ oder 62 fallen, sind singuläre Fälle, welche in der Regel keine chemische Wichtigkeit haben.

Für Umwandlungsverbältnisse hylotroper Gruppen gilt dasselbe; nur ändern sich die Coefficienten der Gleichungen 29) und 30 ).

3. 72. Zusammenfassend kann man sagen: Ein bestimmtes und endliches, von der Zeit unabhängiges Umwandlungsverhältnis zwischen zwei Stoffen tritt auf:

1. wenn die Concentrationen der beiden Stoffe nur durch eine Reaction ( $\$ .58$ ) oder durch mehrere, aber im gleichen stöchiometrischen Verhältnisse geändert werden (§. 62); das Umwandlungsverhältnis ist in diesen Fällen eine rein stöchiometrische Beziehung;

2. wenn die Geschwindigkeiten sämmtlicher Reactionen, welche die Concentrationen der beiden Stoffe ändern, in einem von der Zeit unabhängigen Verhältnisse stehen (§.60).

Außerdem - sind constante Umwandlungsverhältnisse bei singulären. Werten der Geschwindigkeitsconstanten und der 
Anfangsconcentrationen, welche nicht unter 1 oder 2 fallen, nicht ausgeschlossen, wenn die Zahl der Reactionen, welche die Concentrationen der beiden Stoffe ändern, mindestens drei ist ( $\$ .67$ bis 71 ).

Als Reactionen, welche die Concentrationen der beiden Stoffe nicht ändern, sind nicht bloß jene Reactionen zu betrachten, an welchen diese Stoffe überhaupt nicht oder bloß als Katalysatoren betheiligt sind, sondern auch Reactionen, deren Wirkungen sich hinsichtlich der Concentrationen der beiden Stoffe aufheben (\$. 56).

§. 73. Aus dem Vorhergegangenen ergibt sich die Abänderung, welche an dem von mir aufgestellten und im $\S .50$ erwähnten Satze über den Zusammenhang zwischen constanten Verhältnissen zwischen den Mengen der durch $z$ wei Reactionen erzeugten Producte und Nebenwirkungen vorzunehmen ist. Derartige constante Verhältnisse treten auch bei Folgewirkungen oder bei Reactionen auf, die hinsichtlich der verschwindenden Stoffe völlig verschieden sind, wenn die in $\$ .25$ und 26 erwähnten eigenthümlichen katalytischen Beeinflussungen hinzukommen, oder bei singulären Werten der Geschwindigkeitsconstanten und Anfangsconcentrationen. In letzterem Falle sind auch die Geschwindigkeitsgleichungen der beiden Reactionen nicht lediglich hinsichtlich der Constanten verschieden.

Man sieht zugleich, dass diese Abänderungen keine erhebliche Wichtigkeit gewinnen dürften.

§. 74. Als Beispiel soll nunmehr die Umlagerung des Cinchonins besprochen werden. Skraup hat in der im $\$ .50$ citierten Abhandlung nachgewiesen, dass bei der Einwirkung von Halogenwasserstoffsäuren auf Cinchonin bei niedriger Temperatur die Mengen des gebildeten Halogenwasserstoffadditionsproductes und des daneben entstehenden $\alpha$ - $i$-Cinchonins in einem von der Zeit und der Anfangsconcentration der Säure unabțängigen Verhältnisse stehen, solange nicht die Anlagerung von Halogenwasserstoff an das $\alpha$ - $i$-Cinchonin in erheblichem Maße eintritt. Ich habe daraus in einer ebenfalls im $\$ .50$ citierten Abhandlung den Schluss gezogen, dass die stattfindenden Reactionen den Gleichungen $\mathrm{Ci}+\mathrm{H} X \rightarrow \mathrm{C}_{19} \mathrm{H}_{23} X_{2} \mathrm{O}$ und $\mathrm{Ci} \rightarrow \mathrm{Ci}^{\prime}$ (katalytisch beschleunigt durch Halogenwasser- 
stoff) entsprechen, wo $X$ ein Halogenatom, $\mathrm{Ci}$ die Cinchoninmolekel, $\mathrm{Ci}^{\prime}$ die Molekel des a-i-Cinchonins bedeuten. Auf Grund dieser Annahme habe ich eine atomistisch-kinetische Auffassung der katalytischen Wirkung der Halogenwasserstoffsäure gegeben, welche auch auf andere katalytische Wirkungen übertragbar ist.

Hiebei war die Annahme zugrunde gelegt worden, dass aus dem constanten Umwandlungsverhältnisse auf das Vorliegen von Nebenwirkungen geschlossen werden könne. Diese Annahme bedarf, wie gezeigt, einer Abänderung. Ich will daher im folgenden die Frage, welche Reactionsgleichungen und sonstige Bedingungen mit dem Auftreten eines constanten Umwandlungsverhältnisses verträglich sind, unter Berüicksichtigung der Ergebnisse dieses Abschnittes untersuchen.

§. 75. Ich nehme im folgenden an, dass die Basen nur in Form ihrer Salze mit zwei Molekeln Halogenwasserstoff, und zwar theils undissociiert, theils in Form ihrer zweiwertigen Ionen vorhanden sind.

Es sollen bedeuten:

$M_{1}$ Cinchonindichlorhydrat, undissociiert,

$H_{2}$ a.i-Cinchonindichlorhydrat, undissociiert,

$M_{3}$ Hydrochlorcinchonindichlorhydrat, undissociert,

$M_{4}$ zweiwertige Cinchoninionen,

$M_{5}$ zweiwertige $\alpha$-i-Cinchoninionen,

$M_{6}$ zweiwertige Hydrochlorcinchoninionen,

$M_{7}$ Chlorwasserstoff, undissociiert,

$M_{8}$ Wasserstoffionen,

$M_{9}$ Chlorionen.

Was für Chlorwasserstoff gilt, ist selbstverständlich ohne weiteres auf die anderen Halogene zu übertragen.

Hydrolyse kommt wegen des großen Überschusses von Halogenwasserstoff nicht in Betracht.

Zwischen diesen Molekelarten sollen zunächst beliebige Reactionen stattfinden, entsprechend den voliständigen Reactionsgteichungen

$$
\begin{aligned}
& n_{1} M_{1}+n_{2} M_{2}+\ldots+n_{9} M_{9} \rightarrow \alpha_{1} M_{1}+\ldots+\alpha_{9} M_{9} \\
& o_{1} M_{1}+o_{2} M_{2}+\ldots+o_{9} M_{9} \rightarrow \beta_{1} M_{1}+\ldots+\beta_{9} M_{9} \\
& \ldots \ldots \ldots \ldots \ldots \ldots \ldots \ldots+\ldots \ldots \ldots+\ldots \ldots
\end{aligned}
$$


\$.76. Es handelt sich hier um ein Umwandlungsverhältnis zwischen hylotropen Gruppen. Die Concentrationsänderung des Cinchonins ist gegeben durch die Summe der Concentrationsänderungen von $M_{1}$ und $M_{4}$, die des $\alpha$ - $i$-Cinchonins durch die von $M_{2}$ und $M_{5}$, die des Hydrochlorcinchonins durch $M_{3}$ und $M_{6}$. Die Coefficienten $\nu_{1}$ und $v_{m+1}$. des. $\$ .5$ sind eins, da die für die Concentrationen der hylotropen Gruppen charakteristischen Molekelarten in den zu den Dissociationsgleichgewichten gehörigen Reactionsgleichungen immer mit dem Coefficienten eins auftreten. Statt der für Molekelarten giltigen Coefficienten $\left(n_{1}-\alpha_{1}\right)$ u. s. w. treten daher im volliegenden Falle Coefficienten von der Form $\left(n_{1}-\alpha_{1}+n_{4}-\alpha_{4}\right)$ u. s. w. auf (\$. 53). Übrigens ist es wegen des Principes der Einfachheit der Reactionsgleichungen ganz unwahrscheinlich, dass für die Bildung des Hydrochlorcinchonins und $\alpha-i$-Cinchonins Reactionsgleichungen in Betracht kommen, welche etwa zweiwertige Cinchoninionen und undissociiertes Cinchonindichlorhydrat nebeneinander enthalten.

Bezeichnet man mit $\zeta_{1}, \zeta_{2}, \zeta_{3}$ die gesammten Concent:ationsabnahmen des Cinchonins, $\alpha-i$-Cinchonins und Hydrochlorcinchonins, so geht die Gleichung 23) für das Umwandlungsverhältnis von $\alpha-i$-Cinchonin und Hydrochlorcinchonin über in

$$
\begin{aligned}
\frac{d \zeta_{2}}{d t}: & \frac{d \zeta_{3}}{d t}= \\
= & \frac{\left(n_{2}-\alpha_{2}+n_{5}-\alpha_{5}\right) \frac{d x}{d t}+\left(o_{2}-\beta_{2}+o_{5}-\beta_{5}\right) \frac{d y}{d t}+\ldots}{\left(n_{3}-\alpha_{3}+n_{6}-\alpha_{6}\right) \frac{d x}{d t}+\left(o_{3}-\beta_{3}+o_{6}-\beta_{6}\right) \frac{d y}{d t}+\ldots}
\end{aligned}
$$

In diese Formel treten nur jene Reactionen ein, welche a-i-Cinchonin oder Hydrochlorcinchonin erzeugen oder verbrauchen; die zu den Dissociationsgleichgewichten führenden Reactionen kommen in der Formel nicht vor, da sie die Concentrationen der hylotropen Gruppen nicht ändern $(\$ .53,56)$.

ร. 77. Die Gründe, warum das constante Umwandlungśverhältnis nicht als ein stöchiometrisches betrachtet. werden 
kant, habe ich bereits in der früheren Abhandlung aüseinandergesetzt. Es fallen daher die in $\$ .58$ und 62 besprochenen Möglichkeiten fort. Nach $\S .62$ ist nämlich erforderlich, dass Hydrochlorcinchonin und $\alpha$-i-Cinchonin an jeder der in Gleichung 31) eintretenden Reactionen, und zwar im selben stöchiometrischen Verhältnisse betheiligt sind; dieses stöchiometrische Verhältnis wäre zugleich das Umwandlungsverhältnis.

Es bleiben daher nur die Fälle der \$. 60 und $68 \mathrm{zu}$ besprechen. Das Problem vereinfacht sich dadurch, dass die Unabhängigkeit des Umwandlungsverhältnisses voh̀ der Säureconcentration festgestellt ist. "Es miss daher die Säure in allen Geschwindigkeitsgleichungen, welche Änderungen der Concentration des $\alpha$ - $i$-Cinchonins und Hydrochlorcinchonins hervorbringen und nicht zur Zeit Null die Geschwindigkeit Null ergeben, denselben Factor bedingen, beziehungsweise auf den linken Seiten der vollständigen Reactionsgleichungen aller in Gleichung 31) eintretenden Reactionen, deren Anfangsgeschwindigkeit nicht Null ist, in derselben. Molekelzahl vorkommen; nur kann die Concentration der dissociierten Säure durch das Product der Ionenconcentrationen entsprechend der Bedingung für das elektrolytische Gleichgewicht ersetzt werden $(\$ .61)$.

§. 78. Nach §. 60 ist zum Eintritte eines constanten Umwandlungsverhältnisses die Constanz der Geschwindigkeitsverhältnisse für alle in Gleichung 31) eintretenden Reactionen hinreichend. Diese Bedingung ist nach $\S .20$ erfüllt, wenn die linken Seiten der betreffenden vollständigen Reactionsgleichungen gleich sind. Nun können aber $\alpha-i$-Cinchonin und Hydrochlorcinchonin nicht auf den linken Seiten aller Reactionsgleichungen vorkommen, weil andernfalls nur bei Anwesenheit dieser Stoffe Reaction eintreten könnte; in Wirklichkeit beginnt sie aber in Abwesenheit dieser Stoffe. Cinchonin und Chlorwasserstoff müssen dagegen auf den linken Seiten der Reactionsgleichungen vorkommen, "da sie zum Eintreten der Reaction nothwendig sind. Man kommt daher nach $\$ .20$ zu den Reactionsgleichungen $\mathrm{Ci}+\mathrm{HCl} \rightarrow \mathrm{C}_{19} \mathrm{H}_{23} \mathrm{ClN}_{2} \mathrm{O}$ und $\mathrm{Ci}+\mathrm{HCl} \rightarrow \mathrm{Ci}^{\prime}+\mathrm{HCl}$, welche ich bereits früher als den Thatsachen entsprechend bezeichnet habe. Daneben können noch andere Katalysatoren 
angenommen werden, aber nur in gleicher Weise für beide Reactionen.

§. 79. Es könnten aber auch constante Geschwindigkeitsverhältnisse nach §. 27 in Frage kommen. Da hiebei alle stattfindenden Reactionen $z u$ berücksichtigen sind, empfiehlt es sich, entsprechend $\S$. $38 e$ die aus den elektrolytischen Dissociationen folgenden Gleichgewichtsbedingungen in die Geschwindigkeitsgleichungen der langsam verlaufenden Reactionen einzuführen und die den umgeformten Geschwindigkeitsgleichungen entsprechenden fingierten Reactionsgleichungen der weiteren Erörterung zugrunde zu legen. Hiebei soll für die elektrolytischen Dissociationen das Massenwirkungsgesetz (beziehungsweise das Ostwald'sche Verdünnungsgesetz) angewendet werden. ${ }^{1}$

Nach $\S .26 a$ führen zunächst Reactionsgleichungen von der Form $M_{1}+\mathrm{HCl} \rightarrow M_{3}$ und $M_{4}+\mathrm{HCl}+2 M_{9} \rightarrow M_{3}$ zu identischen Resultaten. Es genügt daher, eine der beiden Formen zu betrachten.

Das Gleiche gilt für Reactionen von der Form

$$
M_{1}+\mathrm{HCl} \rightarrow M_{3} \text { und } M_{1}+\mathrm{HCl} \rightarrow M_{6}+2 M_{9} .
$$

Denn die Geschwindigkeitsgleichung ist für beide Reactionen wegen der Identität der linken Seiten der vollständigen Reactionsgleichungen dieselbe. Aber auch das gebildete Product ist nach beiden Gleichungen in jedem Augenblicke dasselbe. Denn innerhalb der hylotropen Gruppe des Hydrochlorcinchonins herrscht in jedem Augenblicke Gleichgewicht; die Vertheilung des Hydrochlorcinchonins auf die beiden Molekelarten $M_{3}$ und $M_{6}$ hängt nur von seiner jeweiligen Gesammtconcentration, aber nicht davon $a b$, ob durch eine Reaction primär undissociiertes Salz oder die dazugehörigen Ionen entstehen.

Ferner brauchen aber auch Reactionen von der Form $M_{1}+\mathrm{HCl} \rightarrow M_{3}$ und $M_{4}+\mathrm{HCl} \rightarrow M_{6}$ nicht gesondert betrachtet zu werden, wenn man die Concentration der Chlorionen als unveränderlich ansieht. Letzteres ist zulässig, weil der Halogen-

1 Vergl. Jahn, Z. f. physikal. Chemie, 33, 545 (1900). 
wasserstoff bei den Versuchen in großem Überschusse vorhanden war. Bezeichnet man mit $C_{1}$ die jeweilige Gesammtconcentration des Cinchonins, so ist $C_{1}=A_{1}-\xi_{1}+A_{4}-\xi_{4}$. Ferner ist $A_{1}-\xi_{1}=x_{1}\left(A_{4}-\xi_{4}\right) A_{9}^{2}$, wo $x_{1}$ die Dissociationsconstante des Cinchonindichlorhydrates und $A_{9}$ die Concentration der Chlorionen bedeutet. Die beiden Gleichungen geben

$$
A_{1}-\xi_{1}=C_{1} \frac{x_{1} A_{9}^{2}}{1+x_{1} A_{9}^{2}} \text { und } A_{4}-\xi_{4}=C_{1} \frac{1}{1+x_{1} A_{9}^{2}} .
$$

Man kann daher jede der beiden in Rede stehenden Reactionen sammt den dazugehörigen Dissociationsgleichgewichten ersetzt denken durch die Reaction

$$
\mathrm{Ci}+\mathrm{HCl} \rightarrow \mathrm{C}_{19} \mathrm{H}_{23} \mathrm{ClN}_{2} \mathrm{O} \text {, }
$$

wobei dann die Geschwindigkeitsconstanten $k^{\prime}$ oder $k^{\prime \prime}$ zu ersetzen sind durch die Constanten $\frac{k^{\prime} x_{1} A_{9}^{2}}{1+x_{1} A_{9}^{2}}$ oder $\frac{k^{\prime \prime}}{1+x_{1} A_{9}^{2}}$. Es ist zu beachten, dass diese neuen Constanten von der Concentration des Halogenwasserstoffes abhängen.

Treten beide Reactionen nebeneinander auf, so summieren sie sich zu einer einzigen.

Da es auch gleichgiltig ist, ob in der Reactionsgleichung $\mathrm{HCl}$ oder $\stackrel{+}{\mathrm{H}}+\overline{\mathrm{C}}$ laftritt, können alle möglichen Reactionen, welche Cinchonin in Hydrochlorcinchonin überführen, sammt den dazugehörigen Dissociationsgleichgewichten ersetzt gedacht werden durch die Reaction $\mathrm{Ci}+\mathrm{HCl} \rightarrow \mathrm{C}_{19} \mathrm{H}_{23} \mathrm{ClN}_{2} \mathrm{O}$, in deren Geschwindigkeitsgleichung nur die Gesammtconcentrationen des Cinchonins und Chlorwasserstoffes als Veränderliche eingehen.

Gleiches gilt für die anderen langsam verlaufenden Reactionen. Welche Annahmen man auch über die betheiligten Molekeln machen mag, immer können sie sammt den Dissociationsgleichgewichten ersetzt werden durch Reactionen, in deren Geschwindigkeitsgleichungen nur die Concentrationen der hylotropen Gruppen, aber nicht die der Molekelarten auftreten.

8. 80. Man kann sich daher sämmtliche in dem System vorkommenden Reactionen ersetzt denken durch fünf Reactionen, deren gewöhnliche Reactionsgleichungen sind: 


$$
\begin{aligned}
\text { I. } \mathrm{Ci}+\mathrm{HCl} & \rightarrow \mathrm{C}_{19} \mathrm{H}_{23} \mathrm{ClN}_{2} \mathrm{O} \\
\text { II. } \mathrm{Ci} & \rightarrow \mathrm{Ci}^{\prime} \\
\text { III. } \mathrm{C}_{19} \mathrm{H}_{23} \mathrm{ClN}_{2} \mathrm{O} & \rightarrow \mathrm{Ci}^{\prime}+\mathrm{HCl} \\
\text { IV. } \mathrm{C}_{19} \mathrm{H}_{23}^{\prime} \mathrm{ClN}_{2} \mathrm{O} & \rightarrow \mathrm{Ci}+\mathrm{HCl} \\
\text { V. } \mathrm{Ci}^{\prime} & \rightarrow \mathrm{Ci} .
\end{aligned}
$$

Wie Reaction $\mathrm{Cl}^{\prime}+\mathrm{HCl} \rightarrow \mathrm{C}_{19} \mathrm{H}_{23} \mathrm{ClN}_{2} \mathrm{O}$ kommt nicht in Betracht. Denn aus den Versuchen geht hervor, dass das Umwandlungsverhältnis aufhört constant zu sein, wenn diese Reaction in erheblichem Maße eintritt.

Hält man katalytische Wirkungen der Cinchoninbasen für ausgeschlossen, so kann man über die Form der dazugehörigen vollständigen Reactionsgleichungen Folgendes sagen.

Da die linken Seiten der vollständigen Reactionsgleichungen, deren Anfangsgeschwindigkeit nicht Null ist, llinsichtlich des Chlorwasserstoffes identisch sein müssen, muss bei der Reaction II auf beiden Seiten $\mathrm{HCl}$ (als Katalysator) hinzugefügt werden. Es steht nichts im Wege, außerdem in allen fünf Gleichungen noch weitere HCl-Molekeln oder Wasserstoffoder Chlorionen als Katalysatoren hinzuzufügen; jedoch muss dies bei den Reactionen I und II in gleicher Weise geschehen. Hiedurch würde an den forgenden Auseinandersetzungen nichts geändert werden, da wegen des großen Überschusses des Halogenwasserstoffes die Concentrationen der undissocierten Chlorwasserstoffmolekeln, sowie der Chlor- und Wasserstoffionen als constant betrachtet werden können. Die auf diese Molekelarten bezüglichen Factoren in den Geschwindigkeitsgleichungen vereinigen sich mit den Geschwindigkeitsconstanten.

8. 81. Im folgenden werden die Anfangsconcentrationén des Cinchonins, $\alpha-i$-Cinchonins und Hydrochlorcinchonins mit $A_{1}, A_{2}, A_{3}$, die Concentrationsabnahmen dieser Stoffe mit $\zeta_{1}, \zeta_{2}, \zeta_{3}$, die Geschwindigkeiten der fün Reactionen mit $\frac{d x}{d t}, \frac{d y}{d t}, \frac{d z}{d t}$ $\frac{d u}{d t}, \frac{d v}{d t}$ bezeichnet. $A_{2}$ und $A_{3}$ sind Null.

Für: das Umwandlüngsverhältnis zwischen $\alpha-i$ :Cinchonir und Hydrochlorcinchonin hat man 


$$
\frac{d \zeta_{2}}{d t}: \frac{d \zeta_{3}}{d t}=\frac{-\frac{d y}{d t}-\frac{d z}{d t}+\frac{d v}{d t}}{-\frac{d x}{d t}+\frac{d z}{d t}+\frac{d u}{d t}} .
$$

\$. 82. Die Frage, ob zwischen den fünf fingierten Reactionen des \$. 80 constante Geschwindigkeitsverhältnisse möglich sind, lässt sich nunmehr leicht erledigen.

Das Reactionspaar I, II hat ein constantes Geschwindigkeitsverhältnis wegen del Gleichheit der linken Seiten der vollständigen Reactionsgleichungen. Wenn außerdem die Reactionspaare I, III, I, IV, und II, V constante Geschwindigkeitsverhältnisse aufweisen würden, so würden zwischen allen Reactionen constante Geschwindigkeitsverhältnisse bestehen.

Für das Reactionspaar I, IV gibt Gleichung 13) als nothwendige Bedingung

$$
0=k_{1} A_{1}\left(A_{1}+A_{3}\right)+\left(k_{2}-k_{3}\right) A_{1} A_{3}-k_{4} A_{3}\left(A_{1}+A_{3}\right)-k_{3} A_{2} A_{3}
$$

Für $A_{2}=A_{3}=0$ bleibt $0=k_{1} A_{1}^{2}$ oder $k_{1}=0$. Das ist aber im vorliegenden Falle nicht möglich, da die Reaction I die einzige ist, bei welcher Hydrochlorcinchonin gebildet wird, und da dieser Körper thatsächlich entsteht. Daher ist die Reaction IV ausgeschlossen, wenn das constante Umwandlungsverhältnis auf constanten Geschwindigkeitsverhältnissen beruhen soll.

Es bleiben daher die Reactionen I, II, III und V möglich. Für das Reactionspaar I, III gibt aber die Gleichung 13) ebenfalls die Bedingung $k_{1}=0$. Daher ist auch die Reaction III ausgeschlossen.

Es bleiben nur noch die Reactionen I, II und V. Damit $z w i s c h e n$ den Reactionen II und $V$ ein constantes Geschwindig: keitsverhältnis bestehen kann, ist nach Gleichung 13) nöthig, dass $k_{2}=0$. Dann bleibt keine Reaction übrig, bei der $\alpha-i$-Cinchonin gebildet wird.

Wenn daher das constante Umwandlungsverhältnis auf constanten Geschwindigkeitsverhältnissen beruhen soll, können nur die Reactionen I und II stattfinden. §. 27 gibt daher keine neuen Bedingüngen für das Auftreten des constanten Umwandlungsverhältnisses. 
§. 83. Dagegen kann das Umwandlungsverhältnis zwischen $\alpha-i$-Cinchonin und Hydrochlorcinchonin nach $\S .68$ constant werden. Bei der Untersuchung dieses Falles sollen wieder 'die fingierten Reactionsgleichungen des $\S .80$ zugrunde gelegt werden.

Aus den fünf Reactionsgleichungen können zehn Reactionspaare gebildet werden, welche in Gleichung 29) ebenso viele Glieder geben. Doch werden die auf die Reactionspaare I, II und III, IV bezüglichen Glieder wegen Erfüllung der Gleichung 13) Null. Ferner fallen die Paare von Gegenwirkungen (I, IV und II, V) heraus, weil die Coefficienten von der Form

$$
\left[\left(u_{1}-\alpha_{1}\right)\left(o_{2}-\beta_{2}\right)-\left(n_{2}-\alpha_{2}\right)\left(o_{1}-\beta_{1}\right)\right]
$$

Null werden.

Nach Einführung von $A_{2}=A_{3}=0$ erhält man als nothwendige Bedingung für das Auftreten eines constanten Umwandlungsverhältnisses

$$
k_{5}=\frac{1}{k_{2}}\left(k_{1} k_{3}+k_{2} k_{3}+k_{2} k_{4}\right)
$$

Man sieht wohl, dass der Fall des $\$ .78\left(k_{3}=k_{4}=k_{5}=0\right)$ hierin inbegriffen ist.

Die Bedingung 32) ist aber für das Auftreten eines constanten Umwandlungsverhältnisses zwischen $\alpha-i$-Cinchonin und Hydrochlorcinchonin nicht bloß nothwendig, sondern auch hinreichend, wie die Integration der Geschwindigkeitsgleichungen ergibt.

§. 84. Da fünf Reactionen, aber nur drei Stoffe von veränderlicher Concentration vorkommen, empfiehlt es sich, der Integration nicht die Gleichungen für $\frac{d x}{d t}$ u. s. w., sondern die Gleichungen für $\frac{d \zeta_{1}}{d t}$ u. s. w. zugrunde zu legen.

Für $A_{2}=A_{3}=0$ ist

$$
\frac{d x}{d t}=k_{1}\left(A_{1}-\zeta_{1}\right), \quad \frac{d y}{d t}=k_{2}\left(A_{1}-\zeta_{1}\right), \quad \frac{d z}{d t}=-k_{3} \zeta_{3},
$$


Chemische Kinetik homogener Systeme.

$$
\frac{d u}{d t}=-k_{4} \zeta_{3}, \quad \frac{d v}{d t}=-k_{5} \zeta_{2} .
$$

Daraus folgt (Gleichung 2), §. 4)

$$
\begin{aligned}
& \frac{d \zeta_{1}}{d t}=\left(k_{1}+k_{2}\right)\left(A_{1}-\zeta_{1}\right)+k_{4} \zeta_{3}+k_{5} \zeta_{2} \\
& \frac{d \zeta_{2}}{d t}=-k_{2}\left(A_{1}-\zeta_{1}\right)+k_{3} \zeta_{3}-k_{5} \zeta_{2} \\
& \frac{d \zeta_{3}}{d t}=-k_{1}\left(A_{1}-\zeta_{1}\right)-\left(k_{3}+k_{4}\right) \zeta_{3}
\end{aligned}
$$

und wegen

$$
\begin{aligned}
& \frac{d \zeta_{1}}{d t}=-\left(\frac{d \zeta_{2}}{d t}+\frac{d \zeta_{3}}{d t}\right), \quad \zeta_{1}=-\left(\zeta_{2}+\zeta_{3}\right) \\
& \frac{d \zeta_{2}}{d t}=-k_{2} A_{1}-\left(k_{2}+k_{5}\right) \zeta_{2}+\left(k_{3}-k_{2}\right) \zeta_{3} \\
& \frac{d \zeta_{3}}{d t}=-k_{1} A_{1}-k_{1} \zeta_{2}-\left(k_{1}+k_{3}+k_{4}\right) \zeta_{3} .
\end{aligned}
$$

Setzt man

$$
\begin{aligned}
\mathbf{\Sigma} k & =k_{1}+k_{2}+k_{3}+k_{4}+k_{5} \\
K & =\left(k_{2}+k_{5}\right)\left(k_{1}+k_{3}+k_{4}\right)+k_{1}\left(k_{3}-k_{2}\right) \\
\vartheta^{\prime} & =\frac{\mathbf{\Sigma} k}{2}+\sqrt{\left(\frac{\mathbf{\Sigma} k}{2}\right)^{2}-K} \\
\vartheta^{\prime \prime} & =\frac{\mathbf{\Sigma} k}{2}-\sqrt{\left(\frac{\mathbf{\Sigma} k}{2}\right)^{2}-K} \\
\lambda^{\prime} & =\frac{k_{3}-k_{2}}{k_{1}+k_{3}+k_{4}-\vartheta^{\prime}} \\
\lambda^{\prime \prime} & =\frac{k_{3}-k_{2}-\vartheta^{\prime \prime}}{k_{1}+k_{3}+k_{4}-\vartheta^{\prime \prime}}
\end{aligned}
$$


so wird

$$
\begin{aligned}
& \zeta_{2}=\frac{A_{1}}{\lambda^{\prime}-\lambda^{\prime \prime}}\left\{-\frac{\lambda^{\prime \prime}\left(k_{1} \lambda^{\prime}+k_{2}\right)}{\vartheta^{\prime}}\left(e^{-\vartheta^{\prime} t}-1\right)+\frac{\lambda^{\prime}\left(k_{1} \lambda^{\prime \prime}+k_{2}\right)}{\vartheta^{\prime \prime}}\left(e^{-\vartheta^{\prime \prime} t}-1\right)\right\} \\
& \zeta_{3}=\frac{A_{1}}{\lambda^{\prime}-\lambda^{\prime \prime}}\left\{\frac{k_{1} \lambda^{\prime}+k_{2}}{\vartheta^{\prime}}\left(e^{-\vartheta^{\prime} t}-1\right)-\frac{k_{1} \lambda^{\prime \prime}+k_{2}}{\vartheta^{\prime \prime}}\left(e^{-\vartheta^{\prime \prime} t}-1\right)\right\} .
\end{aligned}
$$

Hieraus folgt

$$
\begin{aligned}
& \frac{d \zeta_{2}}{d t}=\frac{A_{1}}{\lambda^{\prime}-\lambda^{\prime \prime}}\left\{\lambda^{\prime \prime}\left(k_{1} \lambda^{\prime}+k_{2}\right) e^{-\vartheta^{\prime} t}-\lambda^{\prime}\left(k_{1} \lambda^{\prime \prime}+k_{2}\right) e^{-\vartheta^{\prime \prime} t}\right\} \\
& \frac{d \zeta_{3}}{d t}=\frac{A_{1}}{\lambda^{\prime}-\lambda^{\prime \prime}}\left\{-\left(k_{1} \lambda^{\prime}+k_{2}\right) e^{-v^{\prime} t}+\left(k_{1} \lambda^{\prime \prime}+k_{2}\right) e^{-\vartheta^{\prime \prime} t}\right\}
\end{aligned}
$$

und

$$
\begin{aligned}
& \frac{d \zeta_{2}}{d t}: \frac{d \zeta_{3}}{d t}=\frac{\lambda^{\prime \prime}\left(k_{1} \lambda^{\prime}+k_{2}\right)-\lambda^{\prime}\left(k_{1} \lambda^{\prime \prime}+k_{2}\right) e^{\left(\vartheta^{\prime}-\vartheta^{\prime \prime}\right) t}}{-\left(k_{1} \lambda^{\prime}+k_{2}\right)+\left(k_{1} \lambda^{\prime \prime}+k_{2}\right) e^{\left(\vartheta^{\prime}-\vartheta^{\prime \prime}\right) t}} \\
& =\frac{-\left[k_{1} k_{3}+k_{2}\left(k_{3}+k_{4}-\vartheta^{\prime}\right)\right]+\left[k_{1} k_{3}+k_{2}\left(k_{3}+k_{4}-\vartheta^{\prime \prime}\right)\right] e^{2 t} \sqrt{\left(\frac{\sum k}{2}\right)^{2}-K}}{k_{1}\left[\left(k_{1}+k_{2}+k_{3}+k_{4}-\vartheta^{\prime \prime}\right)-\left(k_{1}+k_{2}+k_{3}+k_{4}-\vartheta^{\prime}\right) e^{2 i \sqrt{\left(\frac{2 k}{2}\right)^{2}-K}}\right.} .
\end{aligned}
$$

Dieser Ausdruck wird constant für $k_{1} \lambda^{\prime}+k_{2}=0$ oder für $k_{1} \lambda^{\prime \prime}+k_{2}=0$. Setzt man die Werte von $\lambda^{\prime}$ und $\lambda^{\prime \prime}$ ein und rechnet $k_{5}$ aus, so findet man in beiden Fällen

$$
k_{5}=\frac{1}{k_{2}}\left(k_{1} k_{3}+k_{2} k_{3}+k_{2} k_{4}\right)
$$

Das stimmt mit Gleichung 32) überein, welche also nicht bloß die nothwendige, sondern auch die hinreichende Bedingung für das Auftreten eines constanten Umwandlungsverhältnisses ist. In diesem Falle stehen die Reactionen III bis. V untereinander (aber nicht mit I und II) in einem constanten Geschwindigkeitsverhältnisse. In der That liefert auch Gleichung 13) dieselbe Bedingung für die Constanz des Geschwindigkeitsverhältnisses zwischen den Reactionen III und $V$, wenn man berücksichtigt, $\operatorname{dass} A_{3}=\frac{k_{1}}{k_{2}} A_{2}$ ist. 
Bei oberflächlicher Betrachtung der Gleichung für $\frac{d \zeta_{2}}{d t}: \frac{d \zeta_{0}}{d t}$ könnte man glauben, dass das Umwandlungsverhältnis auch für $\vartheta^{\prime}=\vartheta^{\prime \prime}$ constant wird. Aber dann wird auch $\lambda^{\prime}=\lambda^{\prime \prime}$ und $\frac{d \zeta_{2}}{d t}: \frac{d \zeta_{3}}{d t}=\frac{0}{0}$. Die Auswertung dieses Ausdruckes (etwa indem man Zähler und Nenner nach $k_{\tilde{5}}$ differenziert) gibt

$$
\frac{d \zeta_{2}}{d t}: \frac{d \zeta_{3}}{d t}=\frac{k_{2} \lambda+\left(k_{3}-k_{2}\right)\left(k_{1} \lambda+k_{2}\right) t}{k_{1} \lambda-\left(k_{1}+k_{3}+k_{4}-\frac{\Sigma}{2}\right)\left(k_{1} \lambda+k_{2}\right) t},
$$

welcher Ausdruck nur für $k_{1} \lambda+k_{2}=0$ constant wird.

\&. 85. Führt man die Bedingung $k_{5}=\frac{k_{1} k_{3}}{k_{2}}+k_{3}+k_{4}$ in Gleichung 33) ein, so erhält man wegen

$$
\begin{gathered}
\frac{\Sigma k}{2}=\frac{k_{1}+k_{2}}{2}+k_{3}+k_{4}+\frac{k_{1} k_{3}}{2 k_{2}}, \quad \sqrt{\left(\frac{\Sigma}{2}\right)^{2}-K}=\frac{k_{1}+k_{2}}{2}-\frac{k_{1} k_{3}}{2 k_{2}} \\
\vartheta^{\prime}=k_{1}+k_{2}+k_{3}+k_{4}, \quad \vartheta^{\prime \prime}=k_{3}+k_{4}+\frac{k_{1} k_{3}}{k_{2}} \\
\lambda^{\prime}=-\frac{k_{3}-k_{2}}{k_{2}}, \quad \lambda^{\prime \prime}=-\frac{k_{2}}{k_{1}}: \\
\frac{d \zeta_{2}}{d \zeta_{3}}=\frac{k_{2}}{k_{1}}
\end{gathered}
$$

Das Umwandlungsverhältnis ist also dasselbe, als wenn nur die Reactionen I und II stattfinden würden. Das entspricht dem Umstande, dass die Reactionen III bis $\mathrm{V}$ die Anfangsgeschwindigkeit Null haben.

§. 86. Das experimentell gefundene Umwandlungsverhältnis, welches im folgenden mit $a$ bezeichnet werden soll, ist von der Säureconcentration unabhängig. Es ist $a=\frac{k_{2}}{k_{1}}$. Nun sind aber $k_{1}$ und $k_{2}$ ihrer Bedeutung nach von der Säureconcentration abhängig (\$.79). Es können daher die den fingierten Reactionen I und II entsprechenden wirklichen Reactionen nicht beliebig gewählt werden. 
Die $k$ haben im allgemeinen die Form

$$
k=\frac{k^{\prime} x A_{9}^{2}}{1+x A_{9}^{2}} A_{7}^{n_{2}} A_{8}^{n_{\mathrm{s}}} A_{9}^{n_{9}}+\frac{k^{\prime \prime}}{1+x A_{9}^{2}} A_{\mathrm{\gamma}}^{o_{7}} A_{8}^{o_{\mathrm{s}}} A_{9}^{o_{9}} .
$$

$n_{7}, n_{8}, n_{9}, o_{7}, o_{8}, o_{9}$ bedeuten die Molekelzahlen der Molekelarten $M_{7}, M_{8}$ und $M_{9}$ auf den linken Seiten der wirklichen (nicht der fingierten) Reactionsgleichungen.

daher nur eintreten:

Unabhängigkeit des $\frac{k_{1}}{k_{2}}$ von der Säureconcentration kann

1. wenn bei den den fingierten Reactionen I und II entsprechenden wirklichen Reactionen entweder nur die Molekeln $M_{1}$ oder nur die Molekeln $M_{4}$ aufgebraucht werden und die linken Seiten der beiden vollständigen Reactionsgleichungen auch hinsichtlich des Halogenwasserstoffes identisch sind (nur Ersatz von $\mathrm{HCl}$ durch $\stackrel{+}{\mathrm{H}}+\overrightarrow{\mathrm{C}}$ ist nach $\S .6$ a zulässig);

2. wenn den fingierten Reactionen wirkliche Reactionen entsprechen, bei denen theils $M_{1}$, theils $M_{4}$ aufgebraucht wird, und wenn außerdem $A_{7}^{n_{7}+2} A_{8}^{n_{s}+2} A_{9}^{n_{9}+2}=A_{7}^{o_{7}} A_{8}^{o_{8}} A_{9}^{o_{9}}=\ldots$ ist. Ein Beispiel für letzteren Fall ist

$$
M_{1}+\mathrm{HCl} \rightarrow M_{3} \text { und } M_{4}+2 \overline{\mathrm{Cl}}+\mathrm{HCl} \rightarrow M_{2}+\mathrm{HCl} .
$$

Auch

$$
\begin{gathered}
M_{4}+2 \overline{\mathrm{Cl}}+\mathrm{HCl} \rightarrow M_{3}, \quad M_{1}+\mathrm{HCl} \rightarrow M_{2}+\mathrm{HCl}, \\
M_{4}+2 \overline{\mathrm{Cl}}+\mathrm{HCl} \rightarrow M_{5}+2 \overline{\mathrm{Cl}}+\mathrm{HCl}
\end{gathered}
$$

können neben den beiden ersten Reactionen stattfinden.

Es ist daher auch im Falle des constanten Umwandlungsverhältnisses nach $\$ .68$ für die den fingierten Reactionen I und Il entsprechenden wirklichen Reactionen, deren Zahl größer als zwei sein kann, Gleichheit der linken Seiten der vollständigen Reactionsgleichungen (mit der dem $\S .6 a$ entsprechenden Einschränkung) erforderlich.

§. 87. Außerdem besteht eine Bedingung für die Geschwindigkeitsconstanten der anderen drei fingierten Reactionen. Ersetzt man nämlich in Gleichung 32) $k_{2}$ durch $a k_{1}$, so geht sie 
über in $k_{5}=k_{3}\left(1+\frac{1}{a}\right)+k_{4}$. Auch diese Gleichung muss bei wechselnden Säureconcentrationen richtig bleiben. Hiebei ist zu beachten, dass die $k$ die im vorigen Paragraphen angegebene Form haben, also möglicherweise Summen von zwei Gliedern sein können, und dass in $k_{3}$ und $k_{4} x_{3}$, dagegen in $k_{5} x_{2}$ auftritt. Es ist wieder erforderlich, dass die in den Summanden von $k_{3}, k_{4}$ und $k_{5}$ auftretenden Säurefunctionen identisch sind; dazu gehört insbesondere auch die Gleichheit von $x_{2}$ und $x_{3}$. Die Gleichheit der Dissociationsconstanten der Chlorhydrate von $\alpha-i$-Cinchonin und Hydrochlorcinchonin wird wohl in Wirklichkeit annähernd zutreffen.

Die Gleichung 32) ist also beispielsweise erfüllbar für die Reactionen

$$
M_{3} \rightarrow M_{2}+\mathrm{HCl}, M_{3} \rightarrow M_{1}+\mathrm{HCl}, M_{2} \rightarrow M_{1},
$$

zu welchen noch etwa hinzutreten können

$$
M_{6}+2 M_{9} \rightarrow M_{2}+\mathrm{HCl}, M_{6}+2 M_{9} \rightarrow M_{5}+2 M_{9}+\mathrm{HCl} \text { u. s.w. }
$$

Dagegen können nicht neben den vorerwähnten die Reactionen $M_{3}+\mathrm{HCl} \rightarrow M_{2}+2 \mathrm{HCl}$ oder $M_{6} \rightarrow M_{5}+\mathrm{HCl}$ stattfinden. Es ist also (unter Berücksichtigung der aus $\S .6 a$ folgenden Einschränkung) Gleichheit der linken Seiten der vollständigen Reactionsgleichungen der Reactionen III bis $\mathrm{V}$ hinsichtlich des Chlorwasserstoffes nöthig.

§. 88. Es ist schließlich von Interesse, zu untersuchen, ob einzelne der fünf fingierten Reactionen wegbleiben können, ohne dass das constante Umwandlungsverhältnis unmöglich wird.

Dass $k_{3}=k_{4}=k_{5}=0$ mit dem constanten Umwandlungsverhältnisse, beziehungsweise mit Gleichung 32) verträglich ist, ist bereits erwähnt worden.

$k_{1}$ kann nicht Null werden, da sonst $\alpha$ - $i$-Cinchonin überhaupt.nicht gebildet würde.

$k_{2}$ kann ebenfalls nicht Null werden; denn in diesem Falle müsste nach Gleichung 32) auch $k_{3}=0$ sein. Dann wäre aber die Bildung von $\alpha-i$.Cinchonin unmöglich. 
Die Reactionen I und II, also die Bildung von Hydrochlorcinchonin durch directe Anlagerung von Chlorwasserstoff an Cinchonin und dic directe Umlagerung des Cinchonins in a- $i$-Cinchonin sind also für das Zustandekommen des constanten Umwandlungsverhältnisses unentbehrlich.

Dagegen kann $k_{3}$ Null werden; dann ist $k_{5}=k_{4}$. Wenn also $k_{3}=0$ ist, so müssen die Reactionen IV und $V$ entweder beide stattfinden oder beide ausbleiben.

Für $k_{4}=0$ wird $k_{2} k_{\tilde{z}}=\left(k_{1}+k_{2}\right) k_{3}$; es müssen also in diesem Falle die Reactionen III und $V$ beide stattfinden oder beide ausbleiben.

$k_{5}=0$ ist nicht möglich, wenn nicht gleichzeitig $k_{3}=k_{4}=0$ ist. Die $k$ sind ja wesentlich positive Größen. (Von katalytischen Verzögerungen in homogener Lösung ist bei der Betrachtung der Cinchoninumlagerung abgesehen worden.)

Neben den Reactionen I und II können daher nur die Reactionen III, IV und V oder IV und V (die beiden Gegenreactionen) oder III und $V$ oder gar keine andere stattfinden Insbesondere kann die Reaction III, welche der Annahme entspricht, dass das Hydrochlorcinchonin bei der Bildung von $\alpha-i$-Cinchonin aus Cinchonin als $Z_{w}$ ischenproduct auftritt, nur stattfinden, wenn gleichzeitig direct. Umlagerung von Cinchonin in $\alpha-i$-Cinchonin und umgekehrt stattfindet.

§. 89. Als Schlussresultat ergibt sich, dass für das constante Umwandlungsverhältnis die directe Bildung von Hydrochlorcinchonin und $\alpha-i$-Cinchonin aus Cinchonin nothwendig und maßgebend sind. Daneben können allerdings noch die beiden Gegenreactionen und die Umwandlung von Hydrochlorcinchonin in $\alpha$-i-Cinchonin stattfinden, aber nur, wenn deren Geschwindigkeitscoefficienten in bestimmten, von der Natur des Halogens abhängigen Zahlenbeziehungen stehen. Warum diese Coefficienten untẹr den unzähligen möglichen Werten gerade diese bestimmten, zum constanten Umwandlungsverhältnisse führenden annehmen, bedarf eigentlich einer besonderen Erklärung, wenn man das Stattfinden dieser accessorischen Reactionen überhaupt annehmen will. Es ist daher das natïrlichste, anzunehmen, dass nur die für das constante Umwandlungsverhältnis nothwendigen zwei Reactionen statt- 
finden. Diese Annahme entspricht dem in der Naturforschung herrschenden Grundsatze, die einfachste Darstellung der Erscheinungen vorzuziehen, solange nicht die Thatsachen zwingen, sie aufzugeben. Demgemäß können die Schlüsse, die ich in der früher erwähnten Abhandlung über die Cinchoninumlagerung gezogen habe, bis auf weiteres vollinhaltlich aufrecht erhalten werden.

\section{Reactionsgeschwindigkeit bei veränderlichem Volum.}

§. 90. Soviel mir bekannt, hat man bisher die Reactionsgeschwindigkeiten lediglich bei constantem Volum betrachtet.

Bei Reactionen in flüssigen und festen Phasen kann man das Volum in allen Fällen als constant ansehen, da die durch Reactionen hervorgebrachten Volumänderungen unbedeutend sind.

Dagegen sind bei isothermen Gasreactionen bedeutende Volumänderungen möglich. Nichtsdestoweniger sind auch bei Gasen die Reactionsgeschwindigkeiten nur bei constantem Volum betrachtet worden, entsprechend dem Umstande, dass diesbezügliche Versuche meist im geschlossenen Gefäße ausgeführt werden. Die in der Natur und in der Technik vorkommenden Gasreactionen erfolgen dagegen in der Regel nicht bei constantem Volum, sondern bei annähernd constantem Drucke; auch sind einzelne experimentelle Untersuchungen bei veränderlichem Volum ausgeführt worden. Es ist daher die Aufstellung der Gesetze für den Reactionsablauf in Systemen von veränderlichem Volum von Interesse.

§. 91. Auf das chemische Gleichgewicht hat selbsverständlich nur das Endvolum Einfluss, aber nicht die während der Einstellung des Gleichgewichtes auftretenden Volumänderungen. Dagegen wird der Reactionsablauf im allgemeinen durch Volumänderungen beeinflusst.

§. 92. Bei veränderlichem Volum kann die Änderung der Concentration in der Zeiteinheit nicht als Maß der Reactionsgeschwindigkeit betrachtet werden. Denn die Concentrationsänderung rührt nur zum. Theile von der Änderung der Menge des betrachteten Stoffes infolge der Reactionen, zum Theile aber von der Volumänderung her; in einem Systeme von 
veränderlichem Volum treten Concentrationsänderungen auch dann auf, wenn überhaupt keine Reaction stattfindet.

Als Maß für die Reactionsgeschwindigkeit ist daher im allgemeinen die Änderung der Menge des betrachteten Stoffes in der Zeiteinheit in einer gegebenen Masse des reagierenden Systems zu betrachten. Nur bei constantem Volum stellt die Raumeinheit zugleich eine gegebene unveränderliche Masse vor; in diesem speciellen Falle ist es gleichgiltig, ob man die Reactionsgeschwindigkeiten als Mengenänderungen in einer gegebenen Masse oder in einem gegebenen Volum (der Volumeinheit) definiert.

§. 93. Die Grundformel der chemischen Kinetik

$$
\frac{d x}{d t}=k\left(A_{1}-\xi_{1}\right)^{n_{1}}\left(A_{2}-\xi_{2}\right)^{n_{2}} \ldots,
$$

in welcher die Buchstaben wieder dieselbe Bedeutung haben wie in $\S .6$, bezieht sich auf constantes Volum, wie aus ihrer molecular-kinetischen Begründung unmittelbar hervorgeht.

Es sei $\mu$ die zur Zeit $t$ im ganzen Systeme umgesetzte Menge in Grammolekeln, wobei wieder die auf einer Seite der Reactionsgleichung stehenden Stoffe wie eine Molekel gerechnet werden sollen, und $V$ das Volum des Systemes.

Dann ist $\frac{d \mu}{d t}$ die Reactionsgeschwindigkeit.

Die Änderung von $\mu$ in den Zeittheilchen $d t$ ist nun proportional der Zeit, der in der Volumeinheit in der Zeiteinheit eintretenden Umsetzung und dem Volum. Also $d \mu=\frac{d x}{d t} \cdot V d t$. Die Volumänderung $d V$ tritt in die Formel nicht ein.

Anders geschrieben:

$$
\frac{1}{V} \cdot \frac{d \mu}{d t}=\frac{d x}{d t}
$$

Bei Ableitung dieser Formel ist constantes Volum nicht vorausgesetzt; sie gilt also allgemein. Da $\mu=V x$, so ist

$$
\frac{d x}{d t}=\frac{d \frac{\mu}{V}}{d t} .
$$


Daraus ist unmittelbar ersichtlich, dass in $\frac{d x}{d t}$ das Volum als constant $z u$ betrachten ist.

\$. 94. Bezeichnet man die im reagierenden Systeme zur Zeit $t$ enthaltene Menge des Stoffes $M_{1}$ mit $\mu_{1}$, die des Stoffes $M_{2}$ mit $\mu_{2}$ u. s. w., so ist

$$
A_{1}-\xi_{1}=\frac{\mu_{1}}{V}, \quad A_{2}-\xi_{2}=\frac{\mu_{2}}{V} \text { u. s.w. }
$$

Daher nimmt die Gleichung für die Reactionsgeschwindigkeit die Form an

$$
\frac{d \mu}{d t}=V \frac{d x}{d t}=\frac{k \mu_{1}^{n_{1}} \mu_{2}^{n_{2}} \ldots}{V^{n_{1}+n_{2}+\cdots-1}} .
$$

Diese Gleichung für die Reactionsgeschwindigkeit gilt ganz allgemein. Bei veränderlichem Volum ist das Volum als Function der jeweiligen Zusammensetzung des Systemes oder als Function der Zeit einzusetzen. Bei veränderlicher Temperatur ist auch $k$ als Function der Temperatur und letztere als Function der Zeit einzuführen.

Man kann die Gleichung 34) selbstverständlich auch bei constantem Volum benützen; das ist in der That in einem einfachen speciellen Falle (bei der Esterbildung) bereits durch van t'Hoff' ${ }^{1}$ geschehen.

Die Geschwindigkeitsconstante ist von der Masse des reagierenden Systemes unabhängig, wie aus Gleichung 34) unmittelbar hervorgeht.

§. 95. Bei monomolecularen Reactionen $\left(n_{1}=1, n_{2}=\ldots\right.$ $\ldots=0$ ) fällt das Volum aus der Gleichung 34) heraus. Die Reactionsgeschwindigkeit ist also unabhängig von beliebigen während der Reaction stattfindenden Volumänderungen. Das entspricht dem bekannten Satze, dass die Reactionsgeschwindigkeit dieselbe ist, wenn die Reaction bei verschiedenen, aber während der Reactionsdauer constanten Volumen vorgenommen wird.

1 Ber. der Deutschen chem. Ges., 10, 671 (1877). 
§. 96. Einen speciellen Fall der Reactionen bei veränderlichem Volum bilden die Gasreactionen bei constantem Drucke. Unter Voraussetzung der idealen Gasgesetze lässt sich die Gleichung 34) in folgender Weise umformen.

Das Volum einer Grammolekel ist in diesem Falle gegeben durch $\frac{R T}{p}$, wo $R$ die Gasconstante, $T$ die absolute Temperatur, $p$ den (constanten) Druck bedeutet.

Es sei $N$ die Zahl der beim Umsatze der in der Reactionsgleichung stehenden Mengen verschwindenden Grammolekel $N_{1}$ die Zahl der entstehenden Grammolekel.

Beim Umsatze der in der Reactionsgleichung stehenden Quantitäten $(\mu=1)$ vermehrt sich also das Volum um $\left(N_{1}-N\right) \frac{R T}{p}$, beim Umsatze $\mu$ um $\left(N_{1}-N\right) \frac{R T}{p} \mu$. Ist das Anfangsvolum (für $t=0, \mu=0$ ) $V_{0}$, so ist das Volum zur Zeit $t$ gegeben durch

$$
V=V_{0}+\left(N_{1}-N\right) \frac{R T}{p} \mu .
$$

Drückt man $N_{1}-N$ durch die in der Reactionsgleichung auftretenden Coefficienten aus, so erhält man

$$
N_{1}-N=\alpha_{1}+\alpha_{2}+\ldots-\left(n_{1}+n_{2}+\ldots\right) .
$$

Die Formel für die Reactionsgeschwindigkeit in Gasen bei constantem Drucke wird daher

$$
\frac{d \mu}{d t}=\frac{k \mu_{1}^{n_{1}} \mu_{2}^{n_{2}} \ldots}{\left[V_{0}+\left(N_{1}-N\right) \frac{R T}{p} \mu\right]^{n_{1}+t_{2}+\cdots-1}} .
$$

\$. 97. Aus Gleichung 35) folgt, dass für $N_{1}=N \quad V=V_{0}$ ist. Verlaufen Gasreactionen ohne Änderung der Molekelzahl, so ist bei constantem Drucke auch das Volum constant.

Der Verlauf der Gasreactionen bei constantem Drucke unterscheidet sich daher von dem Verlaufe bei constantem Volum nur, wenn die Reactionen mit Änderung der Molekelzahl verlaufen und außerdem nicht monomolecular sind (\$.95). Nur in diesem Falle ist daher bei constantem Drucke die 
Anwendung der Gleichung 36) nothwendig. Bei monomolecularen Reactionen und bei Reactionen ohne Änderung der Molekelzahl kann auch bei constantem Drucke der Reactionsablauf nach der für constantes Volum giltigen Formel berechnet werden.

§. 98. Eine Reaction, deren Verlauf bei constantem Drucke nach Gleichung 36) berechinet werden muss, ist die Vereinigung des Knallgases zu Wasserdampf. Findet die Reaction nach der Gleichung $2 \mathrm{H}_{2}+\mathrm{O}_{2} \rightarrow 2 \mathrm{H}_{2} \mathrm{O}$ statt, so ist $u_{1}=2$, $n_{2}=1, \alpha_{3}=2$, wenn die Indices $1,2,3$ sich der Reihe nach auf $\mathrm{H}_{2}, \mathrm{O}_{2}$ und $\mathrm{H}_{2} \mathrm{O}$ beziehen. Dann geht Gleichung 36) über in

$$
\frac{d \mu}{d t}=\frac{k \mu_{1}^{2} \mu_{2}}{\left(V_{0}-\frac{R T}{p} \mu\right)^{2}}=\frac{k\left(\mathrm{M}_{1}-2 \mu\right)^{2}\left(\mathrm{M}_{2}-\mu\right)}{\left(V_{0}-\frac{R T}{p} \mu\right)^{2}},
$$

wenn $\mathbf{M}_{1}$ die Anfangsmenge des Wasserstoffes, $\mathbf{M}_{2}$ die Anfangsmenge des Sauerstoffes (in Grammolekeln) bedeutet. $V_{0}$ ist $\frac{R T}{p}\left(\mathbf{M}_{1}+\mathbf{M}_{2}\right)$.

Sind Wasserstoff und Sauerstoff in äquivalenten Mengen vorhanden $\left(\mathbf{M}_{1}=2 \mathbf{M}_{2}\right)$, so wird $V_{0}=\frac{3 R T \mathbf{M}_{2}}{p}$ und

$$
\left(\frac{d \mu}{d t}\right)_{p}=\frac{4 k p^{2}}{R^{2} T^{2}} \cdot \frac{\left(\mathrm{M}_{2}-\mu\right)^{3}}{\left(3 \mathrm{M}_{2}-\mu\right)^{2}}
$$

Durch Integration erhält man, wenn man gleichzeitig die umgesetzte Menge in Bruchtheilen der Anfangsmenge $\left(x=\frac{\mu}{M_{2}}\right)$ einführt:

$$
\frac{4 k p^{2}}{R^{2} T^{2}}=\frac{1}{t}\left\{\frac{8 x-6 x^{2}}{(1-x)^{2}}-\operatorname{lognat}(1-x)\right\}
$$

Dagegen hat man bei constantem Volum und äquivalenten Mengen, wenn der Anfangsdruck $p_{0}$ ist

$$
\left(\frac{d \mu}{d t}\right)_{V}=\frac{4 k\left(\mathrm{M}_{2}-\mu\right)^{3}}{V_{0}^{2}}=\frac{4 k p_{0}^{2}\left(\mathrm{M}_{2}-\mu_{1}\right)^{3}}{9 R^{2} T^{2} \mathrm{M}_{2}^{2}}
$$


Daraus folgt durch Integration

$$
\frac{4 k p_{0}^{2}}{R^{2} T^{2}}=\frac{1}{t} \cdot \frac{9 x-4 \cdot 5 x^{2}}{(1-x)^{2}} .
$$

Aus den Geschwindigkeitsgleichungen sieht man, dass die Reaction bei constantem Volum langsamer verläuft als bei constantem Drucke. Für $t=0$ sind die Ausdrücke identisch. Dann aber bleibt bei constantem Volum der Nenner unverändert, während er bei constantem Drucke fortwährend sinkt. Das entspricht dem Umstande, dass bei constantem Volum der Druck fortwährend sinkt.

Der Unterschied zwischen beiden Formeln wird um so größer, je weiter die Reaction fortschreitet. Bei kleinen Umsetzungen bleiben die Unterschiede klein.

§. 99. Ausgedehnte Versuchsreihen über die Geschwindigkeit der Knallgasvereinigung bei constantem Drucke sind in einer schönen Arbeit von Bodenste in ${ }^{1}$ enthalten. Bodenstein hat seine Versuche so berechnet, wie wenn sie bei constantem Volum verlaufen wären; die Constanz der Geschwindigkeitscoefficienten war nicht besonders befriedigend, was Bodenstein dem bei dieser Reaction bekanntlich besonders großen Einflusse der Störungen zuschrieb. Indes zeigt eine von mir vorgenommene theilweise Neuberechnung der Constanten, dass die unbefriedigende Constanz zum Theile der principiell unrichtigen Berechnung Bodensteins zur Last fällt. Es sei jedoch gleich hervorgehoben, dass die wesentlichen Resultate der Bodenstein'schen Arbeit hiedurch nicht berührt werden.

Dass die Versuche mit erheblichen Störungen behaftet sind, hebt Bodenstein mit Recht hervor; das geht direct aus den Versuchszahlen hervor. Die umgesetzte Menge wurde z. B. in derselben Versuchsreihe (Tabelle 2, Rohr I) zur Zeit 50.19 mit 0.160 , dagegen zur Zeit 61.62 nur mit 0.120 gefunden.

\$. 100. Erheblich bessere Constanten als die Bodensteinsche Berechnung gibt die Formel 37, beziehungsweise 38 bei den Versuchen mit Rohr II und äquivalenten Mengen. Ich stelle

1 Zeitschr. f. phys. Chemie, 29, 665 (1899). Die von mir berechneten Versuche befinden sich auf S. 681, 682, 692, 694 und 696 . 
im folgenden die von Bodenstein und von mir berechneten Constanten zusammen.

Versuchsreihe Tabelle 1, Rohr II $\left(482^{\circ}\right)$.

$\begin{array}{llllll}\text { Zeit } \ldots \ldots \ldots 20.8 & 34.0 & 48.4 & 62.9 & 79.3 & 96.0 \\ \begin{array}{l}k \text { nach Boden- } \\ \text { stein..... } 0.339\end{array} & 0.288 & 0.262 & 0.261 & 0.244 & 0.264 \\ \frac{4 k p^{2}}{R^{2} T^{25}} \text { nach } & & & & & \end{array}$

$\begin{array}{llllllll}\text { Formel 38) } \ldots & 0.0418 & 0 & 0351 & 0.0316 & 0.0312 & 0.0288 & 0.0306\end{array}$

Bei dieser Versuchsreihe stimmen beide Formeln ungefähr gleich schlecht; bei der ersten Beobachtung stimmt sogar Formel 38) schlechter. Indes ist diese Versuchsreihe zur Prüfung des Unterschiedes der beiden Formeln wenig geeignet, da der größte erzielte Umsatz nur $23 \cdot 64 \%$ betrug.

Versuchsreihe Tabelle 2, Rohr II $\left(509^{\circ}\right)$.

Zeit $\ldots \ldots .8 \cdot 82 \quad 19 \cdot 01,31 \cdot 81 \quad 46 \cdot 87 \quad 63 \cdot 40 \quad 77 \cdot 10 \quad 100 \cdot 17$

$k$ nach Boden-

$\begin{array}{lllllll}\text { stein....1.06 } & 1.18 & 1.23 & 1.27 & 1.48 & 1.25 & 1.36\end{array}$

$\frac{4 k p^{2}}{R^{2} T^{2}}$ nach

$\begin{array}{lllllll}\text { Formel 38) } .0 .118 & 0.126 & 0.126 & 0.125 & 0.139 & 0.117 & 0.122\end{array}$

Der höchste Umsatz betrug $52 \cdot 8 \%$. Hier zeigt sich Formel 38) entschieden überlegen. Sieht man von der offenbar mit einem Fehler behafteten Beobachtung für $t=63.40 \mathrm{ab}$, so stimmen die Constanten recht gut, während sie nach der Bodenstein'schen Berechnung erheblich schlechter stimmen und einen ausgesprochen aufsteigenden Gang zeigen.

Versuchsreihe Tabelle $4 A$, Rohr II $\left(572^{\circ}\right)$.

\begin{tabular}{|c|c|c|c|c|c|c|}
\hline Zeit ....... 0.99 & $1 \cdot 60$ & $5 \cdot 16$ & $14 \cdot 70$ & $22 \cdot 82$ & $26 \cdot 09$ & $30 \cdot 50$ \\
\hline $\begin{array}{l}k \text { nach Boden- } \\
\text { stein......10.61 }\end{array}$ & $9 \cdot 5$ & $11 \cdot 9$ & $14 \cdot 9$ & $21 \cdot 9$ & $17 \cdot 1$ & $16 \cdot 3$ \\
\hline $4 k p^{2}$ & & & & & & \\
\hline $2 T^{2}$ & & & & & & \\
\hline Formel 38). . & 0.91 & $1 \cdot 02$ & $1 \cdot 13$ & $1 \cdot 48$ & $1 \cdot 18$ & $1 \cdot 1$ \\
\hline
\end{tabular}

1 In der Abhandlung steht irrthümlich $14 \cdot 2$. 
Versuchsreihe Tabelle 10 , Rohr II $\left(600^{\circ}\right)$.

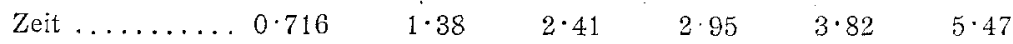

$k$ nach Boden-

$\begin{array}{llllll}\text { stein.....17.0 } & 16 \cdot 7 & 16 \cdot 2 & 16 \cdot 7 & 18 \cdot 6 & 18 \cdot 8\end{array}$

$\frac{4 k p^{2}}{R^{2} T^{2}}$ nach

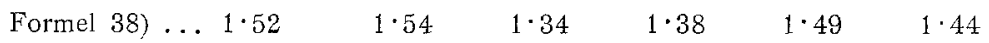

Versuchsreihe Tabelle 6 , Rohr II $\left(638^{\circ}\right)$.

$\begin{array}{llllllll}\text { Zeit } \ldots \ldots \ldots & \ldots .39 & 0.55 & 0.84 & 1.08 & 1.49 & 1.72 & 1.73\end{array}$

$k$ nach Boden-

$\begin{array}{lllllll}\text { stein.....59.9 } 65 \cdot 9 & 60 \cdot 7 & 65 \cdot 0 & 61 \cdot 9 & 68 \cdot 0 & 63 \cdot 3\end{array}$

$\frac{4 k p^{2}}{R^{2} T^{2}}$ nach

Formel 38) $\ldots 4.89 \quad .5 \cdot 30 \quad 4 \cdot 79 \quad 5.02 \quad 4.63 \quad 4.98 \quad 4.53$

Versuchsreihe Tabelle 7 , Rohr II $\left(652^{\circ}\right)$.

$\begin{array}{llllllll}\text { Zeit } \ldots \ldots \ldots \ldots \ldots & 0.227 & 0.346 & 0.467 & 0.703 & 1.97 & 2.67\end{array}$

$k$ nach Boden-

$\begin{array}{llllll}\text { stein.......87.3 } & 71 \cdot 7 & 77 \cdot 1 & 77 \cdot 0 & 67 \cdot 5 & 94.8\end{array}$

$\frac{4 k p^{2}}{R^{2} T^{2}}$ nach

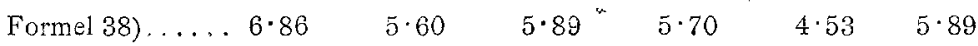

Versuchsreihe Tabelle 8 , Rohr II $\left(670^{\circ}\right)$.

$\begin{array}{lllllll}\text { Zeit } \ldots \ldots \ldots \ldots & 0.197 & 0.449 & 0.715 & 1.134 & 1.490 & 2.398\end{array}$

$k$ nach Boden-

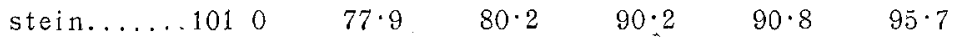

$\frac{4 k p^{2}}{R^{2} T^{2}}$ nach

$\begin{array}{lllllll}\text { Forme1 38) } \ldots & 8.17 & 6.04 & 5.99 & 6.36 & 6.33 & 6.12\end{array}$

Versuchsreihe Tabelle 9 , Rohr II $\left(689^{\circ}\right)$.

$\begin{array}{llllll}\text { Zeit ......... } 0.178 & 0.336 & 0.479 & 0.623 & 0.651 & 1.009\end{array}$

$k$ nach Boden-

$\begin{array}{llllll}\text { stein.....168 } & 163 & 168 & 163 & 161 & 154\end{array}$

$\frac{4 k p^{2}}{R^{2} T^{2}}$ nach

$\begin{array}{llllll}\text { Formel 38) } \ldots 1 \check{2} \cdot 7 & 11 \cdot 7 & 11 \cdot 7 & 11 \cdot 1 & 10 \cdot 9 & 9 \cdot 93\end{array}$ 
Da die Mittelwerte der Constanten und die mittieren Fehler der einzelnen Werte durch die erheblichen zufälligen Versuchsfehler stark beeinflusst werden, ist die graphische Darstellung der Resultate das geeignetste Mittel zur Prüfung, $a b$ die Berechnung nach Bodenstein oder nach Formel 38) bessere Resultate gibt.

In der beiliegenden Darstellung sind als Abscissen die umgesetzten Mengen gewählt, um einen für alle Versuchsreihen einheitlichen Maßstab zu haben. (Es sei hiebei auf eine in Tabelle 6 auftretende Störung aufmerksam gemacht. Der vorletzte eingerahmte Punkt ist zeitlich der letzte. Der gefundene Umsatz war zur Zeit 1.720 .452 , zur Zeit 1.73 $0 \cdot 433$.

Als Ordinaten sind die berechneten Constanten in willkürlichen, für jede Versuchsreihe und Berechnung verschiedenen Maßstäben aufgetragen, um ein übersichtliches Bild zu bekommen.

Die Curven sollen der Abscissenaxe parallele Gerade sein. Dagegen verstoßen zunächst bei beiden Berechnungen Tabelle 1 und je der erste Wert in Tabelle 7 und 8. Es handelt sich da durchwegs um geringe Umsätze, also um Bedingungen, wo ein erheblicher Unterschied zwischen den beiden Berechnungen nicht auftreten kann.

Ferner zeigen beide der Tabelle 9 entsprechenden Curven einen absteigenden Gang, und zwar die der Gleichung 38) entsprechende Curve stärker als die Bodenstein'sche. Diese Versuchsreihe ist bei einer Temperatur ausgeführt, die nur 10 bis $20^{\circ}$ unter der Explosionstemperatur liegt. Unter diesen Umständen ist ein abnormer Reactionsverlauf nicht unwahrscheinlich.

Die Curven der Tabellen 2, 10,6,7 und 8 (die beiden letzteren mit Hinweglassung der ersten Beobachtung) zeigen deutlich die Überlegenheit der Formel 38). Denn die Curven nach Bodenstein haben unverkennbar einen aufsteigenden Gang (insbesondere bei Tabelle 2 und 8), welcher bei derBerechnung nach Formel 38) verschwindet. Die nach dieser Formel berechneten Constanten fallen bei Tabelle 10, 2, 7 und 8 (bei letzteren drei nach Hinweglassung je eines Wertes) ziemlich 
befriedigend aus. Nur Tabelle 6 gibt auch nach Gleichung 38) schlechte Constanten.

Die Tabelle $4 A$ ist in die graphische Darstellung nicht aufgenommen, da der Umsatz bis $71.4 \%$ anstieg. Die Überlegenheit der Formel 38) ist bei dieser Versuchsreihe aus den Zahlen unmittelbar ersichtlich.

§. 101. Ähnlich gestaltet sich die Sache bei den Versuchen mit äquivalenten Mengen im Rohre I.

Versuchsreine Tabelle 1, Rohr I $\left(482^{\circ}\right)$.

$\begin{array}{llllll}\text { Zeit } \ldots \ldots \ldots .41 \cdot 3 & 67 \cdot 1 & 95 \cdot 7 & 124 \cdot 3 & 156 \cdot 5 & 189 \cdot 5\end{array}$

$k$ nach Boden-

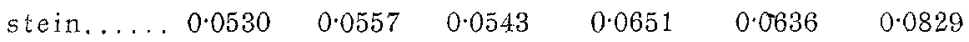

$\frac{4 k p^{2}}{R^{2} T^{2}}$ nach

Forme1 38). $0.00666 \quad 0.00696 \quad 0.00676 \quad 0.00798: 0.00776 \quad 0.01005$

Der höchste Umsatz war nur $17 \%$. Daher kann ein erheblicher Unterschied zwischen beiden Berechnungen nicht auftreten.

Versuchsreihe Tabelle 2 , Rohr I $\left(509^{\circ}\right)$.

\begin{tabular}{|c|c|c|c|c|}
\hline Zeit & $\ldots \ldots \ldots \ldots \ldots 17 \cdot 39$ & $37 \cdot 19$ & $121 \cdot 70$ & $192 \cdot 00$ \\
\hline$k$ nach & Bodenstein.... 0.257 & $0 \cdot 216$ & $0 \cdot 250$ & 0.398 \\
\hline $4 k p^{2}$ & nach Formel 38) ... 0.0310 & 0.0243 & 0.0288 & 0.0374 \\
\hline
\end{tabular}

Ich habe aus dieser ziemlich unregelmäßig verlaufenen Versuchsreihe nur wenige Werte berechnet. Sie genügen, um zu zeigen, dass die Schwankungen der Constanten durch die Berechnung nach Formel 38) vermindert werden.

\section{Versuchsreihe Tabelle 6, Rohr I $\left(638^{\circ}\right)$.}

\begin{tabular}{|c|c|c|c|c|c|c|}
\hline Zeit ...... 0.77 & 1.08 & $1 \cdot 67$ & $2 \cdot 15$ & $=2 \cdot 98$ & $3 \cdot 47$ & $3 \cdot 48$ \\
\hline $\begin{array}{l}\text { nach Boden- } \\
\text { stein.....31.1 }\end{array}$ & $3,3 \cdot 0$ & $35 \cdot 4$ & $51 \cdot 5$ & $43 \cdot 9$ & $39 \cdot 6$ & $38 \cdot 4$ \\
\hline $4 k p^{2}$ & & & & & & \\
\hline $2 T^{2}$ & & & & & & \\
\hline Formel 38$) \ldots 2 \cdot 57$ & $2 \cdot 70$ & $2 \cdot 78$ & $3 \cdot 79$ & $3 \cdot 17$ & $2 \cdot 83$ & $2 \cdot$ \\
\hline
\end{tabular}


Auch hier gibt Formel 38) constantere Werte. Unregelmäßiger verläuft die folgende Versuchsreihe:

Versuchsreihe Tabelle $4 A$, Rohr I $\left(572^{\circ}\right)$.

\begin{tabular}{|c|c|c|c|c|c|c|}
\hline Zeit . . . . . 1.96 & $5 \cdot 45$ & $14 \cdot 93$ & $22 \cdot 72$ & $42 \cdot 35$ & $47 \cdot 82$ & $58 \cdot 81$ \\
\hline $\begin{array}{l}\text { nach Boden- } \\
\text { stein....2. }\end{array}$ & $2 \cdot 27$ & $2 \cdot 48$ & $2 \cdot 07$ & $2 \cdot 42$ & $2 \cdot 15$ & $4 \cdot 09^{1}$ \\
\hline $4 k p^{2}$ & & & & & & \\
\hline$T^{2}$ & & & & & & \\
\hline ormel 38). $.0 \cdot 280$ & $\cdot 222$ & 0.226 & $0 \cdot 186$ & $0 \cdot 200$ & 0.177 & 0.304 \\
\hline
\end{tabular}

Es ist unverkennbar, dass die Versuche im Rohre I größeren Störungen unterworfen waren als im Rohre II. Rohr I war ein weites U-Rohr, Rohr II ein enges Schlangenrohr. Es llegt daher nahe, zu vermuthen, dass die Unregelmäßigkeiten der Versuche zum großen Theile durch unvollständige Ableitung der Reactionswärme und durch Strömungen in der Röhre hervorgerufen werden. Letztere mischen Theile des Gases, in denen die Reaction bereits weiter fortgeschritten ist, mit solchen, in denen der Umsatz erst einen geringen Betrag erreicht hat, und bewirken daher eine Abweichung von der bei der Rechnung gemachten Voraussetzung, dass die auftretenden Concentrationsänderungen nur durch die Reaction hervorgerufen werden.

Beide Störungen müssen im Rohre I wirksamer sein als im Rohre II.

§. 102. Bei den Versuchen mit nicht äquivalenten Mengen gibt die Formel 37) keine besseren Resultate als die Bodenstein'sche Berechnung. Die von Bodenste in berechneten Constanten dieser Versuche zeigen meistens einen deutlichen Gang, indem sie mit steigender Zeit fallen. Dieser Gang wird noch ausgesprochener bei Anwendung der Formel 37). Es ist kaum zu bezweifeln, dass bei diesen Versuchen besondere Störungen mitwirkten, welche bei den Versuchen mit äquivalenten Mengen keine Rolle spielten. Bodenstein hebt selbst verschiedene auffällige Erscheinungen bei diesen Versuchen hervor. ${ }^{2}$

1 In der Abhandlung steht irrthümlich $3 \cdot 24$.

2 A. a. O., S. 683. 
Für das Gemisch $4 \mathrm{H}_{2}+\mathrm{O}_{2}$ ergibt sich die Formel

$$
\begin{array}{r}
\frac{4 k p^{2}}{R^{2} T^{2}}=\frac{1}{t}\left\{-\frac{4 \cdot 5 x}{2-x}+34 \cdot 538775 \log \left(1-\frac{x}{2}\right)-\right. \\
-36 \cdot 84136 \log (1-x)\},
\end{array}
$$

für $2 \mathrm{H}_{2}+2 \mathrm{O}_{2}$

$$
\begin{aligned}
\frac{4 k p^{2}}{R^{2} T^{2}}=\frac{1}{t}\left\{\frac{9 x}{1-x}+6 \cdot 907755\right. & \log (1-x)- \\
& \left.-9 \cdot 21034 \log \left(1-\frac{x}{2}\right)\right\} .
\end{aligned}
$$

Im folgenden sind die nach diesen Formeln berechneten Constanten mit den von Bodenstein berechneten zusammengestellt.

$$
\text { Tabelle IV } B \text {, Rohr I. }
$$

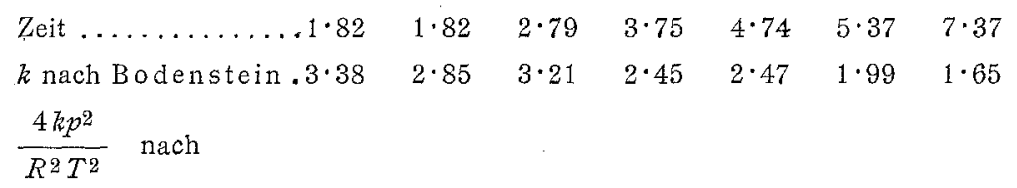

Formel 39) $\ldots \ldots \ldots 0.337 \quad 0.282 \quad 0.311 \quad 0.242 \quad 0.242 \quad 0.198 \quad 0.161$

\section{Tabelle IV $B$, Rohr II.}

$\begin{array}{llllllllll}\text { Zeit .......... } & 1.14 & 1.14 & 1.76 & 2.37 & 2.99 & 3.38 & 4.77 & 6.06 & 12.50\end{array}$ $k$ nach Boden$\begin{array}{lllllllll}\text { stein } \ldots \ldots \ldots 15 \cdot 7 & 15 \cdot 9 & 15 \cdot 6 & 15 \cdot 6 & 14 \cdot 2 & 14 \cdot 3 & 12 \cdot 7 & 12 \cdot 0 & 12 \cdot 0\end{array}$ $\frac{4 k p^{2}}{R^{2} T^{2}}$ nach

$\begin{array}{llllllllll}\text { Formel } 40) \ldots \ldots & 1.52 & 1.53 & 1.47 & 1.45 & 1.30 & 1.30 & 1.13 & 1.05 & 0.98\end{array}$

$$
\text { Tabelle IV } C \text {, Rohr } 1 .
$$

$\begin{array}{lll}\text { Zeit } \ldots \ldots \ldots \ldots \ldots \ldots \ldots .06 & 1 \cdot 30 & 7 \cdot 23 \\ k \text { nach Bodenstein...... } 3 \cdot 16 & 2 \cdot 44 & 1 \cdot 50 \\ \left.\frac{4 k p^{2}}{R^{2} T^{2}} \text { nach Formel } 40\right) \ldots \ldots .299 & 0 \cdot 243 & 0 \cdot 147\end{array}$


Tabelle IV $C$, Rohr II.

$\begin{array}{llllllllllll}\text { Zeit....... } & 0.43 & 0.44 & 0.53 & 0.67 & 0.85 & 1.02 & 1.30 & 1.56 & 1.92 & 2.13 & 3.05\end{array}$

$k$ nach Boden-

$\begin{array}{lllllllllll}\text { stein } \ldots .28 \cdot 0 & 28.5 & 26.6 & 27.0 & 26.9 & 27.1 & 25 \cdot 1 & 25.4 & 24.4 & 25.9 & 25.8\end{array}$

$\frac{4 k p^{2}}{R^{2} T^{2}}$ nach

$\begin{array}{llllllllllll}\text { Forme1 39) } & 2.73 & 2.78 & 2.56 & 2.55 & 2.56 & 2.55 & 2.35 & 2.34 & 2.23 & 2.33 & 2.18\end{array}$

§. 103. Im ganzen glaube ich sagen zu können, dass die Neuberechnung den Wert der Versuche Bodensteins mit Rohr II nur noch deutlicher hervortreten lässt und dass sie, indem sie einen Unterschied zwischen den Röhren I und II erkennen lässt, zur Aufklärung der Störungen einen Beitrag liefert.

\section{Zusammenfassung.}

1. Es wird eine allgemeine Form der Gleichungen für die Reactionsgeschwindigkeiten in homogenen Systemen bei constantem Volum entwickelt, welche alle Arten von Reactionen (Nebenwirkungen, Gegenwirkungen, Folgewirkungen, katalytische Wirkungen) umfasst.

2. Mit Hilfe dieser allgemeinen Darstellung der Reactionsgeschwindigkeiten wird untersucht, wie die Reactionsgleichungen beschaffen sein müssen und welche Bedingungen außerdem erfüllt sein müssen, damit

a) die Geschwindigkeiten zweier nebeneinander verlaufender Reactionenen in einem von der Zeit unabhängigen Verhältnisse stehen;

B) die Concentration eines Stoffes unverändert bleibt, welcher durch nebeneinander verlaufende Reactionen theils gebildet, theils verbraucht wird;

$\gamma)$ die Mengenänderungen zweier Stoffe in einem von der Zeit unabhängigen Verhältnisse stehen. Hievon wird auf das constante Umwandlungsverhältnis bei der Cinchoninumlagerung (Skraup) Anwendung gemacht. 
3. Es wird gezeigt, wie die Gleichungen für die Reactionsgeschwindigkeiten bei wechselndem Volum und insbesondere bei Gasen unter constantem Drucke aufzustellen sind. Die erhaltenen Formeln werden zur Berechnung der Versuche von Bodenstein über die Bildung von Wasserdampf aus Knallgas verwendet. 